\title{
Size Dependence of Structures and Properties of Magnetic Materials
}

\author{
Q. Jiang ${ }^{*}$ and X.Y. Lang
} Key Laboratory of Automobile Materials (Jilin University), Ministry of Education, and Department of Materials Science
and Engineering, Jilin University, Changchun 130025, China

\begin{abstract}
Due to the involvement of high portion of atomic coordination imperfection in surfaces and interfaces, the properties of magnetic nanocrystals dramatically differ from that of the corresponding bulk counterparts, which have led to a surge in both experimental and theoretical investigations in the past decades. This review summarizes the studies for these size-dependent magnetic properties, and additional thermal or phase stabilities, and mechanical properties. To interpret the above phenomena, a thermodynamic model for the size dependence and interface dependences of these properties is described, which is based on Lindemann's criterion for melting, Mott's expression for the vibrational melting entropy, and Shi's model for the size-dependent melting temperature. The model without any adjustable parameter is confirmed by a large number of experimental results, and helps us to understand the structures and properties of the magnetic nanocrystals. Moreover, other theoretical works on the above properties are also mentioned and commented.
\end{abstract}

\section{INTRODUCTION}

In modern life, magnetic materials can be found in a variety of applications that range from microelectronic devices to motors and power distribution system, computer technique, magnetic high-density recording, etc. At the emerging nanotechnology era, low-dimensional magnetic systems like thin films [1], multilayers [2], nanorods and nanoparticles, as well as interacting magnetic nanostructures [3], have attracted tremendous interest and opportunities for industrial applications [4]. Due to the current progress in nanotechnology, individual magnetic nanocrystals can be fabricated, which in turn provides opportunities to study basic properties of nanocrystals via various experimental techniques, theoretical methods and compute simulations.

Commonly, the magnetic nanocrystals may be elements, compounds, alloys, and even organic molecules. From the fundamental point of view, magnetic nanocrystals with different dimensions bridge the gap between isolated atoms and their bulk counterparts in chemical, physical, mechanical properties while the interatomic interaction causes a solid to differ completely from an isolated atom in performance [5]. Compared with the bulk counterparts, nanocrystals involve high surface/volume ratio, which leads to the intrinsic size dependences in the mechanical strength, the thermal and phase stabilities, as well as the magnetic properties [6], for the example of the appearance of superparamagnetism. Because of the breaking of lattice symmetry or surface/interface bond changes, thermal stability of phases, the site-specific surface anisotropy, the surface spin disorder and the weakened magnetic exchange coupling vary. These variable properties can functionalize sensors or switches, and apply in device areas of high-density perpendicular recording, colour imaging, ferrofluids, ultrahigh frequency $(0.3-3 \mathrm{GHz})$ devices, magnetic refrigeration and drug carries for site-

*Address correspondence to this author at the Key Laboratory of Automobile Materials (Jilin University), Ministry of Education, and Department of Materials Science and Engineering, Jilin University, Changchun 130025, China; Fax: +86 431 5095876; E-mail: jiangq@jlu.edu.cn specific drug delivery. The coupling FM/AFM bilayers can be utilized in permanent magnets, magnetic recording media, and domain stabilizers in recording heads based on anisotropic magnetoresistance.

On the other hand, nanostructure magnetic materials consisting of densely parked grains often reveal different properties from isolated nanocrystals since the surface skins now are substituted by the interface ones. For instance, isolated nanoparticles above the onset of superparamagnetism are magnetically hard with large coercivity while nanostructured magnets are often magnetically soft due to the interaction among grains [7].

Over the past decades, an overwhelming contribution has been made to develop nanotechnology by the advent of methods, such as atomic imaging and manipulating, nanocrystalline synthesizing, functioning, and characterizing as well as structural patterning for device fabrication. However, insight into the underlying mechanisms and factors, which dominate the general trend of the tunability, remains in its infancy. Although a number of models have been proposed to interpret the size dependences, they, respectively, often correspond to a part of phenomena. A unified model dealing with all related phenomena is thus highly desirable. This unification certainly gives rise to an understanding of sizedependent properties of magnetic nanocrystals.

The main objective of this contribution is to present a review with special attentions to the thermal and phase stabilities as well as the statical magnetic properties of FM, AFM nanocrystals and FM/AFM bilayer systems. On the basis of these phenomena, a thermodynamic approach is proposed to interpret the observed experimental results and the underlying mechanism for size-dependent properties of nanocrystals. Deeper and consistent insight into the mechanism behind the observations and finding factors dominate general trends of size-induced property change, which are of fundamental importance for advancing technological applications. In section 2, main interest is on the thermal stability and the mechanical properties of magnetic nanocrystals. This 
section summaries several theoretical models to illustrate the physical nature of thermal stability of nanocrystals with special emphasis on lattice thermal instability model and its extending on the size dependences of mechanical properties of hardness and yield strength. Sections 3 and 4 introduce the size-dependent magnetic properties of ferromagnetic (FM) and antiferromagnetic (AFM) nanocrystals in experiments, such as size-induced magnetization, anisotropy, coercivity and superparamagnetism, where the lattice thermal instability model is also introduced to illustrate the underlying mechanisms. Section 5 conveys the theoretical and experimental investigations of FM/AFM bilayers, in particular the size effects of exchange bias field and its thermal stability. Finally, concluding remarks are presented in section 6 with suggestions for future directions in extending the developed knowledge and the associated approaches.

\section{THERMAL STABILITY AND MECHANICAL PROPERTIES OF MAGNETIC NANOCRYSTALS}

\subsection{Thermal Stability of Nanocrystalline Magnetic Mate- rials}

Thermal stability of nanocrystals is an important quantity for designing of devices and governing materials for practical applications, which is characterized by melting temperature $T_{\mathrm{m}}(D)$ where $D$ denotes the thickness of thin films, the diameter of nanoparticles or nanorods, respectively. Over the past decades, following Takagi's experimental pioneering work in 1954 [8], which demonstrated $T_{\mathrm{m}}(D)<T_{\mathrm{m}}(\infty)$ where $\infty$ denotes the bulk size, thermal stabilities of metallic [9], organic [10,11], semiconductor [12] nanocrystals have been intensively studied experimentally and theoretically. It has been revealed that the crystalline lattice spacing in nanocrystals can be altered by surface relaxation [13], stress [14], or strain. Similarly, the restricted geometry of such systems can exclude long-wavelength phonons and emphasize surface modes, leading to unusual phonon spectra. Therefore, nanocrystals can exhibit not only melting point depression, but also superheating, depending on their surrounding environment [15].

Magnetic nanocrystals consisting of metallic $\mathrm{Fe}, \mathrm{Co}, \mathrm{Ni}$ and their alloys, soft and hard magnets, ferrofluids, and nanocomposites have attracted great interest in preparations and characterizations. Numerous physical and chemical methods have been employed to produce these magnetic nanocrystals, including molecular beam epitaxy, pulsed laser deposition, chemical vapor deposition, electrodeposition, flash evaporation, temperature-directed growth, and neutral organometallic precursor decomposition. Chemical methods, in particular, solution routes, are widely used for the fabrication of nanoparticles and nanocomposites [16]. Their thermal properties have also been studied by computer simulations (Monte Carlo [17] and molecular dynamics [18-21]) and experimental techniques, such as transition electron microscopy, Mössbauer spectroscopy, small and wide angle X-ray diffraction as well as positron annihilation [6]. It has been found that at temperature $T<T_{\mathrm{m}}$, the lattice vibrations of $\mathrm{Fe}$ $[22,23]$ and Co [24] nanocrystals increase, which drops their corresponding Debye temperatures $\Theta_{\mathrm{D}}(D)$. In terms of Lin- demann's criterion, the root of atomic mean-square displacement $\sigma$ of crystals increases with increasing $T$. When $T$ approaches $T_{\mathrm{m}}, \sigma$ reaches a fraction of atomic diameter $h$ and melting occurs. Because $\sigma$ increases with decreasing $D$, the needed thermal energy to promote nanocrystals to melt decreases as $D$ is reduced, and hence $T_{\mathrm{m}}(D)$ decreases [18-21].

Thermal stability of nanocrystals is also responsible for other thermally activated behaviors such as atomic diffusion $[25,26]$ and crystal growth $[27,28]$, which in turn influences the magnetic properties of nanocrystals because they depend not only on $D$, but on crystalline structures and presence of lattice defects [29-31].

\subsubsection{Theoretical Models for $T_{m}(D)$ Functions}

Classically, the melting of nanocrystals has been described by three kinds of models [32]: homogeneous melting model without a liquid skin [33,34], liquid-skin melting model $[35,36]$ and liquid nucleation and growth model with an unstable liquid skin $[37,38]$.

The first and the earliest thermodynamic model, for $T_{\mathrm{m}}(D)$ function, considers the equilibrium between the entire solid and liquid where the melting proceeds throughout the solid simultaneously [34],

$$
T_{\mathrm{m}}(D) / T_{\mathrm{m}}(\infty)=1-V_{\mathrm{m}}\left[\gamma_{\mathrm{sv}}-\gamma_{\mathrm{lv}}\left(\rho_{\mathrm{s}} / \rho_{\mathrm{l}}\right)^{2 / 3}\right] /\left(D H_{\mathrm{m}}\right)
$$

where $V_{\mathrm{m}}$ denotes the molar volume of solids, $\gamma$ shows the surface/interface energy, $\rho$ is the mass density, $H_{\mathrm{m}}$ is the melting enthalpy, and subscripts $\mathrm{s}, 1, \mathrm{v}$ denote solid, liquid, vapor, respectively. For the most cubic metals $[39,40], \gamma_{\mathrm{sv}}-$ $\gamma_{\mathrm{lv}} \approx \gamma_{\mathrm{sl}}$. If $\rho_{\mathrm{s}} \approx \rho_{\mathrm{l}}$ then $\left(\rho_{\mathrm{s}} / \rho_{\mathrm{l}}\right)^{2 / 3} \approx 1$ and thus Eq. (1) can be rewritten as

$$
T_{\mathrm{m}}(D) / T_{\mathrm{m}}(\infty) \approx 1-2 V_{\mathrm{m}} \gamma_{\mathrm{sl}} /\left(D H_{\mathrm{m}}\right)
$$

Equation 2 is identical to the Gibbs-Thomson equation $[10,41] T_{\mathrm{m}}(D) / T_{\mathrm{m}}(\infty)=1-\left(2 / D_{1}+2 / D_{2}\right) V_{\mathrm{m}} \gamma_{\mathrm{sl}} / H_{\mathrm{m}}$ with $D_{1}$ and $D_{2}$ as the principal diameters of curvature of the crystal being in equilibrium on the interface with the liquid at $T_{\mathrm{m}}\left(D_{1}, D_{2}\right)$ under condition that for a spherical particle, $1 / D_{1}$ $=1 / D_{2}=1 / D$.

The liquid-skin melting model assumes that a liquid layer with a fitting thickness of $\beta$ is in equilibrium at the solid surface and surface melts before the melting of the solid core $[35,36]$,

$$
\begin{aligned}
& T_{\mathrm{m}}(D) / T_{\mathrm{m}}(\infty)=1-2 V_{\mathrm{m}}\left[\gamma_{\mathrm{sl}} /(1-\beta / D)\right. \\
& \left.-\gamma_{\mathrm{lv}}\left(1-\rho_{\mathrm{s}} / \rho_{\mathrm{l}}\right)\right] /\left(D H_{\mathrm{m}}\right)
\end{aligned}
$$

The liquid nucleation and growth model suggests that melting starts by the nucleation of a liquid layer at the surface and moves into the solid as a slow process with a definite activation energy [37,38],

$$
\begin{aligned}
& T_{\mathrm{m}}(D) / T_{\mathrm{m}}(\infty)=1-\left[3\left(V_{\mathrm{ms}}+V_{m l}\right)\left(\gamma_{\mathrm{Ms}}-\gamma_{\mathrm{Ml}}\right)\right. \\
& / 2 D-\Delta U] / H_{\mathrm{m}}
\end{aligned}
$$

where subscript $\mathrm{M}$ denotes the solid matrix, $\Delta U$ shows the energy density difference between crystal and liquid. If $\Delta U$ 
is negligible then, depending on the sign of $\gamma_{\mathrm{Ms}}-\gamma_{\mathrm{Ml}}, T_{\mathrm{m}}(D)$ can either be higher or lower than $T_{\mathrm{m}}(\infty)$, which is closely related to the nature of the interface. Generally, $\gamma_{\mathrm{Ml}}-\gamma_{\mathrm{Ms}}=$ $\gamma_{\mathrm{s} 1} \cos \theta$, where $\theta$ is the contact angle between a particle and the matrix ranged from $0^{\circ}$ to $180^{\circ}$. For a particle wetted by the matrix, $0^{\circ} \leq \theta<90^{\circ}$ and $0<\gamma_{\mathrm{Ml}}-\gamma_{\mathrm{Ms}} \leq \gamma_{\mathrm{sl}}$ where the matrix/particle interface should be coherent or semi-coherent. Consequently, superheating happens and $T_{\mathrm{m}}(D)$ increases with decreasing $D$. For a nanocrystal with $\theta \geq 90^{\circ}, \gamma_{\mathrm{Ml}}-\gamma_{\mathrm{Ms}} \leq$ 0 and undercooling occurs. Here it should be noted that the mechanisms of the first and the second models describe the melting of a flat surface or larger nanoparticles.

$T_{\mathrm{m}}(D)$ functions in all three models show that they vary inversely with $D: T_{\mathrm{m}}(D) / T_{\mathrm{m}}(\infty)=1-4 C /\left(\rho_{\mathrm{s}} H_{\mathrm{m}} D\right)$ where $C$ value differs only slightly among the models. Ignoring difference of $\rho_{\mathrm{l}}$ and $\rho_{\mathrm{s}}, C \approx \gamma_{\mathrm{sv}}-\gamma_{\mathrm{lv}}, C \approx \gamma_{\mathrm{sl}}$, and $C=1.5\left(\gamma_{\mathrm{sv}}-\gamma_{\mathrm{lv}}\right)$ $\sim \gamma_{\mathrm{sl}}$ in these models [42].

Recently, Sun [43] established relationship of $T_{\mathrm{m}}(D)$ function directly to the coordination number $(C N)$ imperfection effect on atomic cohesive energy $\left(E_{\mathrm{c}}=z N_{\mathrm{a}} \varepsilon / 2\right.$ being the sum of bond energy $\varepsilon$ over all coordinates of a specific atom with the coordination $z$ and the Avogadro number $N_{\mathrm{a}}$ ) of the lower coordinated atoms near the surface. The $\mathrm{CN}$ imperfection causes the remaining bonds of the lowercoordinated atoms to contract with increase of magnitude of $\varepsilon$, i.e., bond-order-length-strength (BOLS) correlation [44], which contributes to $E_{\mathrm{c}}$, and hence to the Gibbs free energy that determines the thermodynamic behavior of a system. The thermal energy required to loosen the bonds of the specific atom is a portion of $E_{\mathrm{c}}$. Thus, $T_{\mathrm{m}}(D) \propto E_{\mathrm{c}}(D)$, which leads to [45],

$$
T_{m}(D) / T_{m}(\infty)=1+\sum_{i \leq 3} n_{i}\left(z_{s v} q_{s}^{-w}-1\right)
$$

where $q_{\mathrm{s}}=h_{\mathrm{s}} / h=2 /\left\{1+\exp \left[\left(12-z_{\mathrm{s}}\right) /\left(8 z_{\mathrm{s}}\right)\right]\right\}$ shows a $C N$ dependent reduction of $h, z_{\mathrm{sv}}=z_{\mathrm{s}} / z_{\mathrm{v}}$ with subscripts $s$ and $v$ denoting surface and bulk, respectively, and $n_{\mathrm{i}}=(3-d) h q_{\mathrm{s}} / D$ is the portion of atoms in the $i$-th atomic layer from the surface compared to the total atom number of the solid $n_{\mathrm{t}}$, and the dimension $d=0,1,2$ corresponds to nanoparticles, nanorods, and thin films, respectively. The power index $w$ is an indicator for the bond nature of a specific material. $w=1$ for elemental metals, $w=4$ for alloys or compounds, and $w=$ 2.56 and 4.88 for $\mathrm{C}$ and $\mathrm{Si}$, respectively [5]. The model indicates that $T_{\mathrm{m}}$ change arises from the change of atomic $E_{\mathrm{c}}$ in the superficial skins.

\subsubsection{Atomic Vibrational Instability}

The termination of the lattice periodicity in the surface normally leads to the $C N$ reduction of surface atoms and the creation of a surface potential barrier [5], which change surface physical quantities $\left[Q_{\mathrm{s}}(D)\right]$ of nanocrystals being different from the internal counterparts $\left[Q_{\mathrm{v}}(D)\right]$. Based on meanfield approximation, a $Q(D)$ function can be represented as $[46,47]$,

$$
Q\left(D=Q_{\mathrm{v}}(D)+\left[Q_{\mathrm{s}}(D)-Q_{\mathrm{v}}(D)\right] n_{\mathrm{s}} / n_{\mathrm{t}}\right.
$$

where $n_{s} / n_{\mathrm{t}} \propto 1 / D$ is the ratio of the number of surface atoms $\left(n_{\mathrm{s}}\right)$ to the total atom number $\left(n_{\mathrm{t}}\right)$ of nanocrystals.

If the cooperative coupling between the surface region and the interior region is phenomenologically considered by taking the variation of $Q(D)$ to be dependent on the value of $Q(D)$ itself, a change in $Q$ can give rise to $Q(x+d x)-Q(x)=$ $(\alpha-1) Q(x) d x$, which is achieved by assuming that $Q_{\mathrm{s}} / Q_{\mathrm{v}}$ are size-independent, and $\alpha=Q_{\mathrm{s}}(D) / Q_{\mathrm{v}}(D) \approx Q_{\mathrm{s}}(\infty) / Q_{\mathrm{v}}(\infty), x=$ $n_{\mathrm{s}} / n_{\mathrm{v}}=D_{0} /\left(D-D_{0}\right)$ with $D_{0}$ denoting a critical size at which all the atoms or molecules of low-dimensional nanocrystals are located on their surfaces. In terms of the definition of $D_{0}$ and the bulk boundary condition, two asymptotic limits should be satisfied, namely $Q(D) / Q(\infty) \rightarrow 0$, when $D \rightarrow D_{0}$, and $Q(D) / Q(\infty) \rightarrow 1$, when $D \rightarrow \infty$. Associated with the conditions and integrating the above equation, it reads,

$$
Q(D) / Q(\infty)=\exp \left[(\alpha-1) n_{\mathrm{s}} / n_{\mathrm{v}}\right]=\exp \left[(\alpha-1) /\left(D / D_{0}-1\right)\right]
$$

$D_{0}$ is a function of $d$. In general, the dimension can be fractal [48]. For $d=0, D_{0}=6 h$ since $4 \pi h\left(D_{0} / 2\right)^{2}=$ $4 \pi\left(D_{0} / 2\right)^{3} / 3$; For $d=1, D_{0}=4 h$ since $2 \pi h\left(D_{0} / 2\right)=\pi\left(D_{0} / 2\right)^{2}$; For $d=2, D_{0}=2 h$ since $2 h=2\left(D_{0} / 2\right)$. In short,

$$
D_{0}=2(3-d) h
$$

Letting $Q(D)$ being the mean-square displacement of nanocrystals $\sigma^{2}(D)$, one obtains the extension of Eq. (7) from Lindemann's melting criterion [49], namely, $\sigma^{2}(D) / \sigma^{2}(\infty)=$ $\exp \left[(\alpha-1) /\left(D / D_{0}-1\right)\right]$. Since $T_{\mathrm{m}}(\infty)$ are usually higher than the bulk Debye temperature $\Theta_{\mathrm{D}}(\infty)$, the high temperature approximation can be utilized $[46,50], \sigma^{2}(D, T)=F(D) T$ where $F(D)$ is the $T$-independent but size-dependent parameter with $T$ being the absolute temperature. Thus, $\sigma^{2}(D, T) / \sigma^{2}(\infty, T)=$ $F(D) / F(\infty)$. When $T=T_{\mathrm{m}}, F(D) / F(\infty)=\left\{\sigma^{2}\left[D, T_{\mathrm{m}}(D)\right] / h^{2}\right\} /$ $\left\{\sigma^{2}\left[\infty, T_{\mathrm{m}}(\infty)\right] / h^{2}\right\}\left[T_{\mathrm{m}}(\infty) / T_{\mathrm{m}}(D)\right]=T_{\mathrm{m}}(\infty) / T_{\mathrm{m}}(D)$ in terms of Lindemann's criterion. Here $h-h(D) \approx 0$ is assumed since [ $h$ $h(D)] / h$ is negligible when $D>20 \mathrm{~nm}$ and $[h-h(D)] / h=0.1-$ $2.5 \%$ when $D<20 \mathrm{~nm}$. Note also that $\Theta_{\mathrm{D}}(\infty) \propto$ $\left[T_{\mathrm{m}}(\infty) /\left(M V_{\mathrm{m}}(\infty)^{2 / 3}\right)\right]^{1 / 2}[51]$ where $M$ is the molecular weight and $V_{\mathrm{m}}(\infty)$ is the bulk molar volume. $\Theta_{\mathrm{D}}(D)$ function can be obtained as a generalization of above relationship, i.e., $\Theta_{\mathrm{D}}^{2}(D) / \Theta_{\mathrm{D}}^{2}(\infty)=T_{\mathrm{m}}(D) / T_{\mathrm{m}}(\infty)$. In summary [46,50],

$$
\begin{aligned}
& T_{\mathrm{m}}(D) / T_{\mathrm{m}}(\infty)=\sigma^{2}(\infty) / \sigma^{2}(D)=\Theta_{\mathrm{D}}^{2}(D) / \Theta_{\mathrm{D}}{ }^{2}(\infty)= \\
& \exp \left[-(\alpha-1) /\left(D / D_{0}-1\right)\right]
\end{aligned}
$$

In Eq. (9), the smallest size of crystals is assumed to be $2 D_{0}$ where a half of atoms of a crystal is located on the surface with $n_{\mathrm{s}} / n_{\mathrm{v}}=1$. Thus it takes the form $T_{\mathrm{m}}\left(2 D_{0}\right)=$ $T_{\mathrm{m}}(\infty) \exp (1-\alpha)$ where both crystal and liquid have almost the same short range order and their structure difference is little and as a result, melting disappears [52].

By considering the vibrational entropy of melting of nanocrystals $S_{\mathrm{vib}}(D)$ deduced from Mott's expression for $S_{\text {vib }}(\infty)$ [53] is written as [11],

$$
S_{\mathrm{vib}}(D)=S_{\mathrm{vib}}(\infty)-(3 R / 2)(\alpha-1) /\left(D / D_{0}-1\right)
$$


where $R$ is the ideal gas constant. For a free-standing or a quasi-free-standing nanocrystal, $S_{\mathrm{m}}\left(2 D_{0}\right) \approx S_{\text {vib }}\left(2 D_{0}\right)=0$ is assumed. Thus, the value of $\alpha_{s}$ can be determined as [11],

$$
\alpha_{\mathrm{s}}=1+2 S_{\mathrm{vib}}(\infty) /(3 R)
$$

As a rule, melting entropy $S_{\mathrm{m}}(\infty)$ consists, at least, of positional $S_{\text {pos }}(\infty)$, vibrational $S_{\text {vib }}(\infty)$ and electric $S_{\text {el }}(\infty)$ [54], $S_{\mathrm{m}}(\infty)=S_{\mathrm{vib}}(\infty)+S_{\text {pos }}(\infty)+S_{\text {el }}(\infty)$. The idea of $S_{\text {pos }}(\infty)$ of melting arises in connection with the positional disorder as a substance undergoes the melting transition. The number of particle species naturally plays a primary role involved in the disordering process. In the case of simple solids, only two particle species are present: the atoms of the given substance and vacancies [54]. In this case, $S_{\text {pos }}(\infty)$ is given by [54], $S_{\text {pos }}(\infty)=-R\left(x_{\mathrm{A}} \ln x_{\mathrm{A}}+x_{\mathrm{v}} \ln x_{\mathrm{v}}\right)$, where $x_{\mathrm{A}}=1 /\left(1+\Delta V_{\mathrm{m}} / V_{\mathrm{m}}\right)$ with $\Delta$ denoting the difference during the melting and $x_{\mathrm{v}}=1-x_{\mathrm{A}}$ being the molar fractions of the host material and vacancies, respectively. For metallic crystals, the type of chemical connection does not change during the melting transition. Thus, $S_{\mathrm{el}}(\infty) \approx 0[54]$ and $S_{\mathrm{vib}}(\infty)=S_{\mathrm{m}}(\infty)-S_{\mathrm{pos}}(\infty)$, or,

$$
S_{\mathrm{vib}}(\infty)=S_{\mathrm{m}}(\infty)+R\left(x_{\mathrm{A}} \ln x_{\mathrm{A}}+x_{\mathrm{v}} \ln x_{\mathrm{v}}\right)
$$

For semi-metals, $S_{\mathrm{el}}(\infty) \neq 0$, and $S_{\mathrm{vib}}(\infty)$ must be determined in a direct way, i.e., by the use of the Mott equation [53],

$$
S_{\text {vib }}(\infty)=3 R \ln \left(v_{\mathrm{s}} / v_{\mathrm{l}}\right)=(3 / 2) R \ln \left(\mu_{\mathrm{s}} / \mu_{1}\right)
$$

where $v$ and $\mu$ denote the characteristic vibration frequency and the electrical conductivity, respectively. If the parameters in Eq. (13) are unavailable, the following equation can also be employed as a rough approximation [54],

$$
S_{\text {vib }}(\infty)=S_{\mathrm{m}}(\infty)-R
$$

For semiconductors, the melting is accompanied by a semiconductor-to-metallic transition and the elements or compounds suffer contraction in volume rather than expansion for the most metals. Thus, $S_{\mathrm{el}}(\infty)$ strongly contributes to $S_{\mathrm{m}}(\infty)$, and $S_{\text {pos }}(\infty)<S_{\mathrm{el}}(\infty) . S_{\mathrm{pos}}(\infty)$ is thus negligible as a first order approximation [55]. Namely,

$$
S_{\text {vib }}(\infty)=S_{\mathrm{m}}(\infty)-S_{\text {el }}(\infty)
$$

Note that Eq. (15) is invalid for some metallic mixing oxides, for instance, $\mathrm{Fe}_{3} \mathrm{O}_{4}$ consisting of $\mathrm{Fe}_{2} \mathrm{O}_{3}+\mathrm{FeO}$, which undergoes semiconductor-to-metal transition due to the $\mathrm{Fe}^{2+}$ and $\mathrm{Fe}^{3+}$ ions order on the $B$ sites at $122 \mathrm{~K}$ (The so called Verwey transition) while $T_{\mathrm{m}}$ is far above the Verwey temperature [56].

\subsubsection{Verification of Model}

Fig. (1a-c) present $T_{\mathrm{m}}(D)$ and $\Theta_{\mathrm{D}}(D)$ functions of magnetic nanocrystals in terms of Eq. (9): $T_{\mathrm{m}}(D)$ and $\Theta_{\mathrm{D}}(D)$ functions decrease as $D$ is reduced or surface/volume ratio is increased due to higher energetic state of surface atoms [5]. Note that at the same $D$, different surface/volume ratios of nanocrystals with different $d$ are different. Thus, The drops of $T_{\mathrm{m}}(D)$ and $\Theta_{\mathrm{D}}(D)$ functions increases as $d$ decreases. The agreements between model predications in terms of Eq. (9) and available experimental evidence for magnetic $\mathrm{Fe}, \mathrm{Co}$, Ni nanocrystals with different dimensions $d=0,1,2$ confirm the validity of the proposed model.

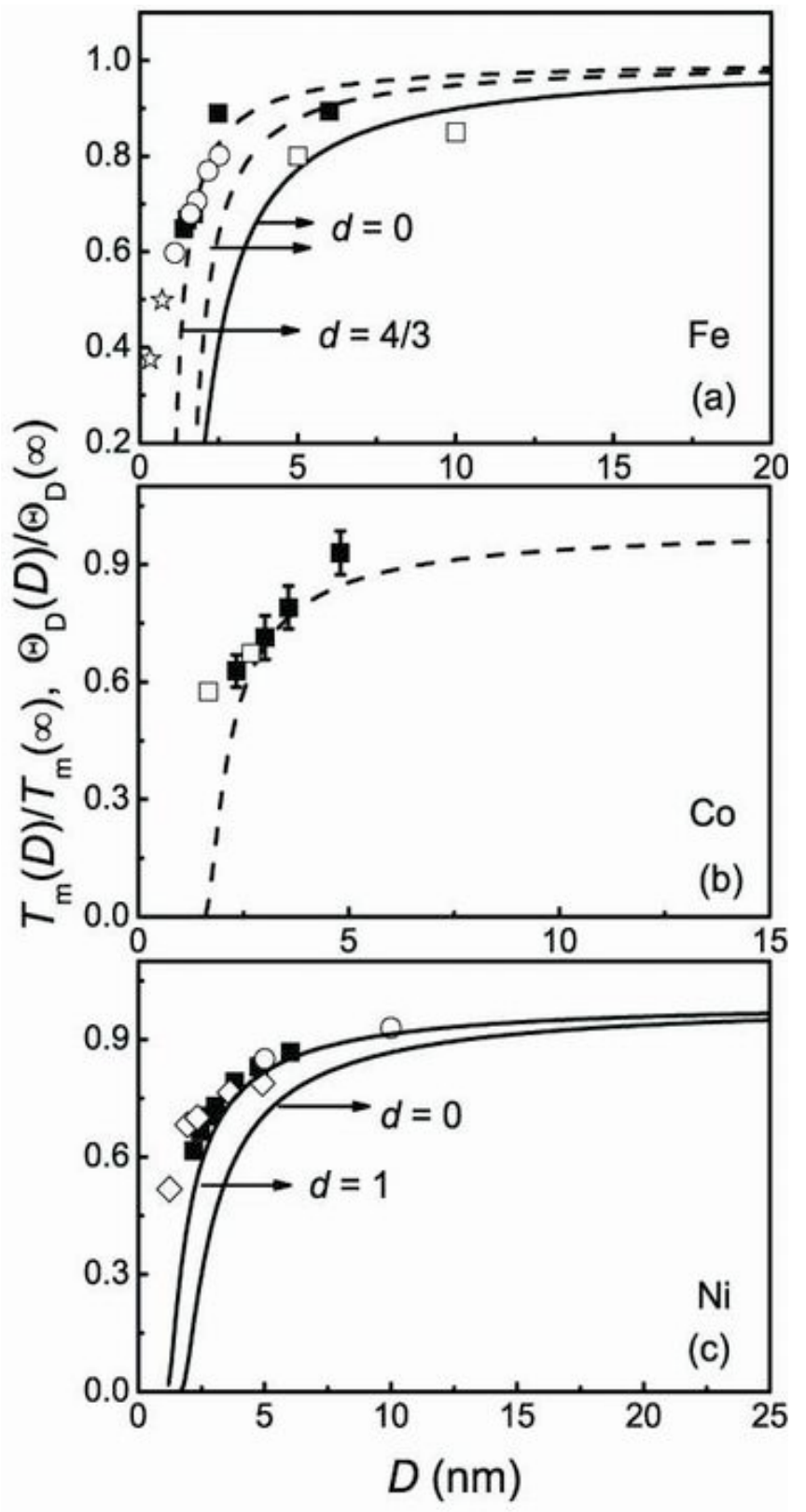

Fig. (1). Comparisons of $T_{\mathrm{m}}(D)$ (solid lines) and $\Theta_{\mathrm{D}}(D)$ (dashed lines) functions obtained from Eq. (9) and available experimental evidences for (a) Fe, (b) Co and (c) Ni nanocrystals corresponding to different $d$ values. The parameters utilized in Eq. (9) are: (a) for Fe $D_{0}=1.489$ and $0.8273 \mathrm{~nm}$ determined by Eq. (8) with $d=0$ and $4 / 3$ for nanoparticles and thin granular films, respectively, $\alpha=1.612$ in terms of Eq. (11), and the symbols $O$ [17], is [22] $\mathbf{0}$ [23] denote the experimental $\Theta_{\mathrm{D}}(D)$ data of Fe nanoparticles and thin granular films, respectively, and $\square$ [20] shows experimental measurement of $T_{\mathrm{m}}(D)$ of Fe nanoparticles; (b) for Co $D_{0}=1.504$ according to Eq. (8) with $d=0$ for nanoparticles, $\alpha=1.734$ in terms of Eq. (11), the symbols $\square$ and $\square$ [24] denote the experimental results of $\Theta_{\mathrm{D}}(D)$ of Co nanoparticles; (c) for Ni $D_{0}=1.495$ and $0.9968 \mathrm{~nm}$ according to Eq. (8) with $d=0$ and 1 for nanoparticles and nanorods, respectively, $\alpha=1.811$ in terms of Eq. (11), the symbols $\bigcirc$ [18], $\diamond$ [19], $\mathbf{\square}$ [21], denote the $T_{\mathrm{m}}(D)$ of Ni nanoparticles and nanorods, respectively. The other parameters are listed in Table $\mathbf{1}$. 
Table 1. The Parameters Employed in the Calculations of Eq. (9). $T_{\mathrm{m}}(\infty)$ and $\Theta_{\mathrm{D}}(\infty)$ are in $\mathrm{K}, S_{\mathrm{m}}(\infty)$ in $\mathrm{J} \cdot \mathrm{g}-\operatorname{atom}^{-1} \cdot \mathbf{K}^{-1}$, and $h$ is in $\mathbf{n m}$

\begin{tabular}{|c|c|c|c|c|}
\hline & $\boldsymbol{T}_{\mathbf{m}}(\infty)[\mathbf{5 7}]$ & $\boldsymbol{\Theta}_{\mathbf{D}}(\infty)[\mathbf{5 8}]$ & $\boldsymbol{S}_{\text {vib }}(\infty)[\mathbf{5 8}]$ & $\boldsymbol{h}[\mathbf{5 7}]$ \\
\hline \hline $\mathrm{Fe}$ & 1811 & 388 & 7.628 & 0.2482 \\
\hline $\mathrm{Co}$ & 1768 & 400 & 9.157 & 0.2507 \\
\hline $\mathrm{Ni}$ & 1726 & & 10.12 & 0.2492 \\
\hline
\end{tabular}

The granular films cannot be described as a continuous film [48] since they are of high densities of grain boundaries. This granular structure is similar to a random structure of a chain polymer modelled by a trajectory of a self-avoiding walk with a fractal dimension of $d^{\prime}=(2+d) / 3$ [48]. For thin films, since $d=2, d^{\prime}=4 / 3$ in terms of the above equation. This can be observed in Fig. (1a), where $\Theta_{\mathrm{D}}(D)$ functions (dashed lines) of Fe granular thin films are present.

\subsection{Mechanical Properties of Magnetic Nanocrystals}

In addition to the depression of thermal stability, nanocrystals may also exhibit increased strength/hardness, improved toughness, as well as reduced elastic modulus and ductility [59]. In the conventional polycrystalline range, the yield strength $\chi$ or hardness $H$ increases with decrease in grain size $D$, namely the Hall-Petch relationship [60]. From a practical point of view, the mechanical properties of magnetic nanocrystals often dictate whether a special need or application can be met. Therefore, a number of experimental measurements of $\chi$ or $H$ [61-67], and elastic modulus [68,69] of $\mathrm{Fe}$ and $\mathrm{Ni}$ nanocrystals have been carried out. In light of the Hall-Petch relationship [59,70,71],

$$
\chi(D)=\chi_{0}+k_{\mathrm{d}} D^{-n}
$$

where $n$ has a type value of $0.5, \chi_{0}$ is the bulk strength, and $k_{\mathrm{d}}$ is a positive material constant. Eq. (16) corresponds to experimental results when $10 \mu \mathrm{m}>D>100 \mathrm{~nm}$. As $D<100$ $\mathrm{nm}$, such as $10-30 \mathrm{~nm}$ depending on the nature of the materials, error is present [6,59,61-67]. When the yield strength $\chi$ reaches a maximun at about $D=10 \mathrm{~nm}$, then, a negative slope as the inversed Hall-Petch relationship is present $[6,59,72]$.

The above disagreement is induced by the neglect of temperature dependence of Eq. (16). In Eq. (16), the experimental temperature is usually room temperature $T_{\mathrm{a}}[63,65]$ while in Hall and Petch investigations, $100 \mathrm{~nm}$ is almost the smallest $D$ where $T_{\mathrm{m}}(D)$ is size-independent [18,19,21,22]. Both conditions result in $T_{\mathrm{a}} / T_{\mathrm{m}}(D)$ to be a constant. As $D<$ $100 \mathrm{~nm}, T_{\mathrm{m}}(D)$ evidently drops [18-21] and $T_{\mathrm{a}} / T_{\mathrm{m}}(D)$ increases where the crystals start to behave high temperature properties or grain boundary movement contributes plastic deformation [6]. As a result, $\chi_{0}$ and $k_{\mathrm{d}}$ are temperature or size dependent. As $D$ further decreases, a negative $k_{\mathrm{d}}$ has been reported due to the temperature effect $[66,73,74]$.

\subsubsection{Model for the Inversed Hall-Petch Relationship}

When $D$ is a constant, let $\chi_{0}^{\prime}$ denote the effect of $T_{\mathrm{a}}$ on $\chi$, which is given as [75]

$$
\chi_{0}{ }^{\prime}=k_{\mathrm{t}}{ }^{\prime} \exp \left[Q_{\mathrm{a}}(D) /\left(2 R T_{\mathrm{a}}\right)\right]
$$

where $k_{\mathrm{t}}^{\prime}$ is a constant, $Q_{\mathrm{a}}(D)$ is the activation energy for interface migration. Because the activation entropy induced by vibrational frequency change due to the activation process is less than $5 \%$, which is rather small, even if $D$ varies from bulk to 2-3 nm [76-78], $Q_{\mathrm{a}}(D) \propto R T_{\mathrm{m}}(D)$ where $T_{\mathrm{m}}(D)$ has been used to substitute the original $T_{\mathrm{m}}(\infty)$ [76]. As a first order approximation, it is assumed that $\chi_{0}=\chi_{0}{ }^{\prime \prime}+\chi_{0}{ }^{\prime}$, or $\chi_{0}$ is a sum of the temperature-independent term and the temperature-dependent term. Accordingly, substituting the above equation into Eq. (17) and combining the coefficient of the above equation into $k_{\mathrm{t}}{ }^{\prime}$ of Eq. (17), it reads,

$$
\chi_{0}=\chi_{0}{ }^{\prime \prime}+k_{\mathrm{t}}{ }^{\prime} \exp \left[T_{\mathrm{m}}(D) /\left(2 T_{\mathrm{a}}\right)\right]
$$

If $k_{\mathrm{d}}$ in Eq. (16) has the same temperature dependence as in Eq. (17), $k_{\mathrm{d}}=k_{\mathrm{d}}^{\prime} \exp \left[\left(T_{\mathrm{m}}(D) / 2 T_{\mathrm{a}}\right)\right]$. Because the migration resistance of dislocations should have also an Arrhenius relationship with $T$ [61], substituting Eqs. (9), (18) and the above equation into Eq. (16) with $n=1 / 2$,

$$
\begin{aligned}
& \chi(D)=\chi_{0}^{\prime \prime}+\left(k_{\mathrm{t}}{ }^{+}+k_{\mathrm{d}} D^{-1 / 2}\right) \exp \left\{\left[T_{\mathrm{m}}(\infty) /\left(2 T_{\mathrm{a}}\right)\right] \exp \right. \\
& \left.\left[-2 S_{\mathrm{m}}(\infty) /[(3 R)(D / 6 h-1)]\right]\right\}
\end{aligned}
$$

When $D>10 \mathrm{~nm}$, in terms of mathematic expression: $\exp (-x) \approx 1-x$ when $x$ is small, the double exponential term in Eq. (19), $\exp \left[-2 S_{\mathrm{m}}(\infty) /[(3 R)(D / 6 h-1)]\right] \approx 1-2 S_{\mathrm{m}}(\infty) /[(3 R)(D /$ $6 h-1)]$. Accordingly, Eq. (19) is rewritten as,

$$
\chi(D)=\chi_{0}{ }^{\prime \prime}+\left(k_{\mathrm{t}}+k_{\mathrm{d}} D^{-1 / 2}\right) \exp \left[-H_{\mathrm{m}}(\infty) /\left[3 R T_{\mathrm{a}}(D / 6 h-1)\right]\right]
$$

with $k_{\mathrm{t}}=k_{\mathrm{t}}^{\prime} \exp \left[T_{\mathrm{m}}(\infty) /\left(2 T_{\mathrm{a}}\right)\right], \quad k_{\mathrm{d}}=k_{\mathrm{d}}^{\prime} \exp \left[T_{\mathrm{m}}(\infty) /\left(2 T_{\mathrm{a}}\right)\right]$, $H_{\mathrm{m}}(\infty)=T_{\mathrm{m}}(\infty) S_{\mathrm{m}}(\infty)$. As $D \rightarrow \infty$, it leads to,

$$
\chi_{0}=\chi_{0}^{\prime \prime}+k_{\mathrm{t}}
$$

and,

$$
k_{\mathrm{d}}{ }^{\prime}=k_{\mathrm{d}} \text {. }
$$

To determine the relative size of $\chi_{0}^{\prime \prime}$ and $k_{\mathrm{t}}$, let $d \chi(D) / d\left(D^{-1 / 2}\right)=0$ in Eq. (20), where

$\frac{k_{t}}{k_{d}}=\frac{T_{a} R}{4 h H_{m}} D_{\max }^{1 / 2}-\frac{3 T_{a} R+H_{m}}{H_{m}} D_{\max }^{-1 / 2}+\frac{9 h T_{a} R}{H_{m}} D_{\max }^{-3 / 2}$

and $\chi=\chi_{\max }$ with the subscript max denoting the maximum. Since the most experimental values of $D_{\max }{ }^{-1 / 2}$ are between 0.2 and 0.4 , while $h \approx 0.3 \mathrm{~nm}, H_{\mathrm{m}}(\infty) /\left(T_{\mathrm{a}} R\right) \approx 4-7\left(T_{\mathrm{a}}=300\right.$ $\mathrm{K})$,

$$
k_{\mathrm{t}} / k_{\mathrm{d}} \approx 0-0.1
$$

With Eqs. (21)-(23), the fitting parameters among $\chi_{0}{ }^{\prime \prime}, k_{\mathrm{d}}$ and $k_{\mathrm{t}}$ in Eq. (20) are comparable with the original fitting parameters of $\chi_{0}$ and $k_{\mathrm{d}}$ in Eq. (16). This comparison can be used to verify the fitting parameters of Eq. (20). 


\subsubsection{Verification of Model}

Fig. (2a,b) show the comparisons of $\chi(D)$ and $H(D)$ functions of $\mathrm{Fe}$ and $\mathrm{Ni}$ between Eq. (20) and experimental data, respectively. When experimental $\chi_{0}$ values are known, they are used in our fitting. However, our estimated $k_{\mathrm{d}}$ values more or less differ from the corresponding literatures due to different function forms between Eq. (16) and Eq. (20) as shown in the corresponding figure captions. To clarify, the fitted parameters from literatures in terms of Eq. (16) are shown as dashed lines in the figure. Although our fitting results with the above phenomenological model are only roughly in agreement with experimental results, the tendency that there is a maximum in $\chi(D)$ and $H(D)$ functions in the range of 15-30 nm implies that the interplay between dislocation and grain-boundary processes occurs during deformation and their contributions on $\chi$ vary as size changes [7982]. Namely, as $D$ decreases to about $10 \mathrm{~nm}$, grain-boundary weakening is present [82] and $\chi(D)$ and $H(D)$ values drop gradually.

For large $D$, Eq. (20) can be approximated by $\chi(D) \approx$ $\chi_{0}{ }^{\prime \prime}+k_{\mathrm{t}}+k_{\mathrm{d}} D^{-1 / 2}-\left[2 h H_{\mathrm{m}} /(R T a)\right]\left[k_{\mathrm{Ta}}{ }^{-1}+k_{\mathrm{d}} D^{-3 / 2}\right]$ where $k_{\mathrm{d}}$ appears both in $D^{-1}$ term and $D^{-3 / 2}$ term. The latter should denote the temperature effect. Since $k_{\mathrm{d}}>100 k_{\mathrm{t}}, k_{\mathrm{t}}$ as a coefficient of $D^{-1}$ has little effect on $\chi(D)$. Note also that $\chi_{0}{ }^{\prime \prime}>10 k_{\mathrm{t}}$ (see the captions of the figures), a weak effect of $k_{\mathrm{t}}$ on $\chi_{0}$ is also evident. Thus, the change of $\chi(D)-D^{-1 / 2}$ curve in Eq. (16) is essentially induced by temperature effect on $k_{\mathrm{d}}$. This analysis leads to a further simplification of the above equation,

$$
\chi(D) \approx \chi_{0}+k_{\mathrm{d}} D^{-1 / 2}(1-c / D)
$$

where $c=2 h H_{\mathrm{m}} /(R T a), \chi_{0}=\chi_{0}{ }^{\prime \prime}+k_{\mathrm{t}}$, and $k_{\mathrm{d}}{ }^{\prime}=k_{\mathrm{d}}$. Comparing Eqs. (16) and (24), Eq. (16) has neglected the term of $c D^{-3 / 2}$. Since $c<10 \mathrm{~nm}$, Eq. (16) has little error when $D>100 \mathrm{~nm}$. Eq. (24) implies that there is no maximum in $\chi(D)$ curve at $D>100 \mathrm{~nm}$.

The Eq. (20) indicates that while $T_{\mathrm{a}}$ is a constant, a material with a higher $H_{\mathrm{m}}$ value has a larger $\chi_{\max }$, yet a smaller $D_{\max }{ }^{-1 / 2}$, and a stronger grain boundary strengthening as $D$ decreases, which in turn leads to an early grain boundary softening at larger $D$ value.

\section{FERROMAGNETIC PROPERTIES}

Origin of ferromagnetism is the interplay among the electronic spin degree of freedom, the repulsive Coulomb interactions among electrons, and the fermionic quantum statistics of electrons [83]. Because magnetic order is associated with the strong repulsive Coulomb interactions among electrons, it can persist to a very high temperature. Below the temperature, very often the quantum ground state of a manyelectron system has nonzero local spin density, which is aligned either in the same direction in space at every point in the system (like simple ferromagnets), or is noncollinear, such as ferrimagnetic, or antiferromagnetic materials in configurations, in which the spin direction varies spatially [83].

The density-function theory can be utilized due to increased computer power to illustrate the strong-coupling
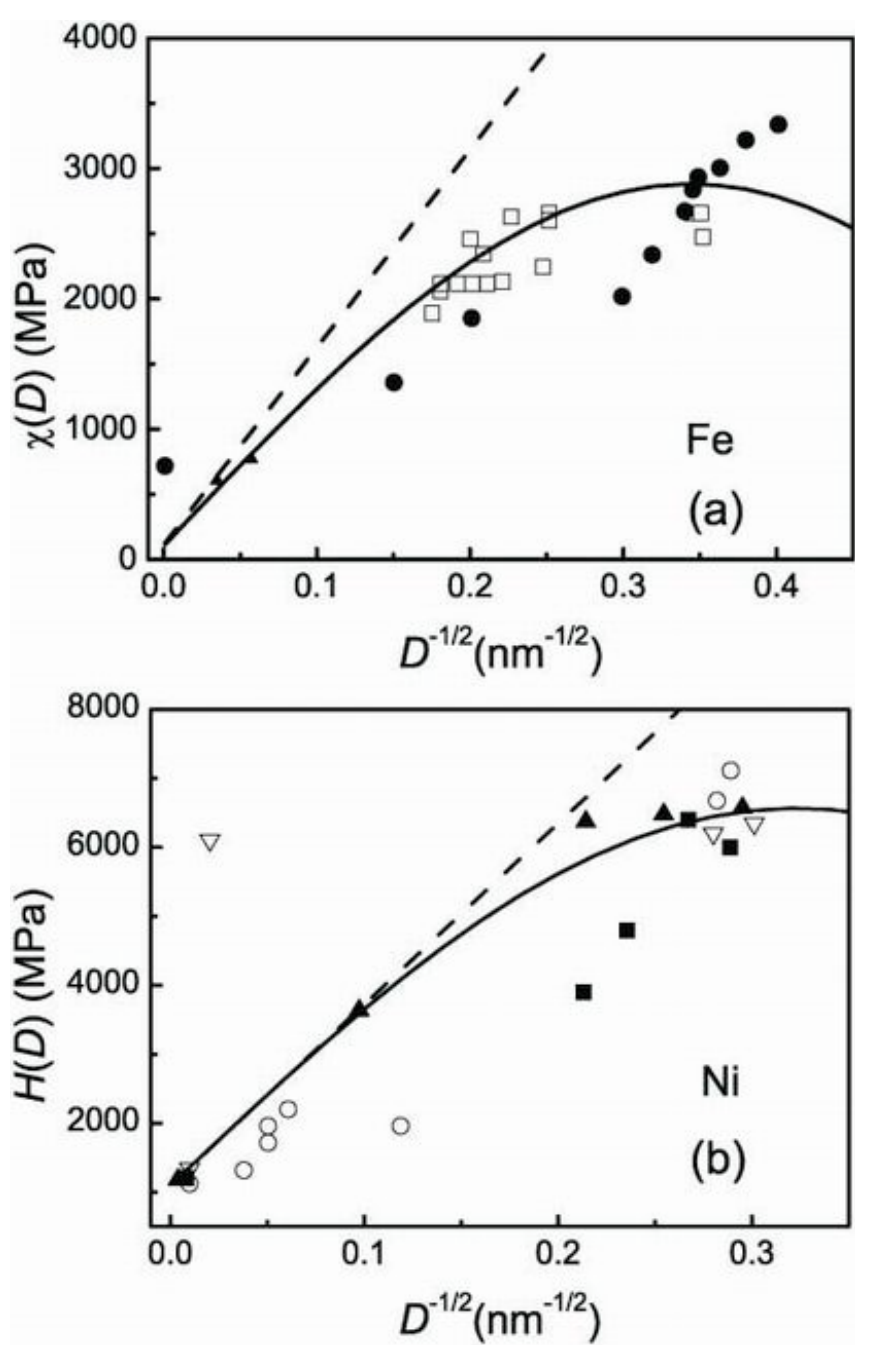

Fig. (2). Comparison of Hall-Petch relationship of (a) $\mathrm{Fe}$ and (b) $\mathrm{Ni}$ nanocrystals between Eq. (20) (solid line) and experimental results shown as: (a) for $\mathrm{Fe} \bullet$ [63] (the original $H$ data is transformed to $\chi$ by $\chi=H / 3) \square$ [64], $\boldsymbol{\Delta}$ [64]; (b) for $\mathrm{Ni} \boldsymbol{\Delta}$ [65] $\bigcirc, \nabla$ [66] and $\boldsymbol{\square}$ [67]. The necessary parameters are listed as: (a) for Fe nanocrystals, $\chi_{0}=103 \mathrm{MPa}$ and $k_{\mathrm{d}}=15178 \mathrm{MPa} \mathrm{nm}^{1 / 2}$ [64], and the obtained fitting parameters are $\chi_{0}{ }^{\prime \prime}=75 \mathrm{MPa}, k_{\mathrm{t}}=28.5 \mathrm{MPa}$ and $k_{\mathrm{d}}=12437$ $\mathrm{MPa} \mathrm{nm}{ }^{1 / 2}$; (b) for Ni nanocrystals, $k_{\mathrm{d}}=26281 \mathrm{MPa} \mathrm{nm}^{1 / 2}[65]$ and $\chi_{0}{ }^{\prime \prime}=1021 \mathrm{MPa}, k_{\mathrm{t}}=71 \mathrm{MPa}$ and $k_{\mathrm{d}}=26632 \mathrm{MPa} \mathrm{nm}^{1 / 2}$. Note that $k_{\mathrm{d}}$ in experiments [64] differs about $18 \%$ and $1 \%$ from our fitted $k_{\mathrm{d}}$ values for $\mathrm{Fe}$ and $\mathrm{Ni}$, respectively. The dash lines show Eq. 16 with experimental values of $\chi_{0}$ and $k_{\mathrm{d}}[64,65]$. Other necessary parameters in Eq. (20) see Fig. (1). Note that the flaws (such as voids, microcracks, incomplete boundaries, etc.) in nanocrystals affect $\chi$ values. Newer experimentals results thus are taken into account since they have better avoided them [59,61,63,66,73].

phenomenon of ferromagnetism, which includes the spindensity-functional generalization where energy functions depend on charge and spin densities [83]. Although densityfunction theory is exact in principle, its application requires approximation of exchange-correlation energy, which can partially be phenomenological and are normally based in part on microscopic calculations of correlation effects in the electron-gas model system $[83,84]$.

The Weiss theory of the molecular field published in 1907 is the key development in phenomenological descrip- 
tion of ferromagnetism [85]. Although Weiss himself did not make any specific prediction about the origin of the molecular field in terms of atomic forces, but he did explain the principal aspects of ferromagnetism by means of two assumptions of existences of the molecular field and domain structure. In 1926, on the basis of the prerequisite that the Sspin is $T$-independent and magnetic field independent, Heisenberg interpreted the molecular field in terms of exchange forces, namely the Heisenberg model [86], which extends description of the magnetic properties also for the $3 \mathrm{~d}$ transition metal magnets ( $\mathrm{Cr}$ to $\mathrm{Ni}$ ) and their compounds.

In terms of the Heisenberg model, the total energy of magnetic body consists of the exchange interaction energy $E_{\text {exc }}$, the anisotropy energy $E_{\text {ani }}$, the magnetostatic energy $E_{\mathrm{s}}$ and the Zeeman energy, $E_{z}$ [87]. The magnetic configuration is determined by minimizing the total magnetic energy. $E_{\text {exc }}$ originates from the Coulomb interaction under consideration of the Pauli exclusion principle and the antisymmetry of the total electronic wave function [87], which is usually written as [88],

$$
E_{\text {exc }}=-2 \sum J S_{\mathrm{i}} \cdot S_{\mathrm{j}}
$$

where $J$ is the exchange constant or exchange coefficient between atoms $i$ and $j$, and $S_{\mathrm{i}}$ is the S-spin of atom $i$. In the most cases, it is sufficient to consider only the exchange interaction between spins on nearest-neighbor atoms. $J$ is strongly related to the overlap of the two atoms and hence to $h$, or $J=J(h)$. The $J$ of $3 \mathrm{~d}$ metals is big where there is a large extent of 3d-electron charges [89,90], which is confirmed by the quantum-mechanical calculations based on the HeitlerLondon approach [91]. An example of the Bethe-Slater curve is shown in Fig. (3) where $r$ denotes the radius of the incompletely filled $d$ shell. As indicated in the figure, a large value of $h / r$ corresponds to a positive $J$ like ferromagnetic $3 \mathrm{~d}$ elements $\mathrm{Ni}, \mathrm{Co}, \mathrm{Fe}$ (paraller moment arrangements), whereas for small values it was negative like antiferromagnetic elements $\mathrm{Mn}$ and $\mathrm{Cr}$ (antiparallel moment arrangements).

Due to the isotropic nature of exchange interaction, exchange interaction does not force the spins to turn to a certain direction. $E_{\text {exc }}$ are scaled by the exchange length $L_{\text {exc }}$ [86],

$$
L_{\mathrm{exc}}=\left[2 A /\left(\mu_{0} M_{\mathrm{s}}^{2}\right)\right]^{1 / 2}
$$

with $A=2 J S_{\mathrm{i}} S_{\mathrm{j}} / a$ being the exchange stiffness, where $a$ is the lattice constant, $\mu_{0}$ is the permeability of the vacuum, and $M_{\mathrm{s}}$ the saturation magnetization.

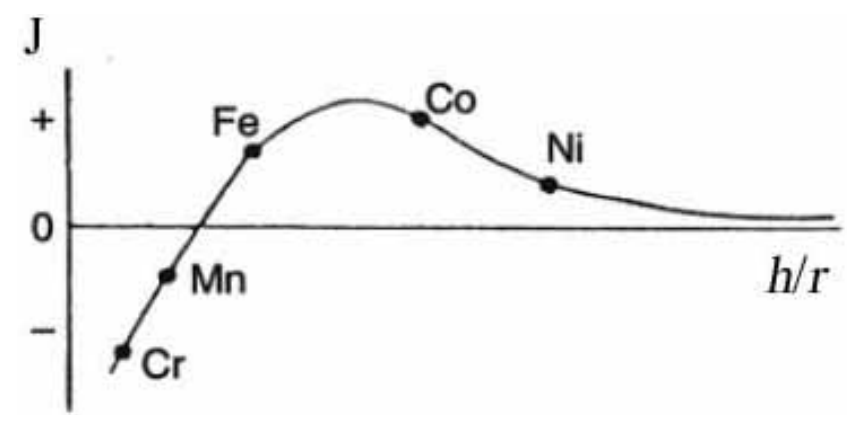

Fig. (3). A schematic plot of the exchange constant $v$ s interatomic distance [88].
$E_{\text {ani }}$ denotes the energy that directs the magnetization along certain crystallographic axes (the easy axes of magnetization) [86], which is a small fraction of the total energy per atom in solids ( $\approx 10 \mathrm{eV} /$ atom) but important [92], and is scaled by anisotropy length or the domain wall width $L_{\mathrm{w}}$,

$$
L_{\mathrm{w}}=(A / K)^{1 / 2}
$$

where $K$ is the anisotropy constant. $E_{\text {ani }}$ increases when the magnetic moment deviates from the easy direction.

In comparison with $E_{\mathrm{exc}}, E_{\mathrm{s}}$ is induced by dipole-dipole interactions and is smaller but long ranged. If the magnetic domain configuration entails a null net magnetic moment, $E_{\mathrm{s}}$ is minimized, for the example of magnetic vortex state, in which the magnetic stray field essentially vanishes with $E_{\mathrm{s}}=$ 0 . In addition, $E_{\mathrm{z}}$ is negligible for the remanence state.

When $D$ is comparable with characteristic lengthes such as $L_{\mathrm{w}}$ and $L_{\mathrm{exc}}$, which are about several and several ten nanometers, respectively, the magnetic properties of nanocrystals, such as the magnetization, the order temperature, the magnetic moment and the coercivity, dramatically deviate from the bulk counterparts, which are reviewed in this section.

\subsection{Size Effects on Phase Stability of FM Nanocrystals}

\subsubsection{Experiment Results}

The phase stability of FM nanocrystals, denoted by the Curie temperature $T_{\mathrm{c}}$, is an important physical quantum to characterize FM. $T_{\mathrm{c}}(D)$ functions of $\mathrm{Fe}$, Co, and Ni transition nanocrystals, rare-earth nanometals like $\mathrm{Gd}$ and $\mathrm{Tb}$, and their alloys and compounds have been investigated experimentally. Commonly, the monolayer or multilayer magnetic films are epitaxially grown on or between nonmagnetic materials like noble metals, nonmagnetic transition metals, semiconductors, as well as insulator substrates, such as $\mathrm{Fe} / \mathrm{SiO}$ [93], $\mathrm{Fe} / \mathrm{Cu}(001)$ [94], $\mathrm{Fe} / \mathrm{Ag}(001)$ [94], $\mathrm{Fe} / \mathrm{Au}(100)$ [95], $\mathrm{Fe} / \mathrm{Pd}(100)$ [96], $\mathrm{Fe} / \mathrm{Ag}(111)$ [97], $\mathrm{Fe} / \mathrm{Ag}(100)$ [98], $\mathrm{Co} / \mathrm{Cu}(100) \quad$ [99], $\quad \mathrm{Co} / \mathrm{Cu}(111) \quad[100,101], \quad \mathrm{Co} / \mathrm{Cu}(001)$ [101,102], $\mathrm{Ni} / \mathrm{Cu}(100)$ [101], $\mathrm{Ni} / \mathrm{Cu}(001)$ [102], $\mathrm{Ni} / \mathrm{Cu}(111)$ [103], Ni/W(110) [104], $\quad \mathrm{Co}_{1} \mathrm{Ni}_{1} / \mathrm{Cu}(100) \quad$ [101], $\mathrm{Co}_{1} \mathrm{Ni}_{3} / \mathrm{Cu}(100)$ [101], $\mathrm{Co}_{1} \mathrm{Ni}_{9} / \mathrm{Cu}(100)$ [101], Gd/W(110) [105], Gd/W [106], Nb/Gd [107], and $\mathrm{Gd} / \mathrm{Y}(0001)$ [108] systems. Other low-dimensional nanocrystals, i.e. Ni nanorods $[109,110]$ and nanoparticles [110,111], $\mathrm{Gd}[112], \mathrm{Fe}_{3} \mathrm{O}_{4}$ [113], and $\mathrm{MnFe}_{2} \mathrm{O}_{4}$ [114] nanoparticles have been studied. Their structures and properties are determined by various techniques like magnetometry, spin-polarized photoemission, the magneto-optical Kerr effect, and acsusceptometry experiments, as well as more improved methods such as the ferromagnetic resonance, the conversionelectron Mössbauer spectroscopy, X-ray magnetic circular dichroism, the resonance magnetic X-ray spectroscopy, the magnetic force microscopy, etc. Generally, for free nanocrystals and thin films deposited on nonmagnetic substrates, $T_{\mathrm{c}}(D)$ drops as $D$ and $d$ are reduced. Whereas for FM nanocrystals deposited epitaxially on the magnetic substrates, enhanced $T_{\mathrm{c}}(D)$ function has been observed, for the example of $\mathrm{Ni} /$ Co heterogeneous system [115], in which $T_{\mathrm{c}}(D)$ of $\mathrm{Ni}$ increases $60 \mathrm{~K}$ as $D$ varies from 10 to $5 \mathrm{~nm}$, where $D$ of Co 
layer stays at $15 \mathrm{~nm}$. Hernando et al. introduced the concept of exchange interface thickness (EIT) to present a possible qualitative explanation [115]. EIT can be defined as that along which $J$ evolves from $J_{\mathrm{A}}$ to $J_{\mathrm{B}}$, where subscripts A and $\mathrm{B}$ denote bulk $\mathrm{A}$ and $\mathrm{B}$ phase, respectively. The characteristic of EIT is great interest to understand the magnetic behavior of multiphase magnetic system [116,117] as well as to improve understanding of exchange between ferromagnetic layers through paramagnetic spacers $[118,119]$. EIT is expected to be restricted to $h \sim 2 h$, and its influence on the macroscopic magnetic properties should be noticeable in those samples with outstanding interface area per unit volume, such as the coercivity and $T_{\mathrm{c}}$ [115].

\subsubsection{Theoretical Models}

The pioneering theoretical work for underlying mechanisms of $T_{\mathrm{c}}(D)$ function was developed by Fisher and his coworkers [120], in which the spin-spin correlation length $\xi(T)$ $=\xi_{0}\left(1-T / T_{\mathrm{c}}\right)^{-1 / \lambda}$ is defined as the distance from a point beyond which there is no further correlation of a physical property associated with that point, $\xi_{0}$ is a microscopic length, and $\lambda$ is a universal critical exponent [120]. This scaling relationship predicts a drop of $T_{\mathrm{c}}(D)$ when $\xi>D$. As far as thin films are concerned, the above mechanism has given rise to a step function for $T_{\mathrm{c}}(D)$ with two adjustable parameters $\xi$ and $\lambda$ $[120,121]$. When $d=2$ then for the case $D>\xi$

$$
T_{\mathrm{c}}(D) / T_{\mathrm{c}}(\infty)=1-[(\xi+h) /(2 D)]^{\lambda}
$$

and for the case $D<\xi$,

$$
T_{\mathrm{c}}(D) / T_{\mathrm{c}}(\infty)=(D-h) /(2 \xi)
$$

While $d=0, T_{\mathrm{c}}(D)$ function has been proposed as [122],

$$
T_{\mathrm{c}}(D) / T_{\mathrm{c}}(\infty)=1-(3 \Delta L) /(2 D)
$$

where $\Delta L$ is the thickness of surface layer of nanoparticles, which characterizes the effect of the surface layer on $T_{\mathrm{c}}(D)$ function. However, this model with a constant $\Delta L$ cannot satisfactorily describe the $T_{\mathrm{c}}(D)$ function of $\mathrm{Fe}_{3} \mathrm{O}_{4}$ nanoparticles in the full size range [122].

Sun et al. have established a unified model to consider $T_{\mathrm{c}}(D)$ by incorporating BOLS correlation mechanism into the Ising premise, which has the same form of Eq. (5) [113] as $T_{c}(D) / T_{c}(\infty)=1+\sum_{i \leq 3} n_{i}\left(z_{s v} q_{s}^{-w}-1\right)$. When $w \approx 1, T_{\mathrm{c}}(D)$ functions of free nanocrystals with different $d$ values are predicted with good correspondence of experimental results [113]. Since there is an adjustable parameter in the equation, further efforts are made to develop a model without free parameter. Based on the size-dependent cohesive energy function, a $T_{\mathrm{c}}(D)$ model for the case of $d=2$ has been given as [123],

$$
\begin{aligned}
& T_{\mathrm{c}}(D) / T_{\mathrm{c}}(\infty)=\{1-1 /[2 D /(c h)-1]\} \exp \\
& \left\{\left[-2 S_{\mathrm{b}} /(3 R)\right] /[2 D /(c h)-1]\right\}
\end{aligned}
$$

where $S_{\mathrm{b}}$ is the bulk evaporation entropy of crystals. $c_{1}$ is added as an additional condition for different surface states. For the nanocrystals with free surfaces, $c_{1}=1$. When the interface interaction between nanocrystals and the corresponding substrate is weak, such as thin films deposited on inert substrates, the film/substrate interaction is weak van der Waals forces while the inner interactions within the thin films are strong chemical bonds, $c_{1}=1 / 2$. If this strength on the interface is comparable with that within films, $c_{1}$ varies to some extent [123]. For more complicated interfaces, $c_{1}$ may be considered casewise between $1 / 2$ and 1 . Eq. (31) has presented a qualitative explanation for drop of $T_{\mathrm{c}}(D)$ function of nanocrystals with decreasing of $D$.

In addition, another theoretical model, a finite-size scaling relationship [100,101], has also been proposed for reproducing $T_{\mathrm{c}}(D)$ functions of $\mathrm{Co} / \mathrm{Ni}$ alloys,

$$
\left[T_{\mathrm{c}}(\infty)-T_{\mathrm{c}}(D)\right] / T_{\mathrm{c}}(D)=\left[\left(D-D^{\prime}\right) / \xi_{0}\right]^{-\lambda}
$$

with $D^{\prime}$ denoting a finite thickness of films at $T_{\mathrm{c}}(D)=0$. Although Eq. (32) can also fit experimental data of $\mathrm{Co}, \mathrm{Co}_{1} \mathrm{Ni}_{1}$, $\mathrm{Co}_{1} \mathrm{Ni}_{3}$ and $\mathrm{CoNi}_{9}[100,101]$, it strictly hold only in the large size limit of $D$ with the help of three fitting parameters $D^{\prime}, \xi_{0}$ and $\lambda$, which are case dependent without clear physical meanings $[100,101]$.

\subsubsection{Lattice Vibration Instability Model for $T_{c}(D)$ Func- tion}

It is well known that near $T_{\mathrm{c}}$, there exist two opposite forces: the ordering force due to the exchange interaction of magnetic moments, and the disordering force of lattice thermal vibrations. Based on the mean-field approximation [121,124],

$$
k_{\mathrm{B}} T_{\mathrm{c}}(\infty)=E_{\text {exc }}(\infty)
$$

The average thermal vibrational energy is related to $T$ by an equipartition relation of $m_{\mathrm{a}}\left(2 \pi v_{\mathrm{E}}\right)^{2} \sigma^{2}(T)=k_{\mathrm{B}} T$ [51], where $m_{\mathrm{a}}$ is the atomic mass, $v_{\mathrm{E}}$ is the Einstein frequency, and $k_{\mathrm{B}}$ is the Boltzmann constant. In terms of this relationship and Eq. (33), at $T_{\mathrm{c}}(\infty)$, the thermal vibration of atoms will destroy the magnetic ordering induced by the exchange interaction of nearest-neighbor atoms, $\sigma^{2}\left[T_{\mathrm{c}}(\infty)\right]=k_{\mathrm{B}} T_{\mathrm{c}}(\infty) /\left[m_{\mathrm{a}}\left(2 \pi v_{\mathrm{E}}\right)^{2}\right]=$ $E_{\text {exc }}(\infty) /\left[m_{\mathrm{a}}\left(2 \pi v_{\mathrm{E}}\right)^{2}\right]$. Similarly, based on Lindemann's basic assumption that melting occurs when $\sigma$ reaches a fraction of $h(f)$ at $T_{\mathrm{m}}(\infty), \sigma^{2}\left[T_{\mathrm{m}}(\infty)\right]=k_{\mathrm{B}} T_{\mathrm{m}}(\infty) /\left[m_{\mathrm{a}}\left(2 \pi v_{\mathrm{E}}\right)^{2}\right]=(f h)^{2}$ and $\Theta_{\mathrm{D}}(\infty)=f\left[T_{\mathrm{m}}(\infty) /\left(m_{\mathrm{a}} h^{2}\right)\right]^{1 / 2}[124]$. In terms of above three relationships and an assumption that $E_{\text {exc }}(\infty) /\left(2 \pi v_{\mathrm{E}} f^{2}\right)^{2}$ is equal to a constant, one can obtain [124], $T_{\mathrm{c}}(\infty) \propto \Theta_{\mathrm{D}}{ }^{2}(\infty)$. If FM nanocrystals remain the corresponding bulk structure, the equation can be extended to nanometer size, $T_{\mathrm{c}}(D) \propto$ $\Theta_{\mathrm{D}}^{2}(D)$. Thus, $T_{\mathrm{c}}(D) / T_{\mathrm{c}}(\infty)=\Theta_{\mathrm{D}}{ }^{2}(D) / \Theta_{\mathrm{D}}{ }^{2}(\infty)$. Substituting Eq. (9) into this equation leads to [124],

$$
T_{\mathrm{c}}(D) / T_{\mathrm{c}}(\infty)=E_{\text {exc }}(D) / E_{\text {exc }}(\infty)=\exp \left[-(\alpha-1) /\left(D / D_{0}-1\right)\right]
$$

For metallic or compound crystals with free surfaces, $\alpha_{\mathrm{s}}$ is determined by Eq. (11). For FM or AFM films epitaxially grown on substrates, the corresponding $\alpha_{\mathrm{i}}$ must be considered since the atomic vibration at interface differs from that at free surfaces. Based on the Ising model, let the subscript ' $i$ ' denotes the interface, 'sub' the substrate and 's' the surface, the simplest case is that only the surface/interface coupling constant $\left(J_{\mathrm{s}} / J_{\mathrm{i}}\right)$ differs from the rest [125]. For the sake of simplicity, the effect induced by EIT is neglected while $J_{\mathrm{i}}=$ $J_{\mathrm{s}}+J_{\text {sub }}$ is assumed as a first approximation. Thus, $\alpha_{\mathrm{i}}=$ 
$\sigma_{\mathrm{i}}^{2}(D) / \sigma_{\mathrm{v}}^{2}(D)=\alpha_{\mathrm{s}} \sigma_{\mathrm{i}}^{2}(D) / \sigma_{\mathrm{s}}^{2}(D)$ in light of the definition of $\alpha$ where $\alpha_{\mathrm{s}}=\sigma_{\mathrm{s}}{ }^{2}(D) / \sigma_{\mathrm{v}}{ }^{2}(D)$. Since $J \propto \varepsilon$ [125-127] while $\varepsilon \propto$ $1 / \sigma^{2}[127], \sigma^{2}(D) \propto 1 / J$. Thus, $\sigma_{\mathrm{s}}^{2}(D) \propto 1 / J_{\mathrm{s}}$ and $\sigma_{\mathrm{i}}^{2}(D) \propto$ $1 / J_{\mathrm{i}}$. As a consequence, $\sigma_{\mathrm{i}}^{2}(D) / \sigma_{\mathrm{s}}^{2}(D)=J_{\mathrm{s}} / J_{\mathrm{i}}$, or,

$\alpha_{\mathrm{i}}=\alpha_{\mathrm{s}} J_{\mathrm{s}} / J_{\mathrm{i}}$

If the effects induced by surface and interface on $T_{\mathrm{c}}(D)$ are additive, according to Eq. (34), it reads,

$$
\begin{aligned}
& T_{\mathrm{c}}(D) / T_{\mathrm{c}}(\infty)=\left\{\exp \left[-\left(\alpha_{\mathrm{s}}-1\right) /\left(D / D_{0}-1\right)\right]+\exp \left[-\left(\alpha_{\mathrm{i}}-1\right) /\right.\right. \\
& \left.\left.\left(D / D_{0}-1\right)\right]\right\} / 2
\end{aligned}
$$

Note that Eq. (36) is only used for the case of thin films while the side surfaces are neglected due to their small percentage of the total surface. For nanoparticles and nanorods, the contribution of substrates on $T_{\mathrm{c}}(D)$ is neglected due to the small fraction of interface/surface. In this case, Eq. (34) is directly used. In the following, although $T_{\mathrm{c}}(D)$ function is denoted as Eq. (36), when the considered systems are nanoparticles and nanorods, $\alpha_{\mathrm{s}}=\alpha_{\mathrm{i}}$, and thus Eq. (34) $=\mathrm{Eq}$. (36) [124].

\subsubsection{Verification of $T_{c}(D)$ Function}

Fig. (4a-d) present comparisons of $T_{\mathrm{c}}(D)$ functions predicted from Eq. (36) and available experimental evidence for $\mathrm{Ni}, \mathrm{Fe}_{3} \mathrm{O}_{4}$ and $\mathrm{MnFe}_{2} \mathrm{O}_{4}$ nanoparticles, Ni nanorods, and $\mathrm{Fe}$, $\mathrm{Co}, \mathrm{Co}_{1} \mathrm{Ni}_{1}, \mathrm{Co}_{1} \mathrm{Ni}_{3}, \mathrm{Co}_{1} \mathrm{Ni}_{9}$, Ni thin films deposited on an nonmagnetic metal substrates. For an epitaxial FM film on a magnetic inert substrate with negligible lattice mismatch, exchange interaction between them is assumed to be absent because the surface and the film/substrate interface are magnetically similar $[123,124,128]$. Thus, $\alpha_{i}=\alpha_{s}$ in terms of Eq. (35) with $J_{\mathrm{i}}=J_{\mathrm{s}}$ and $J_{\text {sub }}=0$. On the other side, for this kind of epitaxial films, the interaction strength at the film/substrate interface is comparable with the inner one $[123,124]$, which results in disappearance of the interface in the above meaning. Thus, the critical size of the epitaxial films is $D_{0} / 2[123,124,127]$.

As shown in this figure, Eq. (36) is quantitatively consistent with the experimental results. The good agreement between Eq. (36) and the experimental data implies that the depression of $T_{\mathrm{c}}(D)$ of $\mathrm{Fe}$, Co and $\mathrm{Ni}$, CoNi alloy epitaxial films on nonmagnetic metallic substrates should mainly be

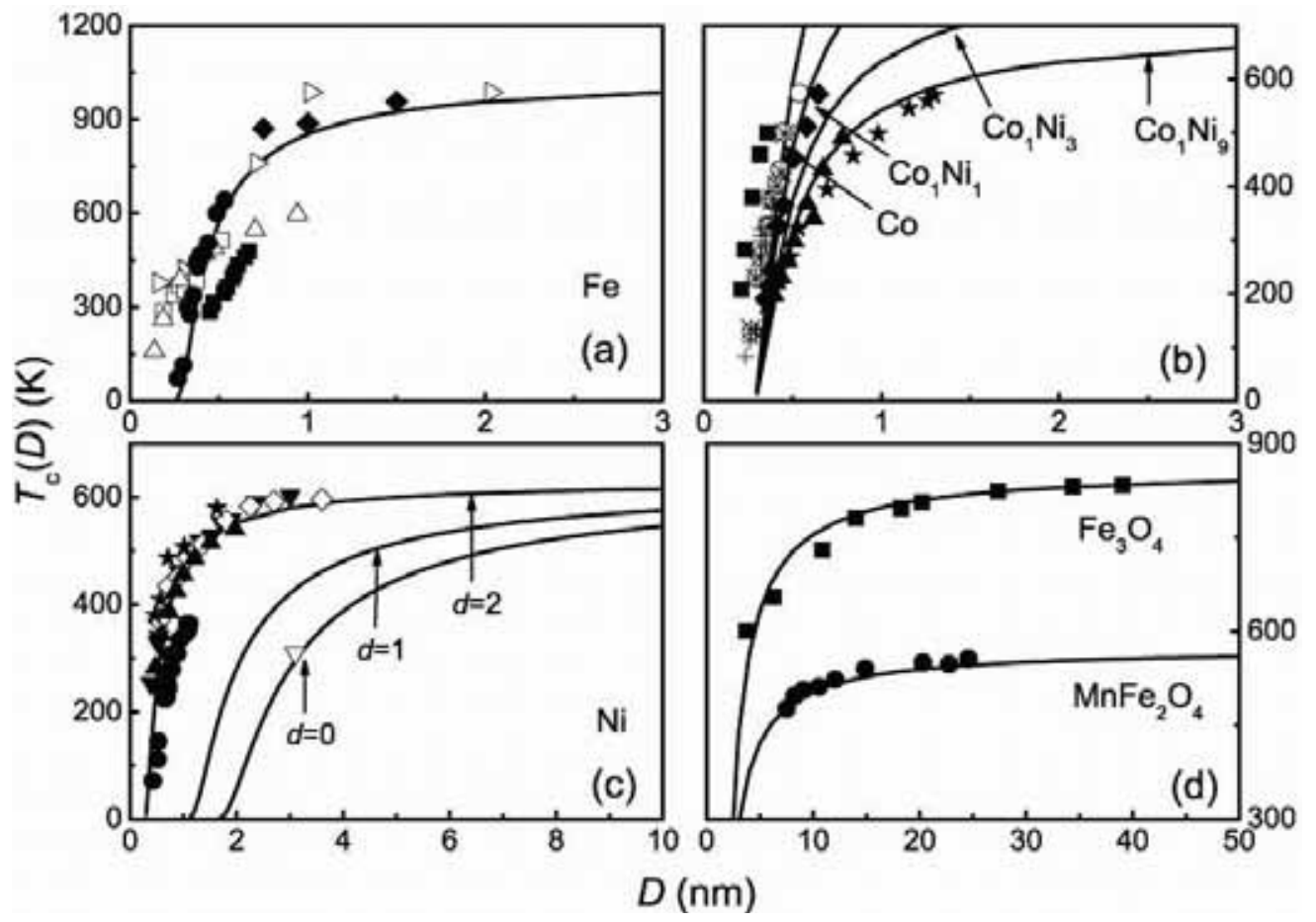

Fig. (4). Comparisons of $T_{\mathrm{c}}(D)$ functions between Eq. (36) and available experimental measurements for $\mathrm{Fe}_{2} \mathrm{Co}_{,} \mathrm{Co}_{1} \mathrm{Ni}_{1}, \mathrm{Co}_{1} \mathrm{Ni}_{3}, \mathrm{Co}_{1} \mathrm{Ni}_{9}$, $\mathrm{Ni}, \mathrm{Fe}_{3} \mathrm{O}_{4}$ and $\mathrm{MnFe}_{2} \mathrm{O}_{4}$ nanocrystals. The necessary parameters are: (a) For Fe films, $D_{0}=(2 h) / 2=0.2483 \mathrm{~nm}$ in terms of Eq. (8) with $d=2$,

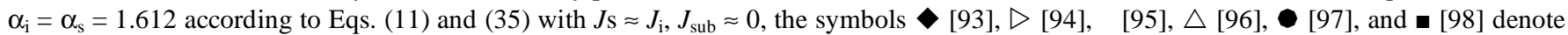
the experimental results of $\mathrm{Fe} / \mathrm{SiO}, \mathrm{Fe} / \mathrm{Ag}(001), \mathrm{Fe} / \mathrm{Au}(100), \mathrm{Fe} / \mathrm{Pd}(100), \mathrm{Fe} / \mathrm{Ag}(111)$ and $\mathrm{Fe} / \mathrm{Ag}(100)$ epitaxial films, respectively; (b) for $\mathrm{Co}, \mathrm{Co}_{1} \mathrm{Ni}_{1}, \mathrm{Co}_{1} \mathrm{Ni}_{3}$ and $\mathrm{Co}_{1} \mathrm{Ni}_{9}$ alloy films, $D_{0}=(2 h) / 2=h=0.2497,0.2495,0.2493$ and $0.2493 \mathrm{~nm}$ in terms of Eq. (8) with $d=2$, and $\alpha_{\mathrm{i}}=$ $\alpha_{\mathrm{s}}=1.734,1.773,1.792$ and 1.803 according to Eqs. (11) and (35) with $J_{\mathrm{s}} \approx J_{\mathrm{i}}, J_{\text {sub }} \approx 0$, the symbols $\bigcirc$ [99], $*$ [100,101], $\mathbf{\square}$ [101], $+[102]$ denote the experimental evidences of $\mathrm{Co} / \mathrm{Cu}(100), \mathrm{Co} / \mathrm{Cu}(111), \mathrm{Co} / \mathrm{Cu}(001)$ epitaxial films and $\bullet$ [101], $\boldsymbol{\Delta}$ [101] and $\star$ [101] denote the $\mathrm{Co}_{1} \mathrm{Ni}_{1} / \mathrm{Cu}(100), \mathrm{Co}_{1} \mathrm{Ni}_{3} / \mathrm{Cu}(100), \mathrm{Co}_{1} \mathrm{Ni}_{9} / \mathrm{Cu}(100)$ epitaxial thin films; (c) for Ni nanocrystals, $D_{0}=1.4952,0.9968,0.2492 \mathrm{~nm}$ in term of Eq. (8) with $d=0,1,2$, and $\alpha_{\mathrm{s}}=1.811$ in light of Eq. (11) for nanoparticles and nanorods, and $\alpha_{\mathrm{i}}=\alpha_{\mathrm{s}}=1.811$ in terms of Eq. (35) with $J_{\mathrm{s}} \approx J_{\mathrm{i}}$ and $J_{\text {sub }} \approx 0$ for epitaxial films on inert substrates. The symbols $\diamond$ [109], $*$ [110] denote Ni nanoparticles, $\bigcirc$ [110], [ [111], $\nabla$ [112] the nanorods, and $\boldsymbol{\Delta}$ [101], [102], $\star$ [102] $\boldsymbol{\nabla}$ [103], and $\diamond$ [104] denote the experimental evidences of $\mathrm{Ni} / \mathrm{Cu}(100), \mathrm{Ni} / \mathrm{Cu}(001), \mathrm{Ni} / \mathrm{Cu}(111)$, $\mathrm{Ni} / \mathrm{W}(110)$ epitaxial films; (d) for $\mathrm{Fe}_{3} \mathrm{O}_{4}$ and $\mathrm{MnFe}_{2} \mathrm{O}_{4}, D_{0}=1.338,1.332 \mathrm{~nm}$ in terms of Eq. (8) with $d=0$, and $\alpha_{\mathrm{s}}=1.8458,1.8458$ in terms of Eq.(11), the symbols - [113] and [114] denote $\mathrm{Fe}_{3} \mathrm{O}_{4}$ and $\mathrm{MnFe}_{2} \mathrm{O}_{4}$ nanoparticles, respectively. The other necessary parameters used in the calculations are listed in Table 2. 
attributed to the free surface effect of nanocrystals because of the reduction in the spin interaction number at the surface in comparison with that in the interior [113,121-124]. Although Eq. (5) and Eqs. (28)-(30) can also fit the experimental results of FM nanocrystals [113,121-123], some adjustable parameters, such as $w$ in Eq. (5), $\xi$ and $\lambda$ in Eqs. (28) and (29), $\Delta L$ in Eq. (30) are needed. The utilization of these experimentally fitted parameters could mislead understanding of the related physical nature since they actually consist of several factors.

In terms of this model, the phase stability of FM nanocrystals is strongly dependent on $C N$ reduction in the perpendicular direction of ultrathin films [97,98]. The different broken translational symmetries with different facets and $C N$ bring out the change of $T_{\mathrm{c}}(D)$ with different extent in light of the classic broken-bond rule $[129,130]$. This is the case of $T_{\mathrm{c}}(D)$ function of Fe nanocrystals shown in Fig. (4a) [93-98]. It should be noted that although $T_{\mathrm{c}}(\infty)$ [123] and $S_{\mathrm{m}}(\infty)$ [48] values of alloys are roughly estimated by their algebraic sum of elements, however, Fig. (4b) still presents Eq. (36) corresponding to experimental evidence well. This interesting result shows that for these alloys, algebria mean value has little error in comparison with the real value due to the similarity of elements consisting the alloys.

Fig. (4c) compares $T_{\mathrm{c}}(D)$ function of $\mathrm{Ni}$ nanocrystals with different $d$ between the experimental results and Eq. (36). $T_{\mathrm{c}}(D)$ decreases with $D$ and the decrease is stronger as $d$ drops. Since the freestanding crystals have larger surface/volume ratio, their size dependences are stronger than that of crystals supported by substrates. However, this dimension effect has been neglected in Eqs. (28)-(31).

Besides the aforementioned metals, $T_{\mathrm{c}}(D)$ functions of nanooxides of $\mathrm{Fe}_{3} \mathrm{O}_{4}$ [122] and $\mathrm{MnFe}_{2} \mathrm{O}_{4}$ [114] are given in Fig. (4d). The change tendencies are the same.

Table 2. The Parameters Utilized in the Calculations of Eq. (36) for FM Materials $\left(T_{\mathrm{c}}(\infty)\right.$ is in $\mathrm{K}, S_{\mathrm{m}}(\infty)$ in $\mathrm{J} \cdot \mathrm{g}$ Atom $^{-1} \cdot \mathrm{K}^{-1}$, and $h$ is in $\mathbf{~ m}$ )

\begin{tabular}{|c|c|c|c|}
\hline & $T_{\mathrm{c}}(\infty)[123,124]$ & $S_{\mathrm{m}}(\infty)^{\mathrm{a}}$ & $h(\mathbf{n m})^{\mathrm{d}}$ \\
\hline $\mathrm{Fe}$ & 1043 & 7.628 & 0.2483 \\
\hline Co & 1404 & 9.157 & 0.2497 \\
\hline $\mathrm{Ni}$ & 630 & 10.12 & 0.2492 \\
\hline $\mathrm{Gd}$ & 289 & 6.341 & 0.3575 \\
\hline $\mathrm{Tb}$ & 230 & 6.626 & 0.3525 \\
\hline $\mathrm{CoNi}$ & 1018 & $9.638^{b}$ & 0.2495 \\
\hline $\mathrm{Co}_{1} \mathrm{Ni}_{3}$ & 824.3 & $9.879^{\mathrm{b}}$ & 0.2493 \\
\hline $\mathrm{Co}_{1} \mathrm{Ni}_{9}$ & 708.3 & $10.02^{b}$ & 0.2493 \\
\hline $\mathrm{Fe}_{3} \mathrm{O}_{4}$ & 860 & 10.55 & 0.2220 \\
\hline $\mathrm{MnFe}_{2} \mathrm{O}_{4}$ & $573[114]$ & $10.55^{\mathrm{c}}$ & 0.2223 \\
\hline \multicolumn{4}{|c|}{ 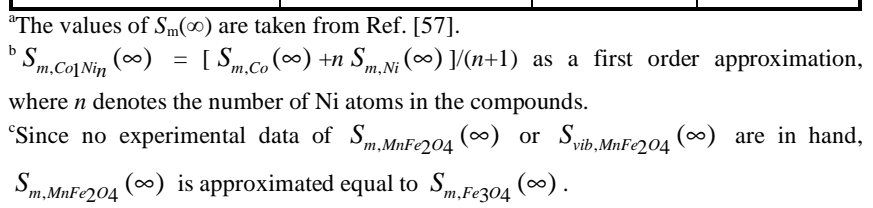 } \\
\hline
\end{tabular}

Fig. (5) shows $T_{\mathrm{c}}(D)$ functions of $\mathrm{Gd}$ and $\mathrm{Tb}$ rare earth nanocrystals between Eq. (36) and the corresponding experimental data. The wide distribution of $T_{\mathrm{c}}(D)$ of $\mathrm{Gd}$ nanocrystals indicates that the film morphology [108] and the lattice misfit at Gd film/substrate interface [105] affect $T_{\mathrm{c}}(D)$ of $\mathrm{Gd}$, which have been neglected in this model for simplicity.

Considering again the relationship of $\exp (-x) \approx 1-x$ when $x$ is small enough, under the condition that $D>>D_{0}$, Eq. (36) can be simplified as,

$$
T_{\mathrm{c}}(D) / T_{\mathrm{c}}(\infty) \approx 1-\left(\alpha_{\mathrm{s}}+\alpha_{\mathrm{i}}-2\right) D_{0} /(2 D)
$$

Comparing Eq. (37) with the scaling law of Eq. (28) for thin films with $D>\xi, T_{\mathrm{c}}(D)$ follows a power law curve with $\lambda=1$ [121]. Thus, Eq. (28) can be rewritten as $T_{\mathrm{c}}(D) / T_{\mathrm{c}}(\infty)=$ $1-[(\xi+h) /(2 D)][121]$. Associated with this relationship and Eq. (37), $\xi=\left(\alpha_{\mathrm{s}}+\alpha_{\mathrm{i}}-2\right) D_{0}-h$. For Fe, Co, Ni thin films, $\xi=$ $0.4048,0.5531,0.6322 \mathrm{~nm}$, respectively, which are approximately consistent with experimental and theoretical values of $0.4583,0.3962,0.7048 \mathrm{~nm}[121]$.

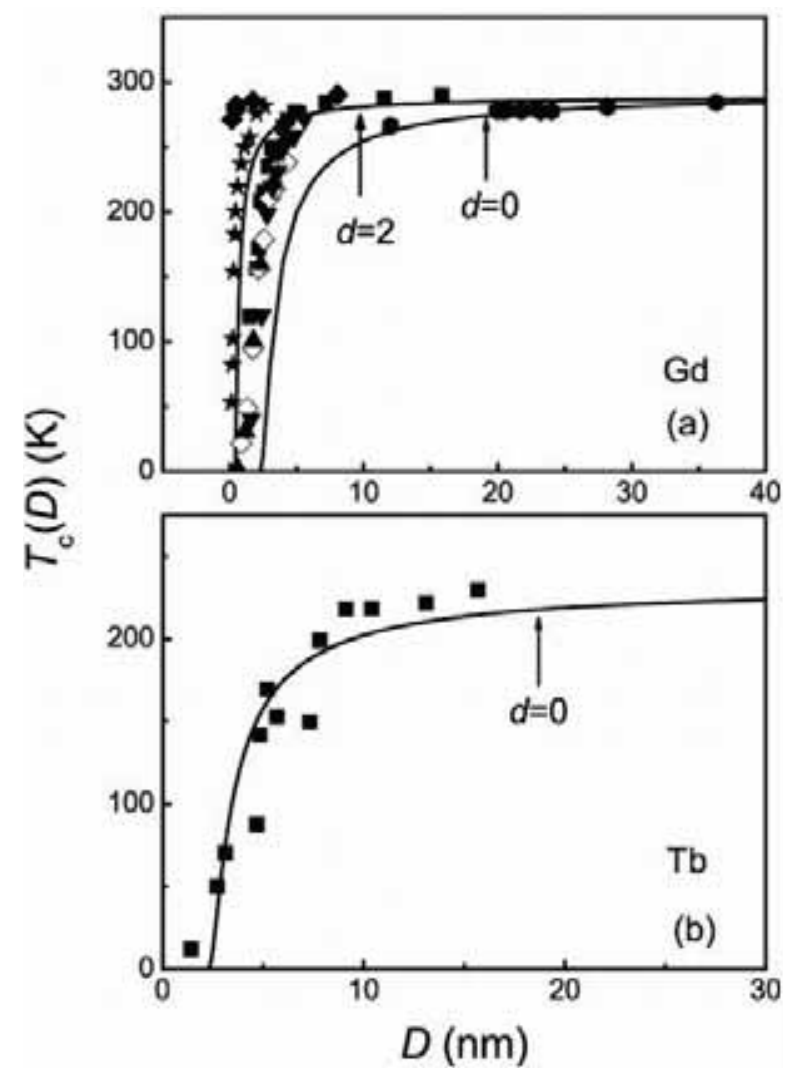

Fig. (5). Comparisons of $T_{\mathrm{c}}(D)$ functions between the model predictions in terms of Eq. (36) and available experimental results for (a) Gd nanoparticles and thin films, and (b) Tb nanoparticles. The necessary parameters: (a) $D_{0}=2.145,0.3575 \mathrm{~nm}$ according to Eq. (8) with $d=0,2$ for Gd nanoparticles and thin films, respectively, and $\alpha_{\mathrm{s}}=1.508$ in terms of Eq. (11), $\alpha_{\mathrm{i}}=\alpha_{\mathrm{s}}=1.508$ in terms of Eq. (35) with $J_{\mathrm{s}} \approx J_{\mathrm{i}}$ and $J_{\text {sub }} \approx 0$ for epitaxial films on magnetic inert substrates, the symbols $\boldsymbol{\square}$ [105], $\diamond$ [105], $\diamond[106], \boldsymbol{\nabla}$ [107], $\boldsymbol{\Delta}$ [107], $\star$ [108] denote the experimental measurements of $\mathrm{Gd} / \mathrm{W}(110)$, $\mathrm{Gd} / \mathrm{W}, \mathrm{Nb} / \mathrm{Gd}$ and $\mathrm{Gd} / \mathrm{Y}(0001)$ epitaxial films; [112] denotes $\mathrm{Gd}$ nanoparticles; (b) $D_{0}=2.115 \mathrm{~nm}$ according to Eq. (8) with $d=0$ for Tb nanoparticles, and $\alpha_{\mathrm{s}}=1.531$ in terms of Eq. (11), The symbol $\mathbf{a}$ [131] denotes $\mathrm{Tb}$ nanoparticles. Other parameters used to calculations are listed in Table $\mathbf{2}$ 
Eq. (37) has a similar form of Eq. (30) for nanoparticles. Combining Eqs. (30) and (37), $\Delta L \approx 2\left(\alpha_{\mathrm{s}}-1\right) D_{0} / 3$, or $\Delta L$ is related to $S_{\text {vib }}$ and $h$. Substituting these parameters into this equation, $\Delta L=0.8084,0.9692,0.7521,0.7512$ and $1.541 \mathrm{~nm}$ for $\mathrm{Ni}, \mathrm{Gd}, \mathrm{MnFe}_{2} \mathrm{O}_{4}, \mathrm{Fe}_{3} \mathrm{O}_{4}$ and $\mathrm{CuO}$ nanoparticles, respectively, namely, $\Delta L \approx 3 h$. Thus, when $D$ is larger than several nanometers, Eq. (30) can be rewritten as $T_{\mathrm{c}}(D) / T_{\mathrm{c}}(\infty) \approx 1$ $(9 h) /(2 D)$, which becomes a pure geometrical equation and emphasizes the surface contribution on $T_{\mathrm{c}}(D)$ function. Note that when Eqs. (5) and (37) are compared, $\sum_{i \leq 3} n_{i}\left(z_{s v} q_{s}^{-w}-1\right)$ $\approx-\left(\alpha_{\mathrm{s}}+\alpha_{\mathrm{i}}-2\right)$. Obviously, $w$ is a function of surface/interface conditions, which has been taken as an adjustable constant in Eq. (5).

Compared with the previous models [113,121-123], this unified model without any adjustable parameter can be utilized to predict the effects of dimension and interface on $T_{\mathrm{c}}(D)$ function through introducing the parameter $D_{0}$ and $\alpha$, respectively. When $0<\alpha<1, T_{\mathrm{c}}(D)$ increases with decreasing $D$, while contrary occurs when $\alpha>1$, which is determined by the common effects of both surface and film/substrate interface with different interface interaction strengths. Furthermore, when Eq. (37) is reasonable, the adjustable parameters appeared in Eqs. (28)-(30) and (5) could be quantitatively determined with more exact physical meaning.

\subsection{Size Dependent Magnetic Properties of FM Nanoc- rystals}

\subsubsection{Magnetization}

Magnetization is a property of magnets that describes to what extent they are affected by external magnetic field, which causes the spins within a material to align with the field. The maximum value of the magnetization in this state is called the saturation magnetization $M_{\mathrm{s}}$. As the magnitude of the magnetic field decreases, spins cease to be aligned with the field, and the total magnetization decreases. In ferromagnets, a residual magnetic moment remains at zero field. The corresponding value is called the remanent magnetization $M_{\mathrm{r}}$.

Based on the molecular field, the $T$-dependent spontaneous magnetization can be approximated in terms of the Brillouin function $B_{\mathrm{s}}(y)$ [88],

$$
M(T)=N g S \mu_{\mathrm{B}} B_{\mathrm{s}}(y)
$$

where $B_{\mathrm{s}}(y)=\frac{2 S+1}{2 S} \operatorname{coth}\left(\frac{2 S y+y}{2 S}\right)-\frac{1}{2 S} \operatorname{coth}\left(\frac{y}{2 S}\right)$, and $y=$ $2 g S \mu_{\mathrm{B}} \mu_{0} F_{\mathrm{m}} / k_{\mathrm{B}} T$, where $N$ is the number of molecules per unit volume, and $g$ is the Landé spectroscopic $g$-factor, $S$ is the spin quantum number of the atom, $\mu_{\mathrm{B}}$ is the Bohr magneton, $F_{\mathrm{m}}$ is the molecular field. If $T \leq 0.8 T_{\mathrm{c}}, M_{\mathrm{s}}(\infty, T) \approx M(\infty, T)$. As $T \rightarrow 0$, Eq. (38) can be simplified as [88],

$$
M(\infty, T \rightarrow 0)=M_{\mathrm{s}}(\infty, T \rightarrow 0)=N g S(\infty) \mu_{\mathrm{B}}
$$

with $B_{\mathrm{s}}(y) \approx 1$. If the orbital angular momentum is completely quenched, the total effective angular momentum of the atom is specified by $S$, and $g=2[85,88]$.
When $D$ drops, $M_{\mathrm{s}}(D, T \rightarrow 0)$ of FM nanocrystals varies dramatically. Generally, $M_{\mathrm{s}}(D, T \rightarrow 0)$ increases with quantized features at low $T$ [132-134] for FM metals, particularly of the $3 \mathrm{~d}$ series, which have itinerant moments and the magnitude of the moment is affected by details of the band structure. Pauling [135] and Goldschmidt [136] premises that $h$ would shrink by $12 \%, 4 \%$ and $3 \%$ if the $C N$ of an atom reduces from 12 to 4,6 and 8 , respectively, which causes the $3 \mathrm{~d}$ electrons to be more localized, and hence produces larger magnetic moment per atom than bulk. For instance, compared with the $\mathrm{Ni}$, Co and Fe bulk moments of $0.6 \mu_{\mathrm{B}}, 1.7 \mu_{\mathrm{B}}$ and $2.2 \mu_{\mathrm{B}}$ [88], respectively, the average magnetic moment per atom (the $M_{\mathrm{s}}$ per atom) increases to $1.05 \mu_{\mathrm{B}}, 2.35 \mu_{\mathrm{B}}$ and $3.1 \mu_{\mathrm{B}}$ as the cluster size is decreased from 700 atoms to 30 atoms [137]. $M_{\mathrm{s}}$ of a $1.8 \mathrm{~nm}$ sized Co particles is about $30 \%$ higher than that of the bulk [132]. $M_{\mathrm{s}}$ of Fe-Ni alloy films at $77<T<570 \mathrm{~K}$ increases gradually when $D$ is dropped from 75 to $35 \mathrm{~nm}$ [138]. In a Stern-Gerlach experiment conducted at $22 \mathrm{~K}, M_{\mathrm{s}}$ of freestanding $\mathrm{Fe}$ nanoclusters containing 2-17 atoms to be larger than that of the bulk counterparts [133].

Furthermore, the rather abrupt termination at the surface also causes quantum mechanical electronic shock waves to propagate into particles or thin films in the form of spatially oscillating charge- and spin-density waves [134,137]. If the localization probability is proportional to $\varepsilon$, then the densely localized electrons contribute to $S$ [134]. Using a shell structure in BOLS correlation that calculates the magnetic moment of every atom layer-by-layer leads to $M_{\mathrm{s}}(D, T)$ at very low $T$ [134],

$$
\left.\frac{M_{s}(D, T)}{M_{s}(\infty, T)}\right|_{T \rightarrow 0}=1+\sum_{i \leq 3} h_{i j}\left(q_{i}^{-w}-1\right)
$$

According to Eq. (40), $M_{\mathrm{s}}(D, T)$ functions of nanocrystals is presented in Fig. (6). Agreement with computer simulation results is found. This is also confirmed by the calculations based on the tight binding approximation [139].

In contrast, ionic compounds are characterized by spatially localized valence electrons. Their distribution in atomic-like orbitals in accordance with Hund's rules results in well-defined, localized moments on each magnetic ion. Thus, at low $T$, the moment of each ion is less sensitive to the proximity of a surface [140]. However, due to imperfection of $C N$ of surface ions, the orientation of each moment can be altered, which results in a disordered spin configuration near the surface and a reduced net moment as compared to bulk materials [141-143].

Whereas $M_{\mathrm{s}}\left(D, T_{\mathrm{a}}\right)$ of FM nanocrystals decreases with dropping $D$, remarkable reductions of $M_{\mathrm{s}}\left(D, T_{\mathrm{a}}\right)$ of Fe-Ni invar alloy $(<40 \mathrm{~nm})$ [144], Ni thin films [145,146] and Fe, $\mathrm{Co}$ and $\mathrm{Ni}$ nanoparticles [147] have been found. In addition, $M_{\mathrm{s}}\left(D, T_{\mathrm{a}}\right)$ of $\gamma-\mathrm{Fe}_{2} \mathrm{O}_{3}$ [148], $\mathrm{Fe}_{3} \mathrm{O}_{4}$ [149-151], $\mathrm{CoFe}_{2} \mathrm{O}_{4}$ [152,153] and $\mathrm{MnFe}_{2} \mathrm{O}_{4}$ [154,155] ferromagnetic oxide nanoparticles have the same tendency with $D$. To clarify this phenomenon, Coey et al. firstly proposed a random canting of the particles surface spins that is caused by competing antiferromagnetic exchange interactions at the surface [156]. 
Since then, the problem has been revisited with arguments in favor of a surface origin [157] and in favor of a finite size effect [158]. However, no clear conclusions about it have been given yet [159]. Recently, Kodama et al. proposed a disordered surface spin structure model to illustrate the low $M_{\mathrm{s}}$ of $\mathrm{NiFe}_{2} \mathrm{O}_{4}$ nanoparticles [160], while Mamiya et al. attributed the low $M_{\mathrm{s}}$ of $\varepsilon-\mathrm{Fe}_{3} \mathrm{~N}$ nanoparticles to dipolar interparticle interactions [161]. When a core-shell structure is assumed, where the shell layer (non-magnetic layer) has a fitting thickness $t_{0}$ with lower $M_{\mathrm{s}}$ than the corresponding bulk one [114], Tang et al. derived an empirical relationship [114],

$$
M_{\mathrm{s}}\left(D, T_{\mathrm{a}}\right) / M_{\mathrm{s}}\left(\infty, T_{\mathrm{a}}\right)=1-6 t_{0} / D
$$

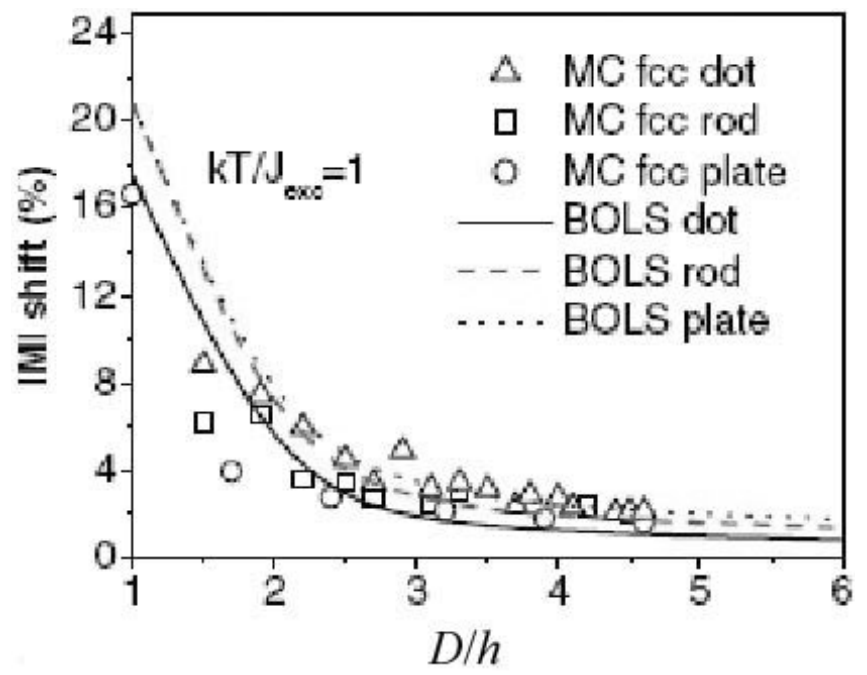

Fig. (6). $M_{\mathrm{s}}\left(D, T_{\mathrm{a}}\right)$ function at $k_{\mathrm{B}} T / J_{\mathrm{exc}}=1$ [134]. (Reproduced with permission from Ref. [134]. Copyright 2005, Elsevier).

Recently, $M_{\mathrm{s}}(D)$ is related to $T_{\mathrm{c}}(D)$ by incorporating BOLS correlation mechanism into the Ising model and the Brillouin function with $\varepsilon(S, T) \approx 4$ for FM crystals at $T_{\mathrm{a}}$ $[134,162], \Delta M_{\mathrm{s}}(D, T) / M_{\mathrm{s}}(\infty, T)=\varepsilon(S, T) \sum_{i \leq 3} n_{i}\left(z_{i b} q_{i}^{-w}-1\right)$. In reality, the reduced $D$ results in enhancement of $S$ value due to the contribution from the charge localization, which suggests taking responsibility of $M_{\mathrm{s}}(D)$ enhancement at very low $T[134,162]$, as the aforesaid. However, the size effect on $S$ becomes insignificant compared with that of $E_{\mathrm{exc}}$ at $T_{\mathrm{a}}$ where $E_{\text {exc }}$ dominates the magnetic behavior. Thus, $\varepsilon\left(S, T_{\mathrm{a}}\right) \approx 4$ as a constant is taken. If $F_{\mathrm{m}} \propto E_{\text {exc }}$ or $F_{\mathrm{m}}=p E_{\text {exc }}$, Eq. (38) can be rewritten as $M_{\mathrm{s}}(T)=$

$N g S \mu_{\mathrm{B}}\left\{\frac{2 S+1}{2 S} \operatorname{coth}\left[\frac{(2 S+1) g \mu_{B}}{2 k T} p E_{e x c}\right]-\frac{1}{2 S} \operatorname{coth}\left(\frac{g \mu_{B}}{2 S} p E_{e x c}\right)\right\}$. Differentiating it against $E_{\text {exc }}(D)$ leads to $M_{\mathrm{s}}(D, T)$ in the mid $T$ region $[134,162]$,

$$
\frac{M_{s}(D, T)}{M_{s}(\infty, T)}=\varepsilon(S, T) \frac{E_{e x c}(D)}{E_{e x c}(\infty)}
$$

In terms of Eqs. (34) and (42), it reads,

$$
M_{\mathrm{s}}\left(D, T_{\mathrm{a}}\right) / M_{\mathrm{s}}\left(\infty, T_{\mathrm{a}}\right)=4 \exp \left[-(\alpha-1) /\left(D / D_{0^{-}}-1\right)\right]-3
$$

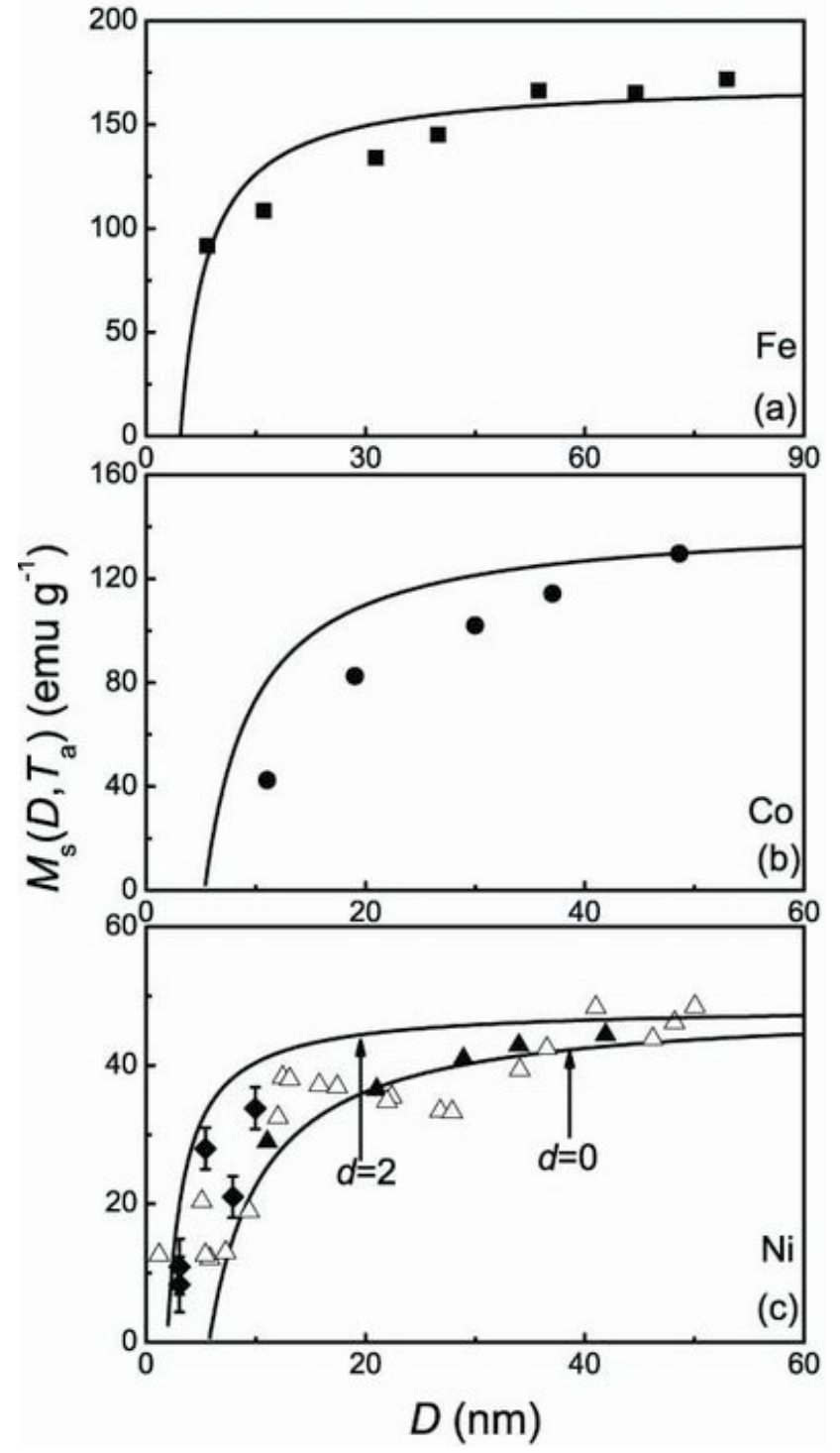

Fig. (7). Comparisons of $M_{\mathrm{s}}\left(D, T_{\mathrm{a}}\right)$ function between model predictions according to Eq. (43) and available experimental results for (a) Fe nanoparticles (ם [147]), (b) Co nanoparticles ( [147]), (c) Ni nanoparticles ( $\boldsymbol{\Delta}[147])$ and thin films $(\diamond[145]$ and $\triangle[146])$. For Fe, Co and Ni nanoparticles, $\alpha_{s}=1.612,1.734,1.811$ in terms of Eq. (11), $D_{0}=1.489,1.504,1.495 \mathrm{~nm}$ according to Eq. (8) with $d$ $=0$; For Ni films deposited on glass substrates, $\alpha_{\mathrm{i}}=\alpha_{\mathrm{s}}=1.811$ in light of Eq. (35) with $J_{\mathrm{s}} \approx J_{\mathrm{i}}$ and $J_{\text {sub }} \approx 0$, and $D_{0}=(2 h) / 2=0.2492$ $\mathrm{nm}$ in terms of Eq. (8) with $d=2$. The necessary parameters utilized in the calculations are listed in Table $\mathbf{3}$.

Fig. (7a-c) show the comparisons of $M_{\mathrm{s}}\left(D, T_{\mathrm{a}}\right)$ functions between Eq. (43) and available experimental results for $\mathrm{Fe}$, $\mathrm{Co}$, Ni nanocrystals (nanoparticles and thin films), where the necessary parameters utilized in the calculations are listed in Table 3.

As shown in this figure, $M_{\mathrm{s}}\left(D, T_{\mathrm{a}}\right)$ decreases as $D$ and $d$ is reduced. For Co nanoparticles, error is big when $D<30 \mathrm{~nm}$ and the reason may be related to the structural transformation from the bulk hcp structure to a fcc one in nanometer size [146,147]. 
Table 3. The Necessary Parameters Employed in Eq. (43) $\left(M_{\mathrm{s}}\left(\infty, T_{\mathrm{a}}\right)\right.$ is in emu $\cdot \mathrm{g}^{-1}, S_{\text {vib }}$ and $h$ in $\mathrm{J} \cdot \mathrm{g}-\mathrm{Atom}^{-1} \cdot \mathrm{K}^{-1}$ and $\mathrm{nm}$, Respectively)

\begin{tabular}{|c|c|c|c|}
\hline & $\boldsymbol{M}_{\mathbf{s}}\left(\boldsymbol{\infty}, \boldsymbol{T}_{\mathbf{a}}\right)[\mathbf{1 4 6}]$ & $\boldsymbol{S}_{\text {vib }}(\boldsymbol{\infty})$ & $\boldsymbol{h}[\mathbf{1 4 6 ]}$ \\
\hline \hline $\mathrm{Fe}$ & 171.0 & 7.628 & 0.2482 \\
\hline $\mathrm{Co}$ & 143.0 & 9.157 & 0.2506 \\
\hline $\mathrm{Ni}$ & 48.5 & 10.12 & 0.2492 \\
\hline$\gamma-\mathrm{Fe}_{2} \mathrm{O}_{3}$ & 76.0 & 5.704 & 0.1850 \\
\hline $\mathrm{Fe}_{3} \mathrm{O}_{4}$ & 92.0 & 10.55 & 0.1890 \\
\hline $\mathrm{MnFe}_{2} \mathrm{O}_{4}$ & 80.0 & 10.55 & 0.2293 \\
\hline $\mathrm{CoFe}_{2} \mathrm{O}_{4}$ & 75.0 & 10.55 & 0.2264 \\
\hline
\end{tabular}

Similar results can be obtained for $M_{\mathrm{s}}\left(D, T_{\mathrm{a}}\right)$ of ferrimagnetic $\gamma-\mathrm{Fe}_{2} \mathrm{O}_{3}$ [148], $\mathrm{Fe}_{3} \mathrm{O}_{4}$ [14-151], $\mathrm{CoFe}_{2} \mathrm{O}_{4}$ [152,153] and $\mathrm{MnFe}_{2} \mathrm{O}_{4}[114,154,155]$ nanooxides, of which the comparisons between model predictions of Eq. (43) and available experimental evidence are shown in Fig. (8).

Using $\exp (-x) \approx 1-x$ into Eq. $(43), M_{\mathrm{s}}\left(D, T_{\mathrm{a}}\right) / M_{\mathrm{s}}\left(\infty, T_{\mathrm{a}}\right) \approx$ $1-8 S_{\text {vib }}(\infty) D_{0} /(3 R D)$. Let the equation be equal to zero, or $M_{\mathrm{s}}\left(t_{0}, T_{\mathrm{a}}\right)=0$, there is,

$$
t_{0} \approx 8 S_{\mathrm{vib}}(\infty) D_{0} /(3 R)
$$

Taking the related data into Eq. (44), $t_{0} \approx 2.075$ and $t_{0} \approx$ $4.4 \mathrm{~nm}$ for $\gamma-\mathrm{Fe}_{2} \mathrm{O}_{3}$ and $\mathrm{MnFe}_{2} \mathrm{O}_{4}$ nanoparticles, respectively, which are approximately consistent with $t_{0}=3.42 \mathrm{~nm}$ for $\gamma$ $\mathrm{Fe}_{2} \mathrm{O}_{3}$ and $t_{0}=3.60 \mathrm{~nm}$ for $\mathrm{MnFe}_{2} \mathrm{O}_{4}$ determined by Eq. (41).

In terms of Eqs. (34) and (42), there is,

$$
M_{\mathrm{s}}\left(D, T_{\mathrm{a}}\right) / M_{\mathrm{s}}\left(\infty, T_{\mathrm{a}}\right)=4 T_{\mathrm{c}}(D) / T_{\mathrm{c}}(\infty)-3
$$

Eq. (45) indicates that the suppression of $M_{\mathrm{s}}\left(D, T_{\mathrm{a}}\right)$ is about four times of that of $T_{\mathrm{c}}(D)$. This can be qualitatively explained as the following: On one hand, the absolute value of $E_{\text {exc }}(D)$ drops due to the increase of surface atoms, which leads to the weakening of inter-spin interaction and thus the suppression of $M_{\mathrm{s}}\left(D, T_{\mathrm{a}}\right)$ [146]; On the other hand, with rising $T$, increased thermal vibrations tend to counteract the dipole coupling forces. Consequently, $M_{\mathrm{s}}$ gradually diminishes following the temperature dependent form of $\left(T_{\mathrm{c}} / T-1\right)^{1 / \lambda}$ [120]. Because $T_{\mathrm{c}}$ decreases with $D$ while $T=T_{\mathrm{a}}$, both of $T_{\mathrm{c}} / T$ and $M_{\mathrm{s}}$ reduce. In other words, the effect of decreasing $D$ is equivalent to that of rising $T$. Thus, the both effects bring out stronger suppression of $M_{\mathrm{s}}\left(D, T_{\mathrm{a}}\right)$ than that of $T_{\mathrm{c}}(D)$ at $T_{\mathrm{a}}$ where the latter is only induced by reducing $D$ [146].

\subsubsection{Anisotropy}

Basically, the two main sources of the magnetic anisotropy are the magnetic dipolar interaction and the spin-orbit interaction. The former is controllable by altering the shape of nanocrystals, namely the shape anisotropy. This is of particular importance in thin films, and is largely responsible for the in-plane magnetization. The latter gives rise to the crystalline anisotropy, and favors energetically alignment of the magnetization along the easy axis direction. In hcp Co, this is $c$ axis, while in cubic systems such as $\mathrm{Fe}$ and $\mathrm{Ni}$, easy axes

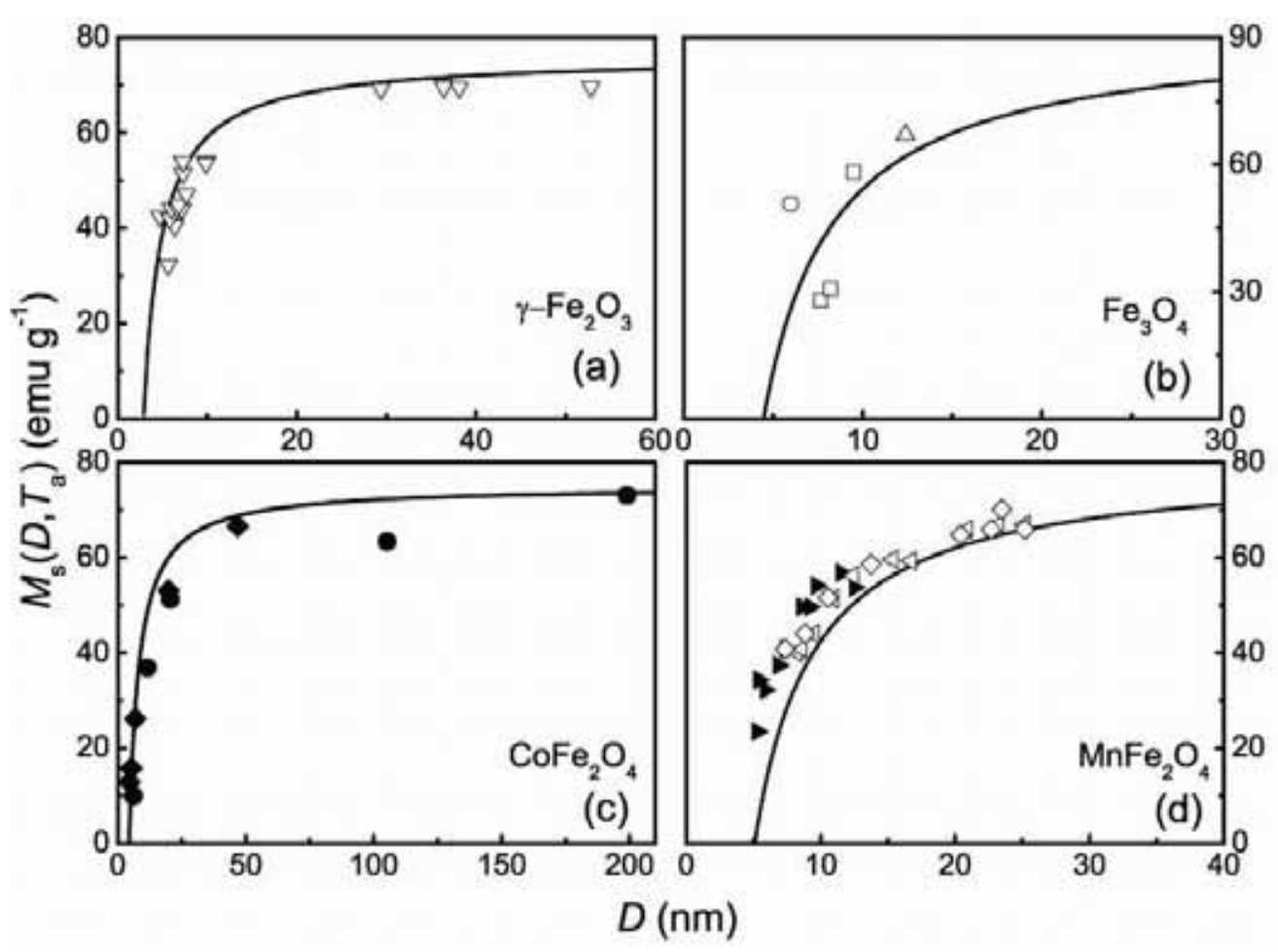

Fig. (8). Comparisons of $M_{\mathrm{s}}\left(D, T_{\mathrm{a}}\right)$ functions between model predictions according to Eq. (43) and available experimental evidence: (a) $\gamma$ -

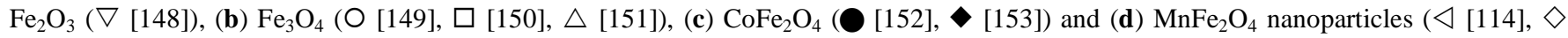
[154], [155]). The parameters: $\alpha_{\mathrm{s}}=1.457,1.846,1.846,1.846$ in terms of Eq. (11) and $D_{0}=1.11,1.134,1.376$, and 1.358 in terms of Eq. (8) with $d=0$ for $\gamma-\mathrm{Fe}_{2} \mathrm{O}_{3}, \mathrm{Fe}_{3} \mathrm{O}_{4}, \mathrm{MnFe}_{2} \mathrm{O}_{4}$ and $\mathrm{CoFe}_{2} \mathrm{O}_{4}$ nanoparticles, respectively. The necessary parameters are listed in Table 3. 
are $\langle 100\rangle$ and $\langle 111\rangle$, respectively. For FM nanocrystals, surface or interface atoms have narrowing local density of state at the Fermi level, and thus modifie the surface magnetic anisotropy [28]. The phenomenological description of the effective magnetic anisotropy $K(D)$ is given by Néel model [92,163],

$$
K(D)=K_{\mathrm{v}}+K_{\mathrm{i}}+K_{\mathrm{s}} / D
$$

where only the surface contribution is assumed to be sizedependent. This relationship represents a weighted average of the magnetic anisotropy energy of interface (surface) atoms and the inner atoms of magnetic layer with size of $D$.

$K_{\mathrm{i}}=-3 \lambda_{\mathrm{m}} E_{\mathrm{m}} \varepsilon_{\mathrm{m}} / 2$ is the strain anisotropy induced by the lattice mismatch $\left[\eta=\left(h-h_{\text {sub }}\right) / h\right]$ at film/substrate interface where $\lambda_{\mathrm{m}}$ is the magnetostriction constant depending on the orientation and being able to be positive or negative, $\varepsilon_{\mathrm{m}}$ and $E_{\mathrm{m}}$ are the strain and the elastic modulus, respectively. In terms of the van der Merwe model, in which elastic as well as dislocation energies are considered [163,164], two regimes should be distinguished: the coherent $\left(D<D_{\mathrm{c}}\right)$ and incoherent $\left(D>D_{\mathrm{c}}\right)$ regimes, where $D_{\mathrm{c}}$ denotes a film thickness at which a transition from the stained structure to a normal one occurs [163].

For the FM/substrates in the coherent regime, $\varepsilon_{\mathrm{m}}=-\eta$ since the substrates is of infinite thickness [163], which is independent of $D$ and contributes only to $K_{\mathrm{v}}$ [163]. The elastic energy $U_{\mathrm{e}}$ associated with the coherent interface is proportional to the strained volume. Increasing $D$ will therefore increase $U_{\mathrm{e}}$. At $D_{\mathrm{c}}$, it becomes energetically more favorable to introduce misfit dislocations, which partially accommodate the lattice misfit, allowing the uniform strain to be reduced [163,165]. When $D>D_{\mathrm{c}}, \varepsilon_{\mathrm{m}}$ has been given by $[163,166], \varepsilon_{\mathrm{m}}=-\eta D_{\mathrm{c}} / D$, where $D_{\mathrm{c}}$ is determined by the energy balance between $U_{\mathrm{e}}$ and film/substrate interface energy $U_{\mathrm{i}}[165]$, namely, $U_{\mathrm{e}}-U_{\mathrm{i}}=0$, where $U_{\mathrm{e}}=V_{\mathrm{m}} E\left(a-a_{\mathrm{sub}}\right)^{2} /\left(3-3 v_{\mathrm{a}}\right)$, and $U_{\mathrm{i}}=\gamma_{\mathrm{i}} V_{\mathrm{m}} /(D \sin \theta)$ with $v_{\mathrm{a}}$ being the Poisson ratio and $\gamma_{\mathrm{i}}=$ $4 \bar{h}_{v i b} \bar{H}_{m} /\left(3 \bar{V}_{m} R\right)$ where the upper bar denotes the corresponding mean quantities of films and substrates, respectively. Therefore [165], $D_{\mathrm{c}}=\frac{4\left(1-v_{a}\right) \bar{S}_{v i b} \bar{H}_{m} h}{\sin \theta \bar{V}_{m} R E\left(a_{m}-a_{s u b}\right)^{2}}$. Combining above relationships leads to,

$$
K_{\mathrm{i}}=\frac{6 \lambda_{m} E_{m}\left(1-v_{a}\right) \bar{S}_{v i b} \bar{H}_{m} h \eta}{\sin \theta \bar{V}_{m} R E\left(a-a_{s u b}\right)^{2} D}
$$

which follows the common analysis of anisotropy data as introduced by Eq. (46).

When films have the same two surfaces or interfaces, Eq. (46) is rewritten as, $K(D)=K_{\mathrm{v}}+2 K_{\mathrm{s}} / D$, or $K(D)=K_{\mathrm{v}}+2 K_{\mathrm{i}} / D$ [167-171].

Surface steps of films also lead to magnetic anisotropy since the crystallographic symmetry at the surface is broken not only perpendicular to but also parallel to the film-plane. This contribution is called the step-induced magnetic anisotropy $K_{\text {step }}$ [28], which is characterized by a change in the shape of the magnetization curve. This effect has been experimentally observed, such as the systems of $\mathrm{Ni} / \mathrm{Fe} / \mathrm{Ag}(001)$
[172], $\mathrm{Fe} / \mathrm{Ag}(001) \quad[173,174], \mathrm{Fe} / \mathrm{W}(001) \quad[175,176], \mathrm{Co} / \mathrm{Cu}$ (1113) [177].

In terms of the Néel model, Chuang et al. [178] added this term into Eq. (46),

$$
K(D)=K(\infty)-2 K_{\mathrm{s}} / D-2 K_{\text {step }} / D t_{\mathrm{w}}
$$

where $t_{\mathrm{w}}$ is the terrace width, $K_{\text {step }}=K_{\text {step,edge }}+K_{\text {step,corner }}$ since edge and corner of steps ( $K_{\text {step,edge }}$ and $\left.K_{\text {step,corner }}\right)$ contribute $K_{\text {step }}$, respectively. This model properly accounts for the observed behaviors.

Magnetic anisotropies are also temperature-dependent [179,180], which vanish at $T_{\mathrm{c}}$ in particular for itinerant ferromagnets expect for $\mathrm{Gd}$, of which the finite anisotropies may be present at $T>T_{\mathrm{c}}$ [181]. The different size- and temperature-dependences of surface, volume and shape anisotropies may give rise to the spin reorientation transition (SRT) at the corresponding temperature $T_{\mathrm{r}}(D)$. In most cases, SRT refers to the variation of the polar angle, i.e., a rotation from a perpendicular to an in-plane direction of thin film magnetization or vice versa. This should be distinguished from the magnetic reversal due to the external magnetic field.

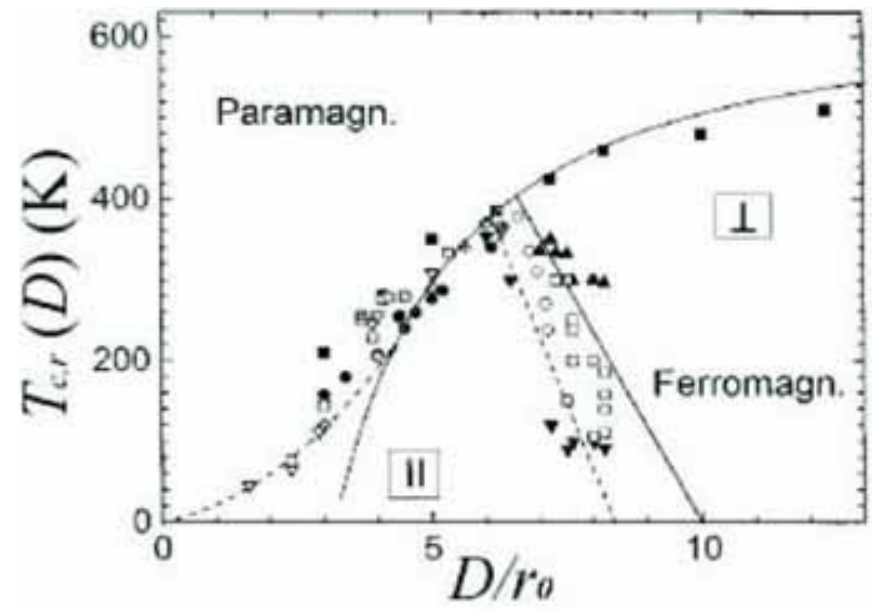

Fig. (9). Magnetic phase diagram for $\mathrm{Ni} / \mathrm{Cu}(001)$ as a function of $D$ [198]. The lines separate the paramagnetic, the ferromagnetic phase with the perpendicular, and the in-plane magnetization directions. The solid line represents the finite-size scaling, and the dashed line is a guide to the eyes to visualize that the films down below two monolayers. The symbols and correspond to the complete inplane and perpendicular ferromagnetic orientation, $\square$ and $\bigcirc$ correspond to $0^{\circ}<\theta<90^{\circ}$. (Reproduced with permission from Ref. [18]. Copyright 1997, AIP).

Since the first observation of SRT by Gradmann and Müller [182], a large number of related experimental investigations, such as $\mathrm{Fe} / \mathrm{Cu}(100)$ [183,184], $\mathrm{Fe} / \mathrm{Cu} / \mathrm{Si}(111)$ [185], $\mathrm{Fe} / \mathrm{Cu}(001) \quad[186,187], \mathrm{Fe} / \mathrm{Ag}(001) \quad[97,188], \mathrm{Fe} / \mathrm{Cr}(110)$ [167], $\mathrm{Fe} / \mathrm{Si}(111)$ [189], and $\mathrm{Co} / \mathrm{Au}(111)$ [190,191], $\mathrm{Co} / \mathrm{Pd}(111)$ [192], $\mathrm{Co} / \mathrm{Pt}(111)$ [192], $\mathrm{Co} / \mathrm{W}(110)$ [193] thin films showing the polar SRT, $m_{\perp} \rightarrow m_{\|}$, and $\mathrm{Ni} / \mathrm{Cu}(001)$ [194,195] exhibiting the reversed polar SRT $m_{\|} \rightarrow m_{\perp}$ with increasing $T$ and $D$, where $m_{\perp}$ and $m_{\|}$denote the perpendicular and in-plane magnetic directions. Calculations based on the mean-field and perturbation theory [196,197] have described successfully this SRT. Fig. (9) shows the typical 
phase diagram of $\mathrm{Ni} / \mathrm{Cu}(001)$ thin films system [198], where $T_{\mathrm{c}}(D)$ and $T_{\mathrm{r}}(D)$ functions are presented.

\subsubsection{Coercivity}

With reference to the saturation hysteresis cycle, the coercive force $H_{\mathrm{c}}$ is defined customarily as the value of the magnetizing field corresponding to the point $H=0 . H_{\mathrm{c}}$ is a sensitive property of ferromagnetic materials and provides an important criterion in the selection of these materials for practical use [186].

In order to reverse the direction of magnetization in a small particle, it is necessary that the magnetic energy acquired by the particle in the external magnetic field is greater than the internal energy tending to prevent ration of the domain direction. The latter is related to the effective internal anisotropy consisting of the magnetocrystals [199], shape [200] and strain anisotropies [201], which result in $H_{\mathrm{cm}}=$ $2 K(\infty) / M_{\mathrm{s}}(\infty), H_{\mathrm{cs}}=\left(N_{\mathrm{t}}-N_{0}\right) M_{\mathrm{s}}(\infty)$ and $H_{\mathrm{cst}}=3 \lambda_{\mathrm{m}} \sigma_{\mathrm{m}} / M_{\mathrm{s}}(\infty)$, respectively, where $N_{0}$ denotes the demagnetizing factor of the prolate spheroid in the direction of the major axis, and $N_{\mathrm{t}}$ denotes the demagnetizing factor in any direction at right angles to the long axis and $\sigma_{\mathrm{m}}$ is the applied stress [86]. $N_{\mathrm{t}}=$ $N_{0}=4 \pi / 3$ for a sphere.

A common trend of $H_{\mathrm{c}}(D)$ function is illustrated in Fig. (10) $[202,203] . H_{\mathrm{c}}(D) \propto D^{-1}$ down to the single-domain size $D_{\mathrm{s}}$, and then $H_{\mathrm{c}}(D)$ drops in a $D^{6}$ fashion. $D_{\mathrm{s}}$ has been estimated theoretically [199], for instance, $D_{\mathrm{s}}=14,70,55,128$, $166 \mathrm{~nm}$ for $\mathrm{Fe}, \mathrm{Co}, \mathrm{Ni}, \mathrm{Fe}_{3} \mathrm{O}_{4}$ and $\gamma-\mathrm{Fe}_{2} \mathrm{O}_{3}$, respectively [202]. In the region of $D>D_{\mathrm{s}}$, energetic considerations favor the formation of domain walls. The magnetization reversal thus occurs through the nucleation and motion of these walls [86]. $H_{\mathrm{c}}(D)$ of spherical particles is given by [86,204],

$$
H_{\mathrm{c}}(D) / H_{\mathrm{c}}(\infty)=1-D / d_{0}
$$

where $H_{\mathrm{c}}(\infty)=2 K(\infty) / M_{\mathrm{s}}(\infty), d_{0}=24\left[K(\infty) k_{\mathrm{B}} T_{\mathrm{c}}(\infty) / a\right]^{1 / 2} / M_{\mathrm{s}}{ }^{2}$ $(\infty)$. As $D$ is reduced, the formation of domain walls becomes energetically unfavorable, which results in the increasing energy to rotate spins. So does $H_{\mathrm{c}}(D)$ function [86,204,205].

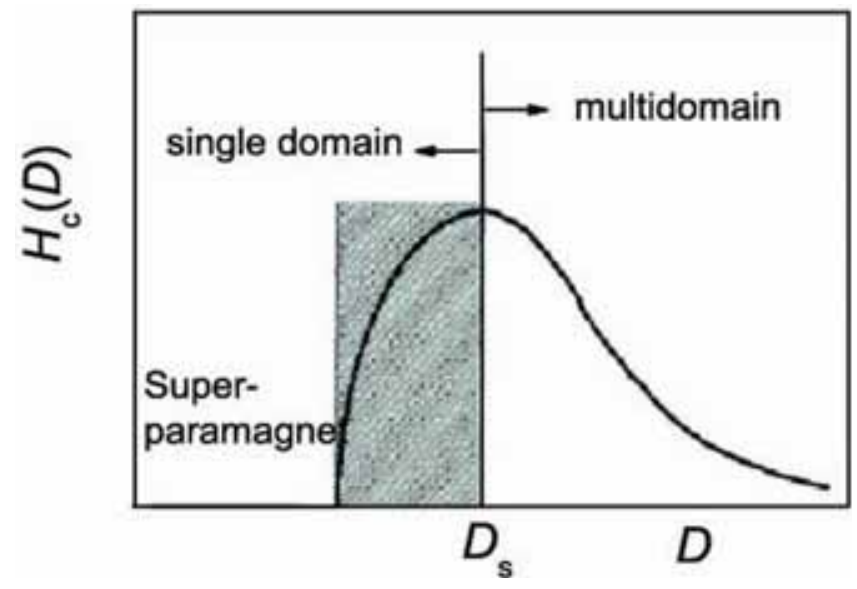

Fig. (10). Qualitative illustration of the $H_{\mathrm{c}}(D)$ function [202]. (Reproduced with permission from Ref. [202]. Copyright 1996, ACS).
A single domain particle is present when $D \leq D_{\text {s }}$, which results in the coherent rotation of spins of the whole particles rather than the motion of domain walls. For the magnetic granular solids consisting of nanometer-sized magnetic particles uniformly dispersed and embedded in an immiscible insulating or metallic medium at $T$ below the blocking temperature of superparamagnets $T_{\mathrm{b}}$ [206], the structure brings out randomness in interaction of the magnetic moments, including random isotropic exchange and random anisotropy. A reasonable amount of randomness in the isotropic exchange is not expected to qualitatively affect the magnetic behavior, even near the critical point. However, random anisotropy strongly changes magnetic properties. Most importantly, it breaks the rotational symmetry of the Hamiltonian and leads to hysteresis and even hard magnetic behavior in random amorphous magnets. Based on the random anisotropy theory [207], $H_{\mathrm{c}}(D)$ has been deduced as,

$$
H_{\mathrm{c}}(D) \approx K_{2}^{4} D^{6} /\left(20 A^{3} M_{0}\right)
$$

where $K_{2}$ is the strength of local uniaxial anisotropy and $M_{0}$ is the magnitude of the local magnetization vector. This model has experimentally been confirmed for Fe [203,208], Co [203], Ni [203], Fe/Co alloys [208], FeNbSiB, Fe$\mathrm{CuNbSiB}$ and FeCuVSiB [209], FeCoZr [210] alloys.

Recently, $H_{\mathrm{c}}(D)$ function of $\mathrm{Cu}_{80} \mathrm{Co}_{20}$ thin films is empirically proposed based on the mechanism of nucleation and motion of domain walls [211],

$$
H_{\mathrm{c}}(D)=2 E_{\mathrm{b}} /\left(\mu_{0} M_{\mathrm{s}}\right)-N_{\mathrm{eff}}(D) M_{\mathrm{S}}
$$

where $E_{\mathrm{b}}=E_{\mathrm{a}}+E_{\text {int }}$ is the anisotropy energy with $E_{\mathrm{a}}$ and $E_{\text {int }}$ being the anisotropy energy barrier and the interaction energy barrier, respectively, and $N_{\text {eff }}(D) \propto \tan ^{-1}\left[D /\left(2 D_{\mathrm{s}}{ }^{2}+D^{2}\right)^{1 / 2}\right]$ is the demagnetization factor [211].

As $D<L_{\mathrm{w}}$, the spins are increasingly affected by thermal fluctuations [202]. When $T$ is sufficiently high, the energy barrier $E_{\mathrm{a}}$ is overcome by the thermal energy [212-214]. The system becomes superparamagnetic, and $H_{\mathrm{c}}(D) \rightarrow 0$ because thermal fluctuations can prevent the existence of a stable magnetization.

\subsubsection{Superparamagnetism}

Superparamagnetic relaxation can be described by the Arrhenius relation of $\tau=\tau_{0} \exp \left[E_{\mathrm{a}} / k_{\mathrm{B}} T\right]$ where $\tau$ and $\tau_{0}$ are the relaxation and the characteristic time [202,206,215]. Because of this relaxation, $M_{\mathrm{r}}$ and $H_{\mathrm{c}}$ decrease with increasing $T$ and vanish at $T_{\mathrm{b}}=E_{\mathrm{a}} /\left[k_{\mathrm{B}} \ln \left(\tau / \tau_{0}\right)\right]$ [206,206213-215] with $E_{\mathrm{a}}=K V$, where $V$ is the volume of particles. At $T<T_{\mathrm{b}}$, the systems can show ferromagnetic nature, e.g. a hysteresis loop. However, the systems become paramagnetic or superparamagnetic state at $T>T_{\mathrm{b}}$, even though the magnetic moments within each particle remain ferromagnetically aligned [206]. It should be noted that the superparamagnetic relaxation is a kinetic process. Thus, $T_{\mathrm{b}}$ is a case value in different experimental measurements, such as magnetometery $(\tau=10$ s) [214], AC susceptibility ( $\left.\tau=10^{-4}-10^{-1} \mathrm{~s}\right)$ [216], Mössbauer spectroscopy $\left(\tau=10^{-8}\right.$ s) [214], small-angle neutron diffraction $\left(\tau=5 \times 10^{-12} \mathrm{~s}\right)$ [217], as well as low-field susceptibility in both zero-field-cooled and field-cooled modes [214], etc. 
If $\tau=100 \mathrm{~s}$ and $\tau_{0}=10^{-9} \mathrm{~s}, T_{\mathrm{b}}=E_{\mathrm{a}} /\left(25 k_{\mathrm{B}}\right)[202,218]$. Since $T_{\mathrm{b}}$ is a function of $E_{\mathrm{a}}$, it is that of $D$, as shown in experimental measurements for Co [219] clusters [220], $\mathrm{MgFe}_{2} \mathrm{O}_{4}$ [221], $\mathrm{CoFe}_{2} \mathrm{O}_{4}$ [221,222], $\mathrm{MnFe}_{2} \mathrm{O}_{4}$ [223,224], $\mathrm{CoCr}_{x} \mathrm{Fe}_{2-}$ ${ }_{x} \mathrm{O}_{4}$ [225] and Ni-Zn, Zn-Mn, Ni-Mn ferrites [226], which is reviewed in reference [227].

The temperature-dependent magnetization behavior of a system of particles $M_{\mathrm{sp}}(T)$ follows the Langevin function [215], $M_{\mathrm{sp}} / M_{\mathrm{p}}=L\left(M_{\mathrm{p}} H / k_{\mathrm{B}} T\right)=\operatorname{coth}\left(M_{\mathrm{p}} H / k_{\mathrm{B}} T\right)-k_{\mathrm{B}} T / M_{\mathrm{p}} H$, where $M_{\mathrm{p}}=M_{\mathrm{s}} V$ is the giant magnetic moment of a single particle. In suitable ranges of $T$ and $H$, the Langevin function depends sensitively on $M_{\mathrm{p}}$ and hence $D$ [206]. At low field $\left(M_{\mathrm{p}} H<<k_{\mathrm{B}} T\right)$, the magnetization behaves as $M_{\mathrm{p}} H /\left(3 k_{\mathrm{B}} T\right)$ and high fields $\left(M_{\mathrm{p}} H \gg k_{\mathrm{B}} T\right)$, as $1-k_{\mathrm{B}} T /\left(M_{\mathrm{p}} H\right)$ [202].

The experimental criteria for superparamagnetism are (i) there is no hysteresis in the magnetization curve, and (ii) the magnetization curves at different $T$ must superpose in a plot of $M_{\mathrm{sp}}$ vs $H / T$ [228], which may be influenced by a broad distribution of $D$, and changes in $M_{\mathrm{s}}$ induced by $T$ or anisotropy effect.

\section{ANTIFERROMAGNETIC PROPERTIES}

A simple antiferromagnet can be visualized as consisting of two magnetic sublattices A and B such that the nearest neighbors of the atoms of $\mathrm{A}$ are atoms belonging to $\mathrm{B}$, and vice versa. Any atomic magnetic moments belonging to different sublattices have an antiparallel orientation with an AFM exchange $(J<0)$. As $T$ increases, the thermal agitation energy begins to become comparable to $E_{\text {exc,AFM }}$ and eventually AFM ordering disappears at the Néel temperature $T_{\mathrm{N}}$. For the case that the moments of both sublattices have the same magnitude and they are oriented in opposite directions, one finds that the total magnetization of an antiferromagnet is essentially zero (at least at $T=0$ ) [88].

In comparison with FM metallic elements or alloys, the chemically stable AFM systems such as rutile-type AFM fluorides $\left(\mathrm{FeF}_{2}, \mathrm{CoF}_{2}, \mathrm{MnF}_{2}\right), \mathrm{NiFe}_{2} \mathrm{O}_{4}, \mathrm{CoO}, \mathrm{NiO}, \mathrm{MnO}$, and $\mathrm{CuO}$ are more suitable to investigate the finite-size effect. Thus, in this section, special attention is paid to the phase stability of AFM nanocrystals due to its vital importance for the design of devices and the choice of materials.

\subsection{Size Dependence of the Néel Temperature}

$T_{\mathrm{N}}$ is an important physical quantity for AFM with indicating the phase stability of AFM. As indicated by Néel in his seminal work, as $D$ decreases, a net magnetic moment is produced due to the nonexact compensation of the two magnetic sublattice, i.e., imbalance in the number of "up and down" spins [229]. This results in the inherent size dependence. For free-standing AFM nanocrystals, or AFM nanocrystals embedded in or supported by nonmagnetic matrix, their $T_{\mathrm{N}}(D)$ continuously decreases with dropping $D$ due to also the increase of surface/volume ratio as observed in systems of $\mathrm{FeF}_{2} / \mathrm{ZnF}_{2}[230,231]$ superlattices, and $\mathrm{CoO} / \mathrm{SiO}_{2}$ [232,233], $\mathrm{CoO} / \mathrm{MgO}$ [234], $\mathrm{NiO} / \mathrm{MgO}$ [234,235], $\mathrm{Ho} / \mathrm{Nb} / \mathrm{Y}$, $\mathrm{Ho} / \mathrm{Y} / \mathrm{Nb}$ [128] thin films, as well as $\mathrm{NiO}$ [236], $\mathrm{CoO}$ [237] and $\mathrm{MnO}$ [238] nanoparticles, and $\mathrm{CuO}$ nanoparticles [239242], nanorods [242]. Compared with the superlattices of
AFM layers with nonmagnetic interlayers [243,244], because of the interlayer magnetic coupling [245-248], weaker finite size effect for the superlattices of AFM/AFM insulators with exhibiting single transition temperatures have also been observed in the superlattices systems of $\mathrm{FeF}_{2} / \mathrm{CoF}_{2}$ [249] and $\mathrm{NiO} / \mathrm{CoO}[250]$ measured by thermal expansion and magnetic susceptibility, respectively.

Whereas, for thin AFM films deposited on AFM or FM bulk substrates, such as $\mathrm{CoO} / \mathrm{NiO}$ [234] and $\mathrm{CoO} / \mathrm{Fe}_{3} \mathrm{O}_{4}$ [251], $T_{\mathrm{N}}(D)$ of AFM films increases as $D$ is reduced [234,251] due to the vicinity effect at $\mathrm{NiO} / \mathrm{CoO}$ and $\mathrm{Fe}_{3} \mathrm{O}_{4} / \mathrm{CoO}$ interfaces, where the exchange coupling of $\mathrm{CoO}$ is enhanced $[115,234,251]$. This differs from the experimental results of $\mathrm{Fe} / \mathrm{Cr}(001)$, whose $T_{\mathrm{N}}(D)$ decreases with dropping $D[252,253]$, which could be induced by the spinfrustration effect in the vicinity of the rough $\mathrm{Fe} / \mathrm{Cr}(001)$ interfaces where the interfacial exchange energy can be minimized only locally and frustration of the interfacial spins occurs since $\mathrm{Fe}$ and $\mathrm{Cr}$ have magnetical long-range order $[252,253]$.

Two theoretical models have been proposed for $T_{\mathrm{N}}(D)$ function of AFM nanocrystals. One is the finite-size scaling law employed by Ambrose and Chien [232] for thin $\mathrm{CoO}$ films,

$$
T_{\mathrm{N}}(D) / T_{\mathrm{N}}(\infty)=1-\left(\xi_{0} / D\right)^{\lambda}
$$

with $\xi(T)=\xi_{0}\left[1-T / T_{\mathrm{N}}(\infty)\right]^{-v}$, where $\xi_{0}$ is the extrapolated correlation length at $T=0 \mathrm{~K}$ [120]. The best fit values of $\lambda$ $=1.54 \pm 0.05, \xi_{0}=2.0 \pm 0.1 \mathrm{~nm}$, and $T_{\mathrm{N}}(\infty)=300 \pm 5 \mathrm{~K}$ are obtained [232].

Another is the empirical relation for $\mathrm{Cr}[252,253]$ and Ho thin films [128],

$$
T_{\mathrm{N}}(\infty) / T_{\mathrm{N}}(D)=1+C_{0}{ }^{\prime}\left(D-D_{0}{ }^{\prime}\right)^{-\lambda^{\prime}}
$$

where $C_{0}{ }^{\prime}$ is a constant, $D_{0}{ }^{\prime}$ represents the zero offset in the finite thickness of films, and $\lambda^{\prime}$ is a nonuniversal parameter [121]. For thin Cr films, $D_{0}{ }^{\prime}=4.2 \mathrm{~nm}$ [252] suggests a close relation to the bulk period of the spin-density wave of 6 to $7.8 \mathrm{~nm}$ [254], and the separation of the finite-size effect in these multilayers is impeded by the strong influence of the interfaces. The magnetic behavior of thin Ho films closely resembling that of $\mathrm{Cr}$ [252] can be observed with a particularly interesting difference: $D_{0}{ }^{\prime}=(10.8 \pm 0.5) h \mathrm{~nm}$ for Ho is much closer to the period of the bulk AFM superstructure than for $\mathrm{Cr} / \mathrm{Fe}$ system [128].

Recent theoretical study has also concerned with the magnetic properties of Ho films based on a Heisenberg Hamiltonian within the $J_{1}-J_{2}$ model [87], and the five and six-constant models taking into account $J$ also for $i-j>2$ $[255,256]$. In addition, the interlayer exchange is also considered. The calculated $T_{\mathrm{N}}(D)$ can be consistent with the experimental measurement of Refs. [87] and [128].

Although Eqs. (52), (53) can also fit the experimental data of thin $\mathrm{CoO}, \mathrm{Cr}$ and Ho films [128,232,252,253], it strictly hold only when $D$ is large with the help of the adjustable parameters $\xi_{0}, \lambda$ and $C_{0}{ }^{\prime}, D_{0}{ }^{\prime}, \lambda^{\prime}$, respectively, which are 
case-sensitive without clear physical meaning. Furthermore, above two relationships cannot describe the superheating $T_{\mathrm{N}}(D)$ with dropping $D[234,251]$.

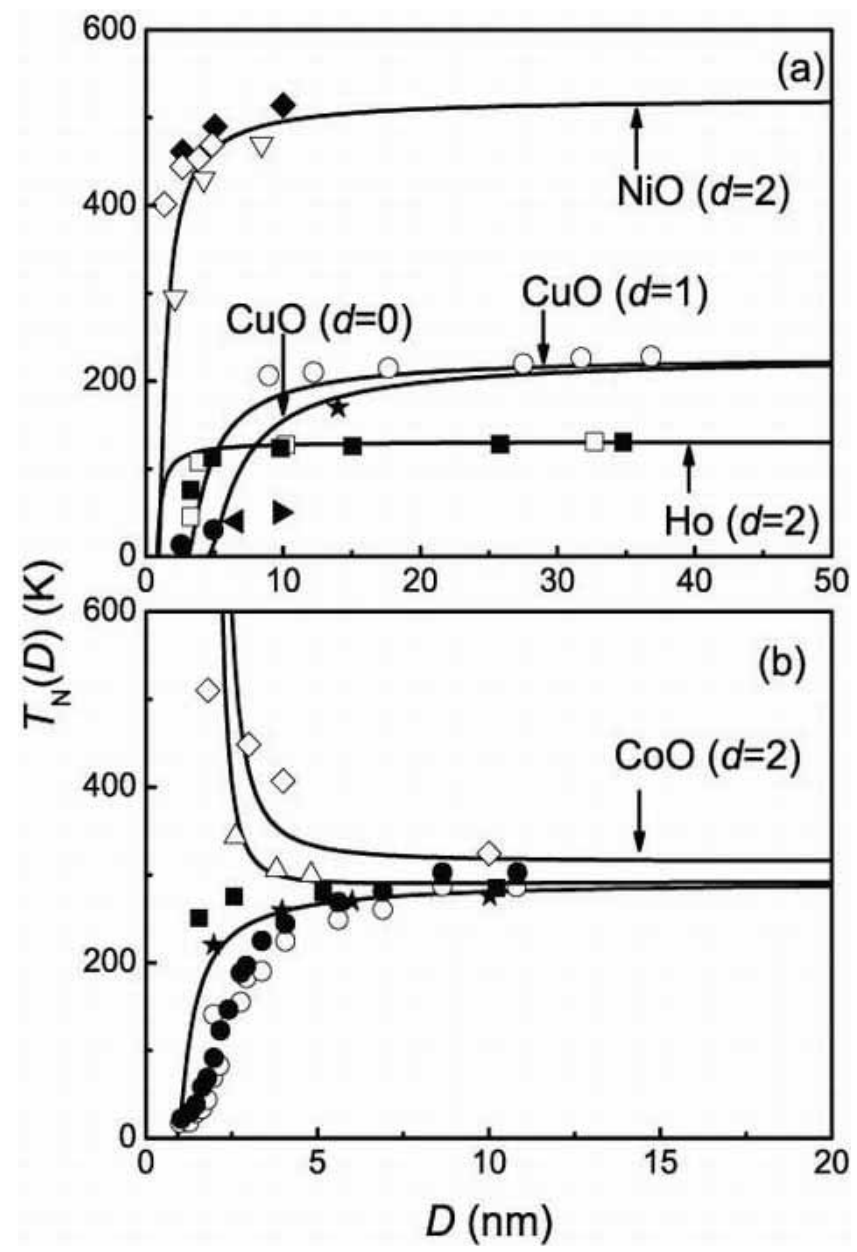

Fig. (11). $T_{\mathrm{N}}(D)$ function comparisons between Eq. (54) and available experimental results: (a) $\mathrm{NiO}$ films $(\diamond, \diamond[234]$ and $\nabla$ [235]), $\mathrm{CuO}$ nanoparticles $(\bigcirc$ [239], $\$ [240], \ [241] and $\$ [242]) and nanorods ( [242]), Ho thin films ( $\square$ and $\square$ [128]) and (b) CoO thin films epitaxially grown on $\mathrm{SiO}_{2}$ or $\mathrm{MgO}$ substrates ( $\boldsymbol{\square}$ and $\star$ [232,233]' and $\bigcirc$ [234]) and on $\mathrm{Fe}_{3} \mathrm{O}_{4}(\triangle$ [251]) and $\mathrm{NiO}(\diamond$ [234]) substrates, respectively. The employed parameters are listed as: (a) $D_{0}=2.738,4.107 \mathrm{~nm}$ for $\mathrm{CuO}$ nanorods and nanoparticles in terms of Eq. (8) with $d=0,1$, and $D_{0}=(2 h) / 2=h=0.7154,0.8420$ $\mathrm{nm}$ for Ho and $\mathrm{NiO}$ thin films with $d=2$, respectively, and $\alpha_{\mathrm{s}}=$ 1.563 for $\mathrm{CuO}$ in terms of Eq. (11) and $\alpha_{\mathrm{i}}=\alpha_{\mathrm{s}}=1.561,1.583$ for Ho and NiO thin films in terms of Eq.(35) with $J_{\mathrm{s}} \approx J_{\mathrm{i}}$ and $J_{\text {sub }} \approx 0$ for epitaxial films on inert substrates; (b) For $\mathrm{CoO}$ deposited on nonmagnetic substrates, the parameters $D_{0}=(2 h) / 2=h=0.8520$ $\mathrm{nm}$ in terms of Eq. (11) with $d=2$, and $\alpha_{\mathrm{i}}=1.544$ according to Eq. (35) with $J_{\mathrm{s}} \approx J_{\mathrm{i}}, J_{\mathrm{sub}} \approx 0$ and $\alpha_{\mathrm{s}}=1.544$ in terms of Eq.(11); for $\mathrm{CoO}$ supported by $\mathrm{Fe}_{3} \mathrm{O}_{4}$ and $\mathrm{NiO}$ substrates, $\alpha_{\mathrm{i}}=0.4139$ and 0.5544 in terms of Eq. (35) with $\alpha_{\mathrm{s}} J_{\mathrm{s}} /\left(J_{\mathrm{s}}+J_{\mathrm{sub}}\right) \approx \alpha_{\mathrm{s}} T_{\mathrm{cm}}(\infty) /\left[T_{\mathrm{cm}}(\infty)+\right.$ $T_{\text {cm,sub }}(\infty)$ ], which is achieved based on the mean-field approximation, $J_{\mathrm{s}} \propto T_{\mathrm{cm}}(\infty)$ and $J_{\text {sub }} \propto T_{\mathrm{cm}, \text { sub }}(\infty)$ or $J_{\mathrm{s}} \propto T_{\mathrm{N}}(\infty)$ and $J_{\text {sub }} \propto$ $T_{\mathrm{N}, \text { sub }}(\infty)$. Other parameters used are listed in Table 4 .

In order to interpret $T_{\mathrm{N}}(D)$ in a unified form, $T_{\mathrm{N}}(D)$ should have a similar form of $T_{\mathrm{c}}(D)$ function based on the molecular field consideration [124]. In terms of Eq. (36), there is,

$$
\begin{aligned}
& T_{\mathrm{N}}(D) / T_{\mathrm{N}}(\infty)=E_{\text {exc,AFM }}(D) / E_{\text {exc,AFM }}(\infty)= \\
& \left\{\exp \left[-\left(\alpha_{\mathrm{s}}-1\right) /\left(D / D_{0}-1\right)\right]+\exp \left[-\left(\alpha_{\mathrm{i}}-1\right) /\left(D / D_{0}-1\right)\right]\right\} / 2
\end{aligned}
$$

Eq. (54) for Ho [128] and $\mathrm{NiO}[232,234,235]$ thin films, $\mathrm{CuO}$ nanoparticles [239-242] and nanorods [242] are shown in Fig. (11a) where their correspondences in full size range are found. Note that $h=2 a$ since the lattice of AFM can be considered to consist of two sublattices with opposite spin direction $[85,88]$.

Table 4. The Parameters Utilized in the Calculations of Eq. (54) for AFM Materials $\left(T_{\mathrm{N}}(\infty)\right.$ is in $\mathrm{K}, S_{\mathrm{m}}(\infty)$ in $\mathrm{J} \cdot \mathrm{g}$ $\operatorname{Atom}^{-1} \cdot \mathrm{K}^{-1}$, and $h$ is in $\left.\mathbf{n m}\right)$

\begin{tabular}{|c|c|c|c|}
\hline & $\boldsymbol{T}_{\mathrm{N}}(\boldsymbol{\infty})$ & $\boldsymbol{S}_{\mathbf{m}}(\boldsymbol{\infty}) \mathbf{b}^{\mathbf{b}}$ & $\boldsymbol{h}$ \\
\hline \hline $\mathrm{Ho}$ & $131.2[128]$ & $6.999[57]$ & $0.7154[57]$ \\
\hline $\mathrm{NiO}$ & $523[234]$ & 7.271 & $0.8420[234]$ \\
\hline $\mathrm{CoO}$ & $315[232] / 293[234]$ & 6.789 & $0.8520[232,234]$ \\
\hline $\mathrm{CuO}$ & $229[242]$ & 7.016 & $0.6845[242]$ \\
\hline
\end{tabular}

${ }^{\mathrm{a}} S_{\mathrm{m}}(\infty)$ of metallic oxides are given as $S_{\mathrm{m}, \mathrm{Mo}}(\infty)=\left[S_{\mathrm{m}, \mathrm{M}}(\infty)+S_{\mathrm{m}, \mathrm{O}}(\infty)\right] / 2$ as a first approximation since experimental data are unavailable, where the subscripts $M$ and $O$ denote the metal and oxygen atoms, respectively.

Similar results for $\mathrm{CoO}$ thin films epitaxially grown on $\mathrm{SiO}_{2}$ or $\mathrm{MgO}$ substrate [232-234] have been shown in Fig (11b). Whereas, for $\mathrm{CoO} / \mathrm{Fe}_{3} \mathrm{O}_{4}$ and $\mathrm{CoO} / \mathrm{NiO}$ systems, there exist strong exchange couplings at the interfaces where the thermal vibration of interface atoms is suppressed and much higher energy is required to disorder the ordering force [235,251]. Therefore, $T_{\mathrm{N}}(D)$ increases as $D$ decreases. The predicted results of Eq. (54) are qualitatively consistent with experimental evidence. Although the free surface of such CoO film has still a tendency to lower $T_{\mathrm{N}}(D)$ value, the total effect of surface+interface leads to the drop of the total energy, and thus the increase of $T_{\mathrm{N}}(D)$ function with decreasing $D$. Furthermore, the stronger the interaction at the interface is, the more $T_{\mathrm{N}}(D)$ function increases [235,251]. Thus, $T_{\mathrm{N}}(D)$ is substrate-dependent. Note also that for the sake of simplicity, exchange bias effect from FM/AFM interface, existence of easy and hard axes, and crystalline anisotropy are neglected.

In terms of lattice vibration instability model, at the Néel transition of AFM, the atomic thermal vibrational energy of nanocrystals is required to disorder $E_{\text {exc,AFM }}$. As pointed out in the experimental observations for free nanocrystals or epitaxial thin films on inert substrates [134,257], there exist different degrees of spin-spin interactions between inner and surface atoms because of the reduction in the number of spin interactions at the surface with increase of lattice vibration $[5,113,12,122,258]$. For thin films with strong film/substrate interaction, the vibration of atoms there is depressed. Thus, the thermal vibration energy to disorder the AFM ordering state of nanocrystals could increase or decrease depending on the interface states [124]. So does $T_{\mathrm{N}}(D)$ function.

As $D>10 D_{0}$, Eq. (54) can be simplified as a first order approximation, $T_{\mathrm{N}}(D) / T_{\mathrm{N}}(\infty)=1-\left(\alpha_{\mathrm{s}}+\alpha_{\mathrm{i}}-2\right) D_{0} /(2 D)$ with $\alpha_{\mathrm{i}}=$ $\left[1+2 S_{\mathrm{vib}}(\infty) /(3 R)\right] J_{\mathrm{s}} / J_{\mathrm{i}}, \alpha_{\mathrm{s}}=1+2 S_{\mathrm{vib}}(\infty) /(3 R)$ and $D_{0}=2(3-d) h$. Thus, $T_{\mathrm{N}}(D)$ function is related to not only the nature of 
AFM materials, but also the magnetic coupling of AFM nanocrystal with matrix, as well as $D$ and $d$.

\subsection{The Morin Transition}

Bulk $\alpha-\mathrm{Fe}_{2} \mathrm{O}_{3}$ (hematite) possesses the corundum-type crystal structure with an AFM spin ordering, which exhibits a first-order magnetic transition at the Morin temperature $T_{\mathrm{M}}{ }^{0}=263 \mathrm{~K}$. The superscript 0 implies that the general Morin temperature $T_{\mathrm{M}}{ }^{\mathrm{Ha}}$, which depends on the applied magnetic field $H_{\mathrm{a}}$, has been extrapolated to zero-field case. Between this transition and the normal $T_{\mathrm{N}}(\infty)=961 \mathrm{~K}$, the spins of $\alpha-\mathrm{Fe}_{2} \mathrm{O}_{3}$ are in (111) basal plane except for a slight spin canting out of the plane [259], which induces a small net weak FM moment. Whilst below $T_{\mathrm{M}}{ }^{0}$ the spins are aligned AFM along [111] axis. The Morin transition arises from a competition between the local ionic anisotropy term from spin-orbit coupling and the long-range dipolar anisotropy term $[260,261]$. The former tends to make spins direct themselves along $\pm z$-axis, while the latter does spins lying in $x y$-plane [261].

For $\alpha-\mathrm{Fe}_{2} \mathrm{O}_{3}$ nanoparticles, $T_{\mathrm{M}}{ }^{0}(D)$ function decreases with $D$ and vanishes at $D \sim 8 \mathrm{~nm}$ [262-268]. Theoretical work showed that the Morin transition depends on $a$ via $a$ dependent dipolar anisotropy term [260], and the size effect of $T_{\mathrm{M}}{ }^{0}(D)$ has been induced by the change of the dipolar magnetic field with a lattice expansion $[263,269,270]$. This is in partial agreement with observed pressure dependencies of $T_{\mathrm{M}}^{0}[271,272]$.

At $T=0 \mathrm{~K}$, a sufficiently large $H_{\mathrm{a}}$ can results in occurrence of the so-called spin-flop reorientation, which can be denoted as $H_{\mathrm{am}}{ }^{0}$ whose size dependence is stronger than $T_{\mathrm{M}}{ }^{0}(D)^{\prime} \mathrm{s}[267,268]$.

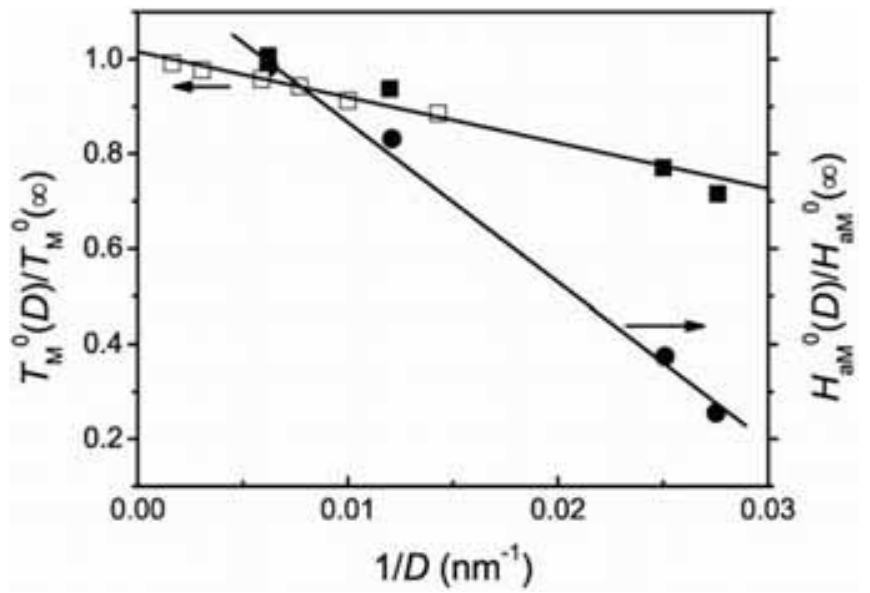

Fig. (12). $T_{\mathrm{M}}{ }^{0}(D) / T_{\mathrm{M}}{ }^{0}(\infty)$ and $H_{\mathrm{aM}}{ }^{0}(D) / H_{\mathrm{aM}}{ }^{0}(\infty)$ functions of $\alpha$ $\mathrm{Fe}_{2} \mathrm{O}_{3}$. The symbols $\square[264] \square[267,268]$, and $\bullet[267,268]$ denote the experimental results of $T_{\mathrm{M}}{ }^{0}(D) / T_{\mathrm{M}}{ }^{0}(\infty)$ and $H_{\mathrm{aM}}{ }^{0}(D) / H_{\mathrm{aM}}{ }^{0}(\infty)$, respectively.

Fig. (12) shows the plots of both $T_{\mathrm{M}}{ }^{0}(D)$ and $H_{\mathrm{aM}}{ }^{0}(D)$ functions. The least squares method gives the following relations [264] of $T_{\mathrm{M}}{ }^{0}(D) / T_{\mathrm{M}}{ }^{0}(\infty)=1-C_{2} / D$ and $H_{\mathrm{aM}}{ }^{0}(D) / H_{\mathrm{aM}}{ }^{0}(\infty)$ $=1-C_{3} / D$ with $C_{2}$ and $C_{3}$ being constants. The linear behavior indicates that the size dependence of the functions should be mainly driven by surface effect [267,268]. In addition, strains [273], crystal defects such as grain boundaries and vacancies [274], and stoichiometry deviations drop also $T_{\mathrm{M}}{ }^{0}(D)[275]$.

\section{EXCHANGE BIAS IN FM/AFM HETEROSTRUC- TURES}

The basic energies of FM or AFM are $E_{\text {exc,FM }}$ or $E_{\text {exc,AFM }}$ and $E_{\text {ani }}$. The former controls magnetic ordering and the latter does the preferred orientation. A ferromagnet generally has a large $E_{\text {exc,FM }}$ but a relatively small $E_{\text {ani, }}$, which makes FM order stable at high $T$ but the orientation may not be, particularly if $D$ is in a few nanometers. In contrast, many antiferromagnets have large $E_{\text {ani }}$ with stable orientations. In the light of both advantages of FM and AFM, FM/AFM heterostructures are useful for modifying and controlling magnetic characteristics because the exchange coupling between them can produce a FM behavior with stable order and high anisotropy [276]. In such a structure, the anisotropy may behave unidirectionally, which results in exchange bias phenomenon. Since its discovery in the system of $\mathrm{Co} / \mathrm{CoO}$ particles in 1956 by Meiklejohn and Bean [277], exchange bias was observed in many different systems containing FM/AFM interface, such as small FM particles covered with their AFM or ferromagnetic native oxide $(\mathrm{Co} / \mathrm{CoO}$ [278280], $\mathrm{Ni} / \mathrm{NiO}$ [281,282], $\mathrm{Fe} / \mathrm{FeO}$ [283], $\mathrm{FeCo} / \mathrm{FeCoO}$ [284], $\mathrm{Fe} / \mathrm{Fe}_{3} \mathrm{O}_{4}[281,285]$ ), FM films on AFM single crystals [286,287] and thin films [288]. Due to the important applications such as permanent magnet materials, high density recording media [289], domain stabilizers in recording heads [290], spin-valve devices and giant magnetoresistance (GMR) type devices [291,292], the exchange bias phenomenon has been extensively investigated in the past decades [276,293-295].

The exchange bias field $H_{\mathrm{e}}$ is commonly manifested as the shift of hysteresis-loop along a magnetic field axis when a ferromagnet in contact with an antiferromagnet [277] or a spin glass [296] is cooled through $T_{\mathrm{N}}$ of AFM or spin glass transition temperature $T_{\mathrm{sg}}$ of spin glass, respectively. It should be noted that $T_{\mathrm{c}}>T_{\mathrm{N}}$ or $T_{\mathrm{sg}}$. In general the shift of $H_{\mathrm{e}}$ is towards negative fields, namely $H_{\mathrm{e}}<0$ if one defines the direction of the cooling field $H_{\mathrm{cf}}$ as positive. However, $\mathrm{FeF}_{2} / \mathrm{Fe}$ bilayers exposed to large cooling fields $\left(H_{\mathrm{cf}} \sim 1 \mathrm{~T}\right)$ can exhibit positive exchange bias, i.e. $H_{\mathrm{e}}>0$ [297].

In the FM/AFM heterostructures, in addition to the appearance of unidirectional anisotropy bringing out $H_{\mathrm{e}} \neq 0$, the most important property is the thermal stability of $H_{\mathrm{e}}$ denoted as the blocking temperature $T_{\mathrm{B}}$ at which the exchange bias vanishes. Usually, $T_{\mathrm{B}}<T_{\mathrm{N}}(\infty)$ (at least slightly) (see the Tables 2-4 in Ref. [293]) and $H_{\mathrm{e}}$ and $T_{\mathrm{B}}$ are sizedependent, which is emphasized in this section. Besides, $H_{\mathrm{e}}$ and $T_{\mathrm{B}}$ are also functions of AFM orientation (compensated versus uncompensated AFM surface [298-303] and in-plane versus out-of-plane AFM spins [298,299,302]), of FM/AFM interface disorder (roughness [299,301,302,304-306], crystallinity [307,308], grain size [309,310], of interface impurity layers [311]), and of strain effect [312-314], of stoichiometry [293] or of presence of multiple phases [315] and so forth. 


\subsection{FM Thickness Dependence of Exchange Bias Field}

For systems consisting of FM thin films with thickness $t_{\mathrm{FM}}$ and AFM substrate $(D \rightarrow \infty)$, the first attempt to develop an intuitive model for exchange bias seems to be Meiklejohn [278], who suggested that the shift of hysteretic loop was due to a large anisotropy in AFM and a weaker exchange energy coupling FM and AFM [277,278]. The energy per unit area of an exchange bias system can be written as [278], $E=-H_{\mathrm{a}} M_{\mathrm{FM}} t_{\mathrm{FM}} \cos \left(\theta_{0}-\beta_{0}\right)+K_{\mathrm{FM}} t_{\mathrm{FM}} \sin ^{2}\left(\beta_{0}\right)+K_{\mathrm{AFM}} D \sin ^{2}\left(\alpha_{0}\right)-$ $J_{\text {INT }} \cos \left(\beta_{0}-\alpha_{0}\right)$, where $J_{\text {INT }}=J_{\mathrm{FM}-\mathrm{AFM}} S_{\mathrm{FM}} S_{\mathrm{AFM}}$ denotes the interface coupling constant of FM/AFM, $\alpha_{0}, \beta_{0}, \theta_{0}$ show the angles between AFM sublattice magnetization and AFM anisotropy axis, the magnetization and the FM anisotropy axis, and the applied field and the FM anisotropy axis. Experimentally, $K_{\mathrm{FM}} t_{\mathrm{FM}} \ll K_{\mathrm{AFM}} D$. Hence, the relationship above can be simplified as $E=-H_{\mathrm{a}} M_{\mathrm{FM}} t_{\mathrm{FM}} \cos \left(\theta_{0^{-}}\right.$ $\left.\beta_{0}\right)+K_{\mathrm{AFM}} D \sin ^{2}\left(\alpha_{0}\right)-J_{\text {INT }} \cos \left(\beta_{0}-\alpha_{0}\right) \quad$ [278,293]. Minimizing this equation with respect to $\alpha_{0}$ and $\beta_{0}$ leads to,

$$
H_{\mathrm{e}}\left(t_{\mathrm{FM}}\right)=J_{\mathrm{INT}} /\left(a^{2} M_{\mathrm{FM}} t_{\mathrm{FM}}\right)
$$

In term of Eq. (55), the order of magnitude of $H_{\mathrm{e}}\left(t_{\mathrm{FM}}\right)$ depends on the unknown parameter $J_{\mathrm{FM}-\mathrm{AFM}}$. If $J_{\mathrm{FM}} \geq J_{\mathrm{FM}-\mathrm{AFM}}$ $\geq J_{\mathrm{AFM}}$, the resulting value for $H_{\mathrm{e}}$ is orders of magnitude larger than that of the experimental observed one [278].

To explain the above discrepancy, two main theoretical approaches have been pursued. On the basis of Néel's contributions [316], Mauri et al. extended the idea of planar domain walls originating at a smooth AFM interface [317] to effectively lower the interfacial energy cost of reversing FM layer without removing the condition of strong interfacial FM/AFM coupling. In this model, they proposed the formation of a planar domain wall in AFM side of the interface. With the magnetization reversal of FM layer, the increase in interfacial exchange energy equals the energy per unit area of an AFM domain wall $2\left(A_{\mathrm{AFM}} K_{\mathrm{AFM}}\right)^{1 / 2}$. Thus, $H_{\mathrm{e}}\left(t_{\mathrm{FM}}\right)$ function can be modified as [317],

$$
H_{\mathrm{e}}\left(t_{\mathrm{FM}}\right)=2\left(A_{\mathrm{AFM}} K_{\mathrm{AFM}}\right)^{1 / 2} /\left(M_{\mathrm{FM}} t_{\mathrm{FM}}\right)
$$

Consequent models [318-320] that have extended Mauri's concept have also shown limited success in fully explaining the exchange bias effect.

Malozemoff [321-323] argued that an ideal interface was unrealistic, and roughness leading to magnetic defects gave rise to local random fields, yielding unidirectional anisotropy. Given the random field and assuming a region with a single domain of FM layer, AFM film will break up into domain-like regions to minimize the net random unidirectional anisotropy. Unlike Mauri et al's model, AFM domain walls are normal to the interface. Once these domains are fixed, flipping FM orientation causes an energy change per unit area of $E_{0}=4 z_{0} J / \pi a L$ with $z_{0}$ being a number of order unity and $L=\pi\left(A_{\mathrm{AFM}} / K_{\mathrm{AFM}}\right)^{1 / 2}$ [321]. Therefore, the modified expression of $H_{\mathrm{e}}\left(t_{\mathrm{FM}}\right)$ can be given by [321-323],

$$
H_{\mathrm{e}}\left(t_{\mathrm{FM}}\right)=2 z_{0}\left(A_{\mathrm{AFM}} / K_{\mathrm{AFM}}\right)^{1 / 2} /\left(\pi^{2} M_{\mathrm{FM}} t_{\mathrm{FM}}\right)
$$

Eqs. (55)-(55) present a common trend of $H_{\mathrm{e}}\left(t_{\mathrm{FM}}\right) \propto 1 / t_{\mathrm{FM}}$ as experimentally observed. However, only Eqs. (56) and (55) could predict correct $H_{\mathrm{e}}$ values under certain conditions.

\subsection{Dependence of $T_{\mathrm{B}}(D)$ in Exchange Biased FM/AFM Bilayers on AFM Thickness}

$T_{\mathrm{B}}$ denotes the thermal stability of exchange biased FM/AFM bialyers, which is of concern for the design of magnetic heads and governs the choice of the biasing materials [293-295]. At $T_{\mathrm{B}}, H_{\mathrm{e}} \rightarrow 0$, which is equal to the shift of the hysteresis loop. As $D$ decreases, $T_{\mathrm{B}}(D)$ decreases in $\mathrm{Fe}_{3} \mathrm{O}_{4} / \mathrm{CoO}[126,251,324], \mathrm{NiO} / \mathrm{NiFe}$ [325], CoNiO/NiFe [325], IrMn/NiFe [325], Py/IrMn [326], NiFe/IrMn [327], $\mathrm{CoFe} / \mathrm{IrMn}$ [328,329], IrMn/NiFe [329], FeMn/NiFe [330], $\mathrm{MnPt} / \mathrm{CoFe}$ [331] and $\mathrm{FeF}_{2} / \mathrm{Fe}$ bilayer systems [332]. Empirical $T_{\mathrm{B}}(D)$ functions have been proposed to fit the corresponding experimental data. One of them, based on the thermal fluctuation model for polycrystalline AFM films, is expressed as [327,333,334],

$$
T_{\mathrm{B}}(D) / T_{\mathrm{B}}(\infty)=1-\left(\xi_{1} / D\right)^{\delta}
$$

where $\xi_{1}=J_{\mathrm{INT}} /\left(2 K_{\mathrm{AFM}} r a\right)$ corresponds to the correlation length, $\delta$ is a constant.

Eq. (56) can also be obtained by extending $T_{\mathrm{N}}(D)$ function $[251,325,331,332,335]$. However, this extension contradicts recent work [251], which suggests that the variation of $T_{\mathrm{B}}(D)$ differs from that of $T_{\mathrm{N}}(D) . T_{\mathrm{N}}(D)$ could increase with decreasing $D$ because of the proximity effect of the adjacent FM layer [251]. In addition, within $T_{\mathrm{B}}(D)$ function of $\mathrm{FeF}_{2} / \mathrm{Fe}$ system, the extracted parameter $\lambda=0.8$ in Eq. (56) is physically unclear, and is much smaller than $\lambda=1.56$ and 1.42 for AFM derived from the Ising and Heisenberg models, respectively [332]. Furthermore, Eq. (56) is valid only for larger $D$ values.

\subsubsection{Lattice Vibration Instability Model for $T_{B}(D)$ Func- tion}

$H_{\mathrm{e}}(\infty)$ at $0 \mathrm{~K}$ or $H_{\mathrm{e} 0}(\infty)$ is related to $E_{0}(\infty)$ where $H_{\mathrm{e} 0}(\infty)=$ $E_{0}(\infty) /\left(M_{\mathrm{FM}} t_{\mathrm{FM}}\right)$ in the light of Eqs. (55)-(55). In this relationship, $M_{\mathrm{FM}}$ is the fixed saturation magnetization of FM layer because the effect of $t_{\mathrm{FM}}$ on $H_{\mathrm{e}}$ is assumed to be constant in this situation. $E_{0}(\infty)=2\left[A_{\mathrm{AFM}}(\infty) K_{\mathrm{AFM}}(\infty)\right]^{1 / 2}$ and $2 z_{0}\left[A_{\mathrm{AFM}}(\infty) K_{\mathrm{AFM}}(\infty)\right]^{1 / 2} / \pi^{2}$ via Eqs. (56) and (55), respectively [293-295,317,321]. As results, $E_{0}(\infty) \propto$ $\left[J_{\mathrm{AFM}}(\infty) S^{2} K_{\mathrm{AFM}}(\infty)\right]^{1 / 2}$. According to this relationship and the mean field approximation of AFM [], $J_{\mathrm{AFM}}(\infty) S^{2} \propto$ $E_{\mathrm{exc}, \mathrm{AFM}}(\infty)$, and thus, $E_{0}(\infty) \propto\left[E_{\mathrm{exc}, \mathrm{AFM}}(\infty) K_{\mathrm{AFM}}(\infty)\right]^{1 / 2}$. It is understandable that the thermal energy $E_{\mathrm{v}}(T)$ is introduced to decrease the FM/AFM interfacial energy with increasing $T$, namely, $E_{\mathrm{T}}(\infty)=E_{0}(\infty)-E_{\mathrm{v}}(T)$, where $E_{\mathrm{v}}(T)=k_{\mathrm{B}} T$ in the light of Einstein's relationship. Taking $E_{T_{B}}(\infty)=0$ as reference, $k_{\mathrm{B}} T_{\mathrm{B}}(\infty) \propto\left[E_{\mathrm{exc}, \mathrm{AFM}}(\infty) K_{\mathrm{AFM}}(\infty)\right]^{1 / 2}$ in terms of the above equation at $T_{\mathrm{B}}(\infty)$. If $\mathrm{AFM}$ nanocrystals remain the corresponding bulk crystalline structure, and the domain walls of AFM layer are perpendicular to the FM/AFM interface $[326,333,334,336], T_{\mathrm{B}}(D)$ can be generalized from the above 
equation, i.e., $k_{\mathrm{B}} T_{\mathrm{B}}(D) \propto\left[E_{\mathrm{exc}, \mathrm{AFM}}(D) K_{\mathrm{AFM}}(D)\right]^{1 / 2}$. Namely [288],

$$
\begin{aligned}
& T_{\mathrm{B}}(D) / T_{\mathrm{B}}(\infty)= \\
& \left\{\left[E_{\mathrm{exc}, \mathrm{AFM}}(D) K_{\mathrm{AFM}}(D)\right] /\left[E_{\mathrm{exc}, \mathrm{AFM}}(\infty) K_{\mathrm{AFM}}(\infty)\right]\right\}^{1 / 2}
\end{aligned}
$$

Based on the Ising model, $K_{\mathrm{AFM}}(\infty) \propto E_{\text {exca }}(\infty)=Q_{\mathrm{e}} E-$ exc,AFM $(\infty) / 2$ is assumed, where $Q_{\mathrm{e}}$ is the exchange anisotropy parameter among the three spin components [87]. If $Q_{\mathrm{e}}$ is a weak function of $D$, extending above relationship to nanometer size leads to $K_{\mathrm{AFM}}(D) \propto E_{\text {exca }}(D)=Q_{\mathrm{e}} E-$ exc,AFM $(D) / 2$ [288]. In this deduction, only $K_{\mathrm{AFM}}(D)>0$ is considered. SRT and the effects of atomic step edges and the strain are neglected for simplicity [87,92]. Thus, $K_{\mathrm{AFM}}(D) / K_{\mathrm{AFM}}(\infty)=E_{\text {exc,AFM }}(D) / E_{\text {exc,AFM }}(\infty)$. Substituting this equation into Eq. (59), there is,

$$
T_{\mathrm{B}}(D) / T_{\mathrm{B}}(\infty)=E_{\text {exc,AFM }}(D) / E_{\text {exc,AFM }}(\infty)
$$

For coherent FM/AFM structures, $E_{0}(\infty)$ determines the magnitude of $T_{\mathrm{B}}(\infty)$ [325-328] while AFM field decides $T_{\mathrm{B}}(D)$ function. Since we consider only the ratio of $T_{\mathrm{B}}(D) / T_{\mathrm{B}}(\infty)$ with experimental $T_{\mathrm{B}}(\infty)$ values, the contribution of $E_{0}(\infty)$ to $T_{\mathrm{B}}(\infty)$ is ignored. Based on this consideration and the mean-field approximation for AFM with sublattice magnetization [85], in terms of Eqs. (54) and (60) [288],

$$
T_{\mathrm{B}}(D) / T_{\mathrm{B}}(\infty)=\exp \left\{-2 S_{\mathrm{vib}}(\infty) /[3 R(D / 2 a-1)]\right\}
$$

\subsubsection{Verification of $T_{B}(D)$ function}

$T_{\mathrm{B}}(D)$ functions of Eq. (57) and known experimental data of $\mathrm{Fe}_{3} \mathrm{O}_{4} / \mathrm{CoO}$ [126,251,324], NiO/NiFe [325], $\mathrm{CoNiO} / \mathrm{NiFe}$ [325], IrMn/NiFe [329], Py/IrMn [326], NiFe/IrMn [327], $\mathrm{CoFe} / \mathrm{IrMn}$ [328,329], IrMn/NiFe [329], FeMn/NiFe [330], $\mathrm{MnPt} / \mathrm{CoFe}$ [331] and $\mathrm{FeF}_{2} / \mathrm{Fe}$ bilayer systems [332] are shown in Fig. (13a-c), where $T_{\mathrm{B}}(D) \rightarrow 0$ as $D$ goes to a few nanometers [293-295]. It should be noted that the $T_{\mathrm{B}}(\infty)$ value of IrMn comes from the corresponding experimental systems of IrMn/NiFe [325,327,329], Py/IrMn [326], CoFe/IrMn [328,329] for better fits with the model predictions while many experimental factors affect $T_{\mathrm{B}}(\infty)$ [293295]. Eq. (57) does not consider these factors since Eq. (57) only predicts the ratio of $T_{\mathrm{B}}(D) / T_{\mathrm{B}}(\infty)$, not $T_{\mathrm{B}}(\infty)$ itself.

According to the definition of $T_{\mathrm{B}}, T_{\mathrm{B}}$ should depend on the strength of FM/AFM interface exchange field [293-295]. This results from the unidirectional exchange anisotropy and the spin-spin exchange interaction of AFM with sublattice magnetization. $T_{\mathrm{B}}(D)$ corresponds to the situation results, at which the AFM spins follow the motion of the FM layer. Thus, $H_{\mathrm{eTB}}(D)=0$ because the unidirectional exchange anisotropy energy is smaller than $E_{0}$ [293-295], where $H_{\mathrm{eTB}}(D)$ denotes the exchange bias at $T_{\mathrm{B}}(D)$.

Eq. (57) also sheds light on $H_{\mathrm{e} 0}(D)$ function in the following form,

$$
\begin{aligned}
& H_{\mathrm{e} 0}(D) / H_{\mathrm{e} 0}(\infty)=T_{\mathrm{B}}(D) / T_{\mathrm{B}}(\infty)= \\
& \exp \left\{-2 S_{\mathrm{vib}}(\infty) /[3 R(D / 2 a-1)]\right\}
\end{aligned}
$$

where the effect of FM layers is set as an invariable. Both $H_{\mathrm{e} 0}(D)$ and $T_{\mathrm{B}}(D)$ functions have the same trend with dropping $D$ as shown in Fig. (14). The results qualitatively corre- spond to available experimental evidences with large scatters $[126,336,337]$. Note that since their $S_{\text {vib }}(\infty) \approx R$ are similar (although the substances are different), the curves based on Eq. (58) are almost the same.

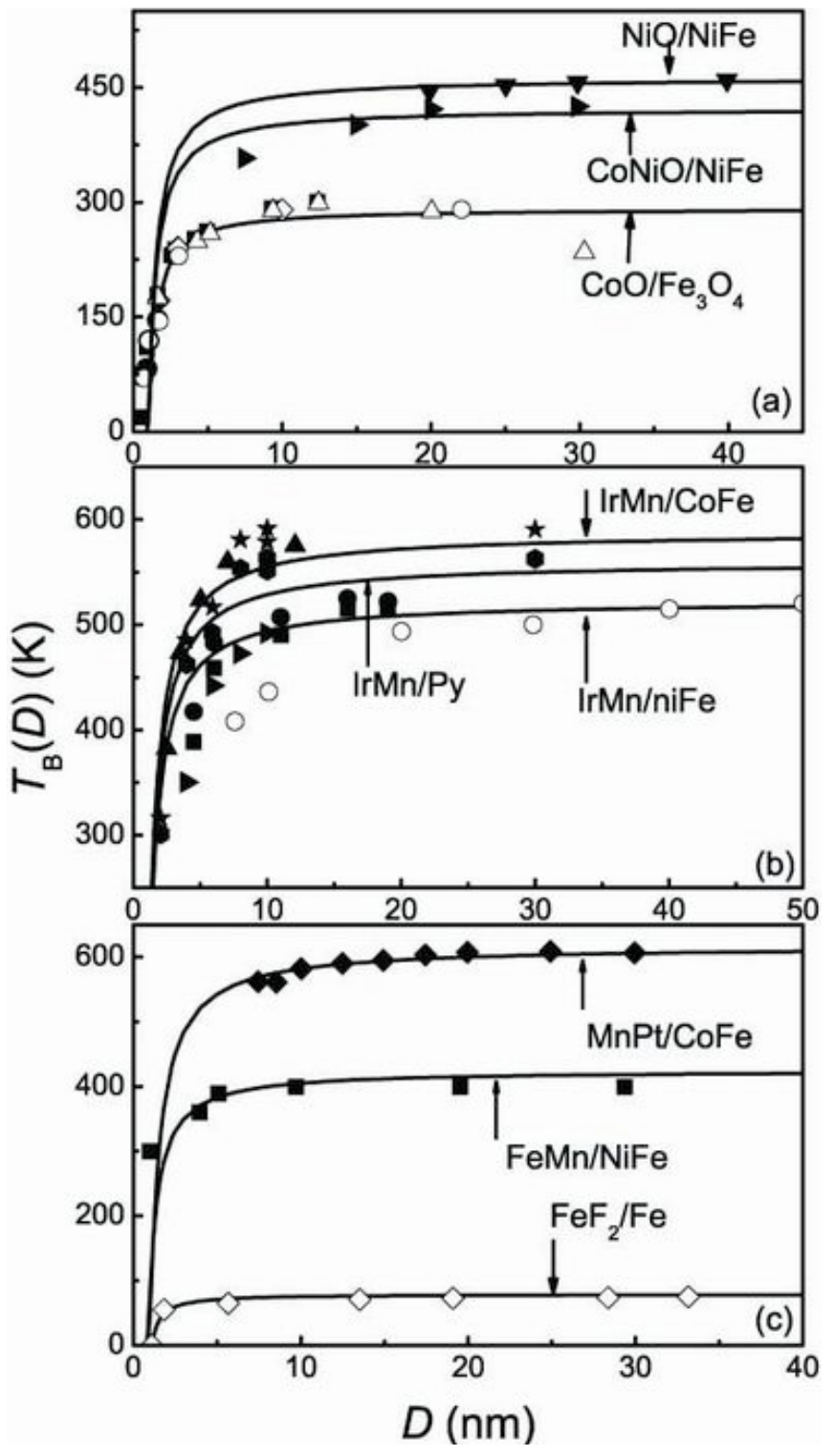

Fig. (13). Comparisons of $T_{\mathrm{B}}(D)$ functions between model predictions in light of Eq. (57) with available experimental evidence: (a) $\mathrm{Fe}_{3} \mathrm{O}_{4} / \mathrm{CoO}(\boldsymbol{\square}$ [251], $\bullet$ [251], $\bullet$ [251], $\boldsymbol{\Delta}$ [251], $\bigcirc$ [126], $\triangle$ [126], $\diamond[324]), \mathrm{NiO} / \mathrm{NiFe}(\boldsymbol{\nabla}$ [325]) and CoNiO/NiFe bilayers (

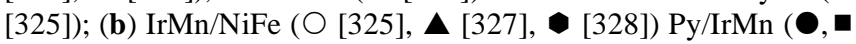
[326]) and CoFe/IrMn bilayers ( $\star$ [328], [329]); (c) $\mathrm{FeMn} / \mathrm{NiFe}$ ( [330]), $\mathrm{MnPt} / \mathrm{CoFe}(\bullet[331])$ and $\mathrm{FeF}_{2} / \mathrm{Fe}$ bilayers systems ( [332]). The necessary parameters are listed in Table $\mathbf{5}$.

Since there exist different degrees of spin-spin interactions between the inner and surface atoms due to $C N$ reduction at AFM surface, $E_{\text {exc,AFM }}(D)$ function and thus $T_{\mathrm{B}}(D)$ and $H_{\mathrm{e} 0}(D)$ functions decrease with dropping $D$ [121,124,288]. As shown in Figs. $(\mathbf{1 3 , 1 4})$, although the related compounds show different spin structures, for example FeMn and MnPt have the non-collinear spin structure and the collinear spin structure, respectively, the model predictions of $T_{\mathrm{B}}(D)$ and 
$H_{\mathrm{e} 0}(D)$ in light of Eqs. (57) and (58) are still in agreement with experimental data.

Table 5. Parameters Utilized in Eq. (57) for AFM Materials $\left(T_{\mathrm{B}}(\infty)\right.$ is in $\mathrm{K}, S_{\text {vib }}(\infty)$ is in $\mathrm{J} \cdot \mathrm{g}$-Atom ${ }^{-1} \cdot \mathrm{K}^{-1}, D_{0}$ and $\xi_{1}$ are in $\mathrm{nm}[\mathbf{2 8 8}]$ )

\begin{tabular}{|c|c|c|c|c|c|}
\hline & $\boldsymbol{T}_{\mathbf{B}}(\infty)$ & $\boldsymbol{S}_{\text {vib }}(\infty)^{\mathbf{a}}$ & $\mathbf{2 a}^{\mathbf{b}}$ & $\boldsymbol{\xi}_{\mathbf{1}}$ & $\boldsymbol{\delta}$ \\
\hline \hline $\mathrm{CoO}$ & 292 & 6.789 & 0.8520 & 1.8 & 1.20 \\
\hline $\mathrm{NiO}$ & 463 & 7.271 & 0.8420 & 1.92 & 1.40 \\
\hline $\mathrm{CoNiO}$ & 423 & 7.899 & 0.8470 & 2.17 & 1.65 \\
\hline $\mathrm{IrMn}$ & $523 / 588 / 560$ & 8.776 & 0.7520 & 3.01 & 1.52 \\
\hline $\mathrm{FeMn}$ & 425 & 7.786 & 0.7140 & 1.00 & 1.60 \\
\hline $\mathrm{MnPt}$ & 616 & 8.764 & 0.7736 & 2.48 & 1.62 \\
\hline $\mathrm{FeF}_{2}$ & 79 & 5.724 & 0.9380 & 0.73 & 0.62 \\
\hline
\end{tabular}

${ }^{a}$ For alloys $\mathrm{AB}, S_{\text {vib }}(\infty)=\left[S_{\mathrm{A} \text { vib }}(\infty)+S_{\mathrm{B} \text { vib }}(\infty)\right] / 2$, where A and B denote different metals, and $S_{\text {vib }}(\infty)=7.493,7.628,9.609,9.584 \mathrm{~J} \cdot \mathrm{g}-$ atom $^{-1} \cdot \mathrm{K}^{-1}$ for $\mathrm{Mn}, \mathrm{Fe}$, Ir, Pt [288], respectively.

${ }^{b} a=0.4260$ [324], 0.4210 [324], 0.3760 [288], 0.3570 [288], 0.3868 [288] and 0.4690 $\mathrm{nm}[288]$ for $\mathrm{CoO}, \mathrm{NiO}, \mathrm{MnIr}, \mathrm{FeMn}, \mathrm{MnPt}$ and $\mathrm{FeF}_{2}$, respectively. $D_{0}=0.4235 \mathrm{~nm}$ for $\mathrm{CoNiO}$ is the mean value of $\mathrm{CoO}$ and $\mathrm{NiO}$.

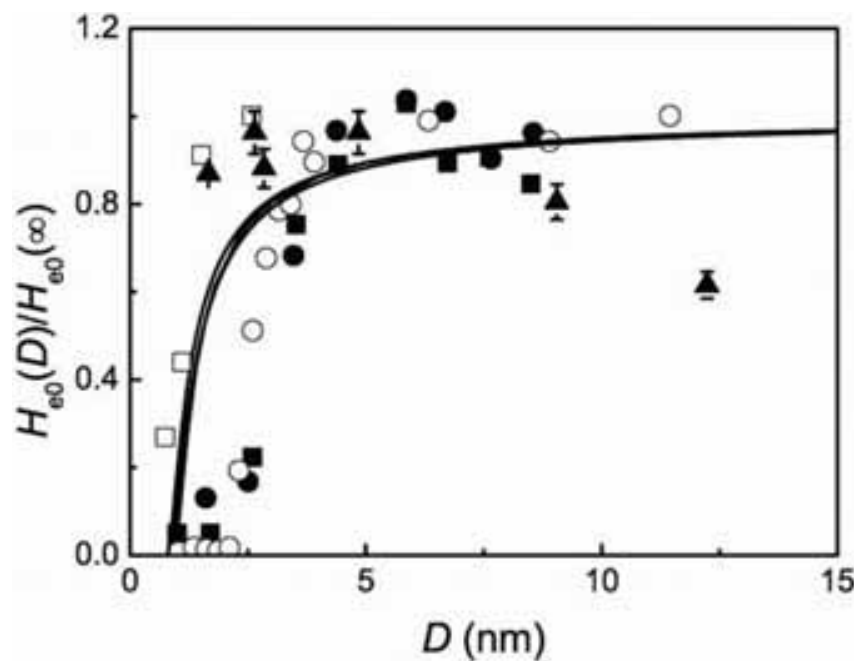

Fig. (14). Comparisons of $H_{\mathrm{e} 0}(D)$ functions between model predictions in terms of Eq. (58) and available experimental results: $\mathrm{CoO} / \mathrm{Fe}_{3} \mathrm{O}_{4}(\square$ and $\boldsymbol{\Delta}[126]), \operatorname{IrMn} / \mathrm{NiFe}(\mathrm{O}[336]), \mathrm{FeMn} / \mathrm{FeNi}$ bilayers ( $\square$ and [337]). The utilized parameters are listed in Table 5. (Reproduced with permission from Ref. [288]. Copyright 2007, IoP)

Using equation of $\exp (-x) \approx 1-x$ when $x$ is small enough, Eq. (57) is simplified as $T_{\mathrm{B}}(D) / T_{\mathrm{B}}(\infty) \approx 1-\left[4 a S_{\text {vib }}(\infty) /(3 R)\right] / D$ or $T_{\mathrm{B}}(D) \propto 1 / D$ at $D>10 a \approx 7-8 \mathrm{~nm}$, which relates $T_{\mathrm{B}}(D)$ closely to the surface/volume ratio of AFM layer and suggests a progressively increasing role of the surface layer with a decreasing $D$. Comparing this equation with Eq. (56), $\delta(D)$ $=\left\{\ln \left[4 a S_{\text {vib }}(\infty) /(3 R)\right]-\ln D\right\} /\left(\ln \xi_{1}-\ln D\right)$. As $D \rightarrow \infty, \delta(\infty)=1$. Thus,

$$
\delta(D) / \delta(\infty)=\left\{\ln \left[4 a S_{\text {vib }}(\infty) /(3 R)\right]-\ln D\right\} /\left(\ln \xi_{1}-\ln D\right)
$$

In Eq. (59), $\xi_{1} \propto J_{\mathrm{INT}} / K_{\mathrm{AFM}}[327,334]$. a similar relationship is $\xi_{0} \propto J_{\mathrm{INT} 0} / K_{\mathrm{AFM} 0}$ can be found in the mean-field theory
[288], with subscript 0 denoting the case of the mean-field approximation. As a result,

$$
\xi_{1} / \xi_{0}=\left(J_{\mathrm{INT}} K_{\mathrm{AFM} 0}\right) /\left(J_{\mathrm{INT} 0} K_{\mathrm{AFM}}\right)
$$

As $\xi_{1}=4 a S_{\text {vib }}(\infty) /(3 R), \delta(D)=1$ in terms of Eq. (59), which corresponds to $\delta$ value of the mean-field theory and leads to $\xi_{0}=4 a S_{\text {vib }}(\infty) /(3 R)$ in light of Eq. (56) $[288,326]$. With a good approximation, $S_{\mathrm{vib}}(\infty) \approx R$ for compounds and metallic atoms, and $a=\sqrt{2} h$ for fcc structure, $\xi_{0} \approx 4 \sqrt{2} h / 3$ being in agreement of the expected $\xi_{0}$ value of $h \sim 2 h$ [115,338]. Since $a$ is a function of $h$, such as $h=\sqrt{2} a / 2$ for $\mathrm{NaCl}$ structure and $h=\left(2 a^{2}+c^{2}\right)^{1 / 2} / 2$ for body-centered tetragonal crystal structure, respectively, $\xi_{0}$ is a function of lattice structures.

Obviously, the experimentally overestimated $J_{\mathrm{INT}}$ value in comparison with $J_{\text {INT0 }}$ results in $\xi_{1}>\xi_{0}$ according to Eq. (60) while $J_{\text {INT }}$ is strongly affected by experimental conditions, as listed in Table 5. In light of the $\xi_{1}$ values of $\mathrm{CoO}$, $\mathrm{NiO}, \mathrm{CoNiO}, \mathrm{IrMn}, \mathrm{FeMn}$ and $\mathrm{MnPt}, \delta(D)$ function determined by Eq. (59) generally increases with decreasing $D$, which is shown in Fig. (15).

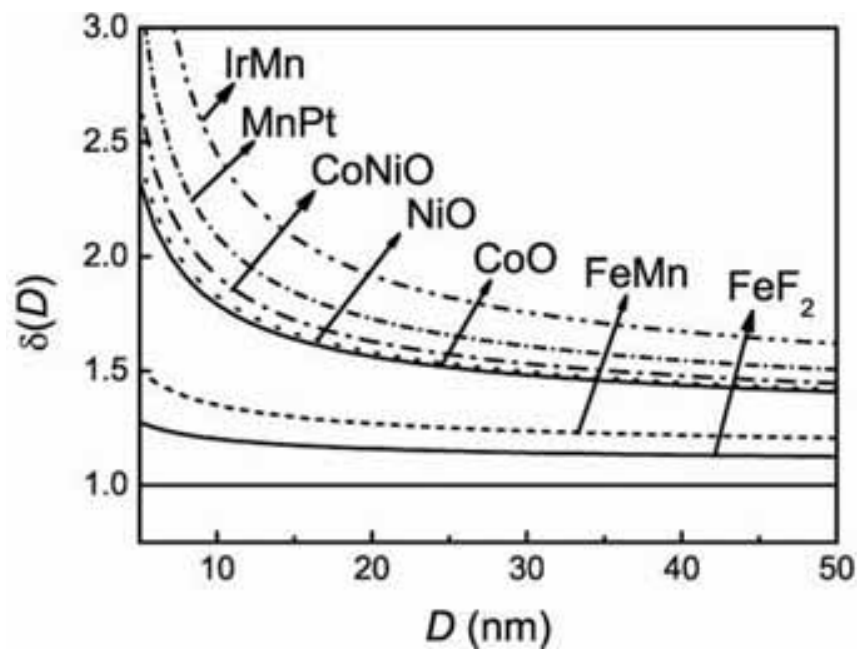

Fig. (15). Shift exponent $\delta(D)$ function in term of Eq. (59) for $\mathrm{CoO}$, $\mathrm{NiO}, \mathrm{CoNiO}$, IrMn, FeMn, MnPt and $\mathrm{FeF}_{2} \mathrm{FM} / \mathrm{AFM}$ bilayers. The used parameters in Eq. (59) are listed in Table 5. (Reproduced with permission from Ref. [288]. Copyright 2007, IoP)

If $J_{\text {INT }}$ value is derived from the Heisenberg model, where the spin-spin exchange coupling in thin films is assumed to be uniform [85,339], $J_{\text {INT }}>J_{\text {INT0 }}, \xi>\xi_{0}$ and $\delta>1$ [327] are also obtained since the exchange coupling near surfaces of thin films in mean-field approximation is expected to be weaker than that in bulk $[113,121,122,339]$. Nevertheless, for AFM materials with much higher anisotropy, such as $\mathrm{FeF}_{2}$ [332], $\xi_{1}$ is predominantly determined by $K_{\mathrm{AFM}}$, and the effect of $J_{\mathrm{INT}}$ is negligible. Thus, $\xi_{1} \approx \xi_{0}$ in terms of Eq. (60) with $J_{\mathrm{INT}} / K_{\mathrm{AFM}} \approx J_{\mathrm{INT} 0} / K_{\mathrm{AFM} 0}$, and $\delta(D) \approx 1$ with a much weak apparent size dependence according as Eq. (59).

As $D>D_{0}$, if $\xi \approx \xi_{0} \approx 4 a S_{\text {vib }}(\infty) /(3 R)$ and $\delta \approx 1$ in Eq. (56), Eq. (56) $\approx$ Eq. (57), in which the parameters are definitely physical on describing all $T_{\mathrm{B}}(D)$ functions in the ex- 
change biased FM/AFM bilayers. As $D \rightarrow h$, Eq. (57) is more suitable for predicting $T_{\mathrm{B}}(D)$ function where energetic changes of internal atoms in AFM layers also contribute to $T_{\mathrm{B}}$.

\section{CONCLUDING REMARKS}

The properties of magnetic materials are obviously dependent not only on intrinsic materials parameters but also on the special types of microstructures. This review presents size dependence of FM, AFM nanocrystals and FM/AFM bilayers on the studies of thermal and phase stabilities, the mechanical properties, and the magnetic properties. The tunable properties will be advantageous in choosing materials for designing and functioning of the devices.

In this review, the lattice thermal instability model is proposed to illustrate the size-induced mechanism behind the unusual behaviors of magnetic nanocrystals. On the basis of thermodynamics, simple and analytical models, without any adjustable parameter, are presented to quantitatively reproduce the relative experimental results of magnetic properties of nanocrystals. Their unified forms not only reveal the physical nature of size and interface dependences, but also interpret the underlying relationships among the thermal and phase stabilities, as well as the magnetic properties. As indicated in this model, the size dependences of magnetic nanocrystals are related to both surface/volume ratio and interface effect. The latter plays significant role in determining the trend of changes of magnetically physical quantities with respect to $D$. For example, due to the strong FM/AFM interface coupling, $T_{\mathrm{c}}(D)$ and $T_{\mathrm{N}}(D)$ of FM and AFM nanocrystals can increase as $D$ is reduced. This implies that there is a feasible method to stabilize these nanocrystals for practical applications.

Our models have predicted the relative difference of nanocrystals and bulk counterparts, while the most properties of the materials have been embodied in the bulk properties. Thus, our models could be realized in a simple and unified form. The success of the above classic thermodynamics in the full size range of materials further enriches the classical thermodynamic theory, and offers a powerful, irreplaceable and unfailing theoretical guidance for the development of materials science. However, since thermodynamics can only describe the statistical behavior of large numbers of molecules, this thermodynamic top-down method cannot be utilized to depict the action of clusters with only a few molecules or atoms. Once $D$ decreases to $D_{0}$, quantum effect is introduced, which results in the disability of this method. In addition, if structures of nanocrystals differ from that of the corresponding bulk, where the electronic distribution varies, this model is invalid since the nanocrystals in the above deduction are assumed to remain in the same crystalline structure as the bulk.

Since the current model mainly considers the sizedependent properties of magnetic nanocrystals of elements and compounds, the further development of model requires extensively extensions to multicomponent systems. Moreover, the size dependence concerned is essentially affected by surface and interface while the effects of grain boundaries

and intergranular phase boundaries are not specially developed, which needs progress as secondary effects.

\section{ACKNOWLEDGEMENTS}

The financial supports by National Key Basic Research and Development Program (Grant No. 2004CB619301) and by "985 Project" of Jilin University are acknowledged.

\section{ABBREVIATIONS AND SYMBOLS USED}

$$
\begin{aligned}
& a, a_{\text {sub }} \quad=\text { Lattice constant where subscript sub show } \\
& \text { magnetic films and substrates } \\
& \text { A }=\text { Exchange stiffness } A=2 J S_{\mathrm{i}} S_{\mathrm{j}} / a \\
& \mathrm{AFM} \quad=\text { Antiferromagnetic } \\
& \text { BOLS = Bond order-length-strength } \\
& B_{\mathrm{s}}(y) \quad=\text { Brillouin function } \\
& c_{1} \quad=\text { An additional condition for different surface } \\
& \text { states } \\
& C=\text { A constant related to } \gamma \\
& C_{0}{ }^{\prime} \quad=\text { A constant } \\
& D \quad=\text { Dimension } \\
& d_{0} \quad=d_{0}=24\left[K(\infty) k_{\mathrm{B}} T_{\mathrm{c}}(\infty) / a\right]^{1 / 2} / M_{\mathrm{s}}^{2}(\infty) \\
& d^{\prime} \quad=d^{\prime}=(2+d) / 3 \\
& D \quad=\text { Thickness of thin films, diameter of } \\
& \text { nanoparticles or nanorods } \\
& D_{1}, D_{2} \quad=\text { Principal diameter of curvature of the crystal } \\
& \text { being in equilibrium on the interface } \\
& =\text { The critical size at which all the atoms or } \\
& D^{\prime} \quad=\text { Finite thickness of films at } T_{\mathrm{c}}(D) \text { or } T_{\mathrm{N}}(D)=0 \\
& \mathrm{~K} \\
& =\frac{4\left(1-v_{a}\right) \bar{S}_{v i b} \bar{H}_{m} h}{\sin \theta \bar{V}_{m} R E\left(a_{m}-a_{s u b}\right)^{2}} \\
& D_{\mathrm{s}} \quad=\text { Single-domain size } \\
& E_{0} \quad=\text { FM/AFM interfacial energy } \\
& E_{\mathrm{a}} \quad=\text { Anisotropy energy barrier } E_{\mathrm{a}}=K V \\
& E_{\text {ani }} \quad=\text { Anisotropy energy } \\
& E_{\mathrm{c}} \quad=\text { Cohesive energy, } E_{\mathrm{c}}=z N_{\mathrm{a}} \varepsilon / 2 \\
& E_{\mathrm{exc}} \quad=\text { Exchange interaction energy } \\
& \mathrm{E}_{\mathrm{b}} \quad=\mathrm{E}_{\mathrm{b}}=\mathrm{E}_{\mathrm{a}}+\mathrm{E}_{\text {int }} \\
& E_{\text {int }} \quad=\text { Interaction energy barrier } \\
& E_{\mathrm{m}} \quad=\text { Elastic modulus } \\
& E_{\mathrm{s}} \quad=\text { Magnetostatic energy } \\
& E_{\mathrm{v}}(T) \quad=\text { Thermal energy } \\
& E_{\mathrm{z}} \quad=\text { Zeeman energy } \\
& \text { EIT = Exchange interface thickness }
\end{aligned}
$$




\begin{tabular}{|c|c|c|c|}
\hline$F$ & $\begin{aligned}= & \text { Fraction of the nearest-neighbor spacing at } \\
& \text { which melting occurs }\end{aligned}$ & $\begin{array}{l}M_{\mathrm{p}} \\
M_{\mathrm{s}}\end{array}$ & $\begin{array}{l}=M_{\mathrm{p}}=M_{\mathrm{s}} V \\
=\text { Saturation magnetization }\end{array}$ \\
\hline$F(D)$ & $\begin{aligned}= & \text { Size dependent function during the deduction } \\
& \text { of } T_{\mathrm{m}}(D) \text { function }\end{aligned}$ & $M_{\mathrm{sp}}(T)$ & $\begin{aligned}= & \text { Temperature-dependent magnetization } \\
& \text { behavior of particles }\end{aligned}$ \\
\hline$F_{\mathrm{m}}$ & $=$ Molecular field & $M(T)$ & $=M(T)=N g S \mu_{\mathrm{B}} B_{\mathrm{s}}(y)$ \\
\hline FM & $=$ Ferromagnetic & $n\left(n_{\mathrm{t}}, n_{\mathrm{s}}, n_{\mathrm{v}}\right)$ & $=$ Atom number of nanocrystals where \\
\hline$G$ & $=$ Landé spectroscopic $g$-factor & & subscripts $\mathrm{t}, \mathrm{s}$ and $\mathrm{v}$ denote total, surafce and \\
\hline$h$ & $=$ Atomic diameter & & interior \\
\hline$H$ & $=$ Hardness & $n_{\mathrm{i}}$ & $=(3-d) h q_{\mathrm{s}} / D$ \\
\hline$H_{\mathrm{a}}$ & $=$ Applied field & $N$ & $=$ Number of molecules per unit volume \\
\hline$H_{\mathrm{c}}$ & $=$ Coercive force & $N_{0}$ & $\begin{aligned}= & \text { Demagnetizing factor of the prolate spheroid } \\
& \text { in the direction of the major axis }\end{aligned}$ \\
\hline$H_{\mathrm{cf}}$ & $=$ Cooling field & $N_{\mathrm{a}}$ & $=$ The Avogadro number \\
\hline$H_{\mathrm{cm}}$ & $=H_{\mathrm{cm}}=2 K(\infty) / M_{\mathrm{s}}(\infty)$ & $N_{\text {eff }}(D)$ & $=N_{\mathrm{eff}}(D) \propto \tan ^{-1}\left[D /\left(2 D_{\mathrm{s}}^{2}+D^{2}\right)^{1 / 2}\right]$ \\
\hline$H_{\mathrm{cs}}$ & $=H_{\mathrm{cs}}=\left(N_{\mathrm{t}}-N_{0}\right) M_{\mathrm{s}}(\infty)$ & $N_{\mathrm{t}}$ & $=$ Demagnetizing factor in any direction at righ \\
\hline$H_{\mathrm{cst}}$ & $=H_{\mathrm{cst}}=3 \lambda_{\mathrm{m}} \sigma_{\mathrm{m}} / M_{\mathrm{s}}(\infty)$ & & angles to the long axis \\
\hline$H_{\mathrm{e}}$ & $=$ Exchange bias & $q_{\mathrm{s}}$ & $=q_{\mathrm{s}}=h_{\mathrm{s}} / h=2 /\left\{1+\exp \left[\left(12-z_{\mathrm{s}}\right) /\left(8 z_{\mathrm{s}}\right)\right]\right\}$ \\
\hline$H_{\mathrm{m}}$ & $=$ Latent heat of fusion & $Q$ & $=$ General physical quantities \\
\hline$H_{\mathrm{M}}{ }^{0}$ & $=$ Spin-flop reorientation field at $T=0 \mathrm{~K}$ & $Q_{\mathrm{a}}$ & $=$ Activation energy for interface migration \\
\hline$J\left(J_{\mathrm{i}}, J_{\mathrm{s}}, J_{\mathrm{sub}}\right)$ & $\begin{aligned}= & \text { Exchange constant or exchange coefficient } \\
& \text { where subscripts } \mathrm{s} \text { and sub show surface and }\end{aligned}$ & $Q_{\mathrm{e}}$ & $\begin{array}{l}=\text { Exchange anisotropy parameter among the } \\
\text { three spin components }\end{array}$ \\
\hline & substrate, respectively and $J_{\mathrm{i}}=J_{\mathrm{s}}+J_{\mathrm{sub}}$ & $Q_{\mathrm{s}}\left(Q_{\mathrm{v}}\right)$ & $=$ General physical quantities located at surface \\
\hline$k_{\mathrm{B}}$ & $=$ The Boltzmann constant & & (within crystals) \\
\hline$k_{\mathrm{d}}$ & $=\mathrm{A}$ positive material constant & $r$ & $=$ Radius of the incompletely filled $\mathrm{d}$ shell \\
\hline$k_{\mathrm{t}}^{\prime}$ & $=\mathrm{A}$ constant in $\chi_{0}^{\prime}=k_{\mathrm{t}}^{\prime} \exp [Q(D) /(2 R T a)]$ & $R$ & $=$ Ideal gas constant \\
\hline$K$ & $=$ Anisotropy constant & $S$ & $=S$-spin \\
\hline$K_{2}$ & $=$ Strength of local uniaxial anisotropy & $S_{\mathrm{b}}$ & $=$ Bulk evaporation entropy of crystals \\
\hline$K_{\mathrm{i}}$ & $=K_{\mathrm{i}}=-3 \lambda_{\mathrm{m}} E_{\mathrm{m}} \varepsilon_{\mathrm{m}} / 2$ & $S_{\mathrm{el}}(\infty)$ & $=$ Electric entropy of melting \\
\hline$K_{\mathrm{s}}\left(K_{\mathrm{v}}\right)$ & $=$ Surface (volume) contribution of $K$ & $S_{\text {pos }}(\infty)$ & $=$ Positional entropy of melting, $S_{\text {pos }}(\infty)=-$ \\
\hline$K_{\text {step }}$ & $=K_{\text {step }}=K_{\text {step,edge }}+K_{\text {step,corner }}$ & & $R\left(x_{\mathrm{A}} \ln x_{\mathrm{A}}+x_{\mathrm{v}} \ln x_{\mathrm{v}}\right)$ \\
\hline$K_{\text {step,edge }}$ & $=$ Magnetic anisotropy induced by step edges or & $S_{\mathrm{m}}(\infty)$ & $=$ Melting entropy \\
\hline$K_{\text {step,corner }}$ & step corners & $S_{\text {vib }}$ & $=$ Vibrational entropy of melting \\
\hline$L$ & $=L=\pi\left(A_{\mathrm{AFM}} / K_{\mathrm{AFM}}\right)^{1 / 2}$ & $\mathrm{t}$ & $=\mathrm{t}=1-\mathrm{T} / \mathrm{T}_{\mathrm{c}}$ \\
\hline$\Delta L$ & $=$ Thickness of surface layer of nanoparticles & $t_{0}$ & $=\mathrm{A}$ constant thickness \\
\hline$L_{\text {exc }}$ & $=$ Exchange length, $L_{\mathrm{exc}}=\left[2 A /\left(\mu_{0} M_{\mathrm{s}}^{2}\right)\right]^{1 / 2}$ & $t_{\mathrm{FM}}$ & $=$ Thickness of FM layer \\
\hline$L_{\mathrm{w}}$ & $=$ Domain wall width, $L_{w}=(A / K)^{1 / 2}$ & $t_{\mathrm{w}}$ & $=$ Terrace width of surface steps \\
\hline$m_{\mathrm{a}}$ & $=$ Atomic mass & $T$ & $=$ Absolute temperature \\
\hline$m_{\perp}, m_{\|}$ & $=$Perpendicular and in-plane magnetic & $T_{\mathrm{a}}$ & $=$ Experimental or ambient temperature \\
\hline & directions & $T_{\mathrm{b}}$ & $=$ Blocking temperature of superparamagnetism \\
\hline M & $=$ Matrix & $T_{\mathrm{B}}$ & $=$ Blocking temperature of exchange biased \\
\hline$M$ & $=$ Molecular weight & & AFM/FM bilayers \\
\hline$M_{0}$ & $=$ Magnitude of the local magnetization vector & $T_{\mathrm{c}}$ & $=$ The Curie temperature \\
\hline$M_{\mathrm{FM}}$ & $=$ Saturation magnetization of FM layer & $T_{\mathrm{m}}$ & $=$ Melting temperature \\
\hline$M_{\mathrm{r}}$ & $=$ Remanent magnetization & $T_{\mathrm{M}}^{0}$ & $=$ The Morin temperature at $H=0$ oe \\
\hline
\end{tabular}




$$
\begin{aligned}
& T_{\mathrm{N}} \quad=\text { The Néel temperature } \\
& \theta=\text { Contact angle between a particle and the } \\
& \text { matrix } \\
& T_{\mathrm{r}} \quad=\text { Spin reorientation transition }(\mathrm{SRT}) \\
& \text { temperature } \\
& T_{\mathrm{sg}}=\text { Spin glass transition temperature } \\
& x_{\mathrm{A}} \quad=x_{\mathrm{A}}=1 /\left(1+\Delta V_{\mathrm{s}} / V_{\mathrm{s}}\right) \\
& x_{\mathrm{v}} \quad=x_{\mathrm{v}}=1-x_{\mathrm{A}} \\
& y \quad=y=2 g S \mu_{\mathrm{B}} \mu_{0} F_{\mathrm{m}} \\
& \Delta U=\text { Energy density difference between the } \\
& \text { nanocrystal and the nanoliquid } \\
& U_{\mathrm{e}} \quad=\text { Elastic energy of magnetic films } \\
& U_{\mathrm{i}} \quad=\text { Film/substrate interface energy } \\
& V \quad=\text { Volume of particles } \\
& V_{\mathrm{m}} \quad=\text { Molar volume } \\
& w \quad=\text { An indicator for the bond nature in BOLS } \\
& \text { model } \\
& z\left(z_{\mathrm{v}}, z_{\mathrm{s}} z_{\mathrm{sv}}\right)=\text { Coordination number }(C N) \text { where subscripts } \mathrm{v} \\
& \text { and s show bulk and surface } C N \text {, and } \mathrm{z}_{\mathrm{sv}}=\mathrm{z}_{\mathrm{S}} / \mathrm{z}_{\mathrm{v}} \\
& z_{0} \quad=\text { A number of order unity }
\end{aligned}
$$
$\theta_{0} \quad=$ Angle between applied field and FM anisotropy axis$$
\Theta_{\mathrm{D}} \quad=\text { The Debye temperature }
$$$$
\alpha_{0}=\text { Angle between AFM sublattice magnetization }
$$$$
\text { and AFM anisotropy axis }
$$$$
\alpha_{\mathrm{s}} \quad=\alpha_{\mathrm{s}}=\sigma_{\mathrm{s}}{ }^{2}(D) / \sigma_{\mathrm{v}}{ }^{2}(D)
$$$$
\alpha_{\mathrm{i}} \quad=\alpha_{\mathrm{i}}=\sigma_{\mathrm{i}}{ }^{2}(D) / \sigma_{\mathrm{v}}{ }^{2}(D)=\alpha_{\mathrm{s}} \sigma_{\mathrm{i}}{ }^{2}(D) / \sigma_{\mathrm{s}}{ }^{2}(D)
$$$$
\beta \quad=\text { Layer thickness of surface liquid }
$$$$
\beta_{0} \quad=\text { Angle between magnetization and FM }
$$$$
\text { anisotropy axis }
$$$$
\chi \quad=\text { Yield strength }
$$$$
\chi_{0} \quad=\text { Bulk yield strength }
$$$$
\chi_{0}^{\prime} \quad=\chi_{0}^{\prime}=k_{\mathrm{t}}^{\prime} \exp \left[Q(D) /\left(2 R T_{\mathrm{a}}\right)\right]
$$$$
\chi_{0}{ }^{\prime \prime} \quad=\text { Temperature-independent term of } \chi_{0}
$$$$
\delta \quad=\text { A critical exponent }
$$$$
\varepsilon \quad=\text { Bond energy }
$$$$
\varepsilon_{\mathrm{m}} \quad=\text { Strain }
$$

$\gamma\left(\gamma_{\mathrm{sl}}, \gamma_{\mathrm{sv}}, \gamma_{\mathrm{lv}}\right)=$ Interface energy where subscripts sl, sv and lv denotes solid-liquid, Solid-vapor and liquidvapor interface

\section{REFERENCES}

[1] Bland J.A.C.; Heinrich B. Ultrathin Magnetic Structures, Vol. I + II, Springer, Berlin, 1994.

[2] Bruno P. Interlayer Exchange Interactions in Magnetic Multilayers, in: Miller J.S.; Drillon M. (Ed.), Magnetism: Molecules to Materials III., Wiley-VCH, Weinheim, 2002.

Aktaş B.; Tagirov L.; Mikailov F. Magnetic Nanostructures, Springer, Berlin, 2006.

[4] Bader S.D. Rev. Mod. Phys., 2006, 78, 1.

[5] Sun C.Q. Prog. Solid State Chem., 2007, 35, 1.

[6] Gleiter H. Prog. Mater. Sci., 1989, 33, 223.

[7] Löffler J.F.; Braun H.B.; Wagner W. Phys. Rev. Lett., 2000, 85, 1990.

[8] Takagi M. J. Phys. Soc. Jpn, 1954, 9, 359.

[9] Zhao M.; Zhou X.H.; Jiang Q. J. Mater. Res., 2001, 16, 3304

[10] Jackson C.L.; McKenna G.B. J. Chem. Phys., 1990, 93, 9002.

[11] Jiang Q.; Shi H.X.; Zhao M. J. Chem. Phys., 1999, 111, 2176.

[12] Zhang Z.; Zhao M.; Jiang Q. Semicond. Sci. Technol., 2001, 16, L33.

[13] Kara A.; Rahman T.S. Phys. Rev. Lett., 1998, 81, 1453.

[14] Hoyt J.J. Phys. Rev. Lett., 2006, 96, 045702.

[15] Jiang Q.; Zhang Z.; Li J.C. Acta Mater., 2000, 48, 4791.

[16] O'Connor C.J.; Tang J.; Zhang J.H. Nanostructured magnetic materials, in: Miller J.S.; Drillon M. (Ed.), Magnetism: Molecules to Materials III., Wiley-VCH, Weinheim, 2002.

[17] Zhou M.Y.; Sheng P. Phys. Rev. B, 1991, 43, 3460.

[18] Ding F.; Rosén A.; Bolton K. Phys. Rev. B, 2004, 70, 075416.

[19] Wen Y.H.; Zhu Z.Z.; Zhu R.Z.; Shao G.F. Physica E, 2004, 25, 47.

[20] Ding F.; Bolton K.; Rosén A. J. Vac. Sci. Technol. A, 2004, 22 1471.

[21] Qi Y.; Cagin T.; Johnson W.L.; Goddard W.A. J. Chem. Phys., 2001, $115,385$.

[22] Herr U.; Jing J.; Birringer R.; Gonser U.; Gleiter H. Appl. Phys. Lett., 1987, 50, 472.

$\eta \quad=\eta=\left(h-h_{\text {sub }}\right) / h$

[23] Childress J.R.; Chien C.L.; Zhou M.Y.; Sheng P. Phys. Rev. B, 1991, 44, 11689

[24] Hou M.; Azzaoui M.E.; Pattyn H.; Verheyden J.; Koops G.; Zhang G. Phys. Rev. B, 2000, 62, 5117 .

[25] Tong W.P.; Tao N.P.; Wang Z.B.; Lu J.; Lu K. Science, 2003, 299 , 686.

[26] Jiang Q.; Zhang S.H.; Li J.C. Solid State Commun., 2004, 130, 581.

[27] Pan C.Y.; Zhang Z.J.; Su X.; Zhao Y.; Liu J.G. Phys. Rev. B, 2004, $70,233404$. 
[28] Shiratsuchi Y.; Yamamoto M.; Bader S.D. Prog. Surf. Sci., (2007) DOI: 10.1016/j.progsurf.2006.08.001.

[29] Sun S.H.; Murray C.B. J. Appl. Phys., 1999, 85, 4325.

[30] Cheon J.; Park J.; Choi J.S.; Jun Y.W.; Kim S.H.; Kim M.G.; Kim Y.M.; Kim Y.J., Proc. Natl. Acad. Sci. USA, 2006, 103, 3023.

[31] Tian C.S.; Qian D.; Wu D.; He R.H.; Wu Y.Z.; Tang W.X.; Yin L.F.; Shi Y.S.; Dong G.S.; Jin X.F.; Jiang X.M.; Liu F.Q.; Qian H.J.; Sun K.; Wang L.M.; Rossi G.; Qiu Z.Q.; Shi J. Phys. Rev. Lett., 2005, 94, 137210.

[32] Mei Q.S.; Lu K. Prog. Mater. Sci., 2007, 52, 1175.

[33] Buffat P.; Borel J.P. Phys. Rev. A, 1976, 13, 2287.

[34] Pawlow P. Z. Phys. Chem., 1909, 65, 545

[35] Reiss H.; Mirabel P.; Whetten R.L. J. Phys. Chem., 1988, 92, 7241.

[36] Peters K.F.; Chung Y.W.; Cohen J.B. Appl. Phys. Lett., 1997, 71, 20.

[37] Couchmann P.R.; Jesser W.A. Nature, 1977, 269, 481

[38] Vanfleet R.R.; Mochel J.M. Surf. Sci., 1995, 341, 40.

[39] Peters K. F.; Cohen J.B.; Chung Y.W. Phys. Rev. B, 1998, 57, 13430.

[40] Jones D.R.H. J. Mater. Sci., 1974, 9, 1.

[41] Jiang Q.; Shi H.X.; Zhao M. Acta Mater., 1999, 47, 2109.

[42] Peters K.F.; Cohen J.B.; Chung Y.W. Phys. Rev. B, 1998, 57, 13430.

[43] Sun C.Q. Prog. Mater. Sci., 2003, 48, 521.

[44] Sun C.Q.; Li C.M.; Bai H.L.; Jiang E.Y. Nanotechnology, 2005, $16,1290$.

[45] Sun C.Q.; Shi Y.; Li C.M.; Li S.; Au Yeung T.C. Phys. Rev. B, 2006, 73, 075408

[46] Shi F.G. J. Mater. Res., 1994, 9, 1307.

[47] Sun C.Q.; Chen T.P.; Tay B.K.; Li S.; Huang H.; Zhang Y.B.; Pan L.K.; Lau S.P.; Sun X.W. J. Phys. D: Appl. Phys., 2001, 34, 3470.

[48] Lang X.Y.; Jiang Q. Solid State Commun., 2005, 134, 797.

[49] Lindemann F.A. Z. Phys., 1910, 11, 609.

[50] Hoshino K.; Shimamura S. Phil. Mag. A, 1979, 40, 137.

[51] Dash J.G. Rev. Mod. Phys., 1999, 71, 1737.

[52] Jiang Q.; Aya N.; Shi F.G. Appl. Phys. A, 1997, 64, 627.

[53] Mott N.F. Proc. R. Soc. A, 1934, 146, 465.

[54] Regel' A.R.; Glazov V. M. Semiconductors, 1995, 29, 405.

[55] Tiwari G.P.; Juneja J.M.; Iijima Y. J. Mater. Sci., 2004, 39, 1535.

[56] Buckwald R.A.; Hirsch A.A.; Cabib D.; Callen E. Phys. Rev. Lett., $\mathbf{1 9 7 5}, 35,878$.

[57] http://www.webelements.com/.

[58] Yang C.C.; Xiao M.X.; Li W.; Jiang Q. Solid State Commun., 2006, 139, 148.

[59] Meyers M.A.; Mishra A.; Menson D. J. Prog. Mater. Sci., 2006, $51,427$.

[60] Arzt E.; Ashby M.F. Script. Met., 1982, 16, 1285.

[61] Zhao M.; Li J.C.; Jiang Q. J. Alloy Comp., 2003, 361, 160.

[62] Lu K.; Sui M.L. Script Met., 1993, 28, 1465.

[63] Jang J.S.C.; Koch C.C. Script. Metal. Mater., 1990, 24, 1599

[64] Fu H.H.; Benson D.J.; Meyers M.A. Acta Mater., 2001, 49, 2567.

[65] El-Sherik A.M.; Erb U.; Palumbo G.; Aust K.T. Script. Metal Mater., 1992, 27, 1185.

[66] Kim H.S. Script. Mater., 1998, 39, 1057.

[67] Schuh C.A.; Nieh T.G.; Yamasaki T. Script. Mater., 2002, 46, 735.

[68] Streitz F.H.; Cammarata R.C.; Sieradzki K. Phys. Rev. B, 1994, 49, 10699.

[69] Dodson B.W. Phys. Rev. Lett., 1988, 60, 2288.

[70] Hall E.O. Proc. Phys. Soc. B, 1951, 64, 747.

[71] Petch N.J. J. Iron Steel Inst. 1953, 174, 25.

[72] Sun C.Q.; Li S.; Li C.M. J. Phys. Chem. B, 2005, 109, 415

[73] Conrad H.; Narayan J. Script. Mater., 2000, 42, 1025.

[74] Masumura R.A.; Hazzledine P.M.; Pande C.S. Acta Mater., 1998, $46,4527$.

[75] Qin X.Y.; Zhu X.G.; Gao S.; Chi L.F.; Lee J.S. J. Phys. Condens. Matter, 2002, 14, 2605.

[76] Kocks U.F.; Argon A.S.; Ashby A.S. Prog. Mater. Sci., 1975, 19, 1.

[77] Jiang Q.; Zhang S.H.; Li J.C. Solid. State Commun., 2004, 130, 581 .

[78] Liang L.H.; Shen G.M.; Chen X.P.; Liu W.M.; Gao H.J. J. Phys. Condens. Matter, 2004, 16, 267.

[79] Yamakov V.; Wolf D.; Phillpot S.R.; Mukherjee A.K.; Gleiter H. Nat. Mater., 2002, 1, 45

[80] Takeuchi S. Script. Mater., 2001, 44, 1483.

[81] Schiøtz J.; Di Tolla F.D.; Jacobsen K.W. Nature, 1998, 391, 561.
[82] Yip S. Nature, 1998, 391, 532.

[83] Jungwirth T.; Sinova J.; Mašek J.; Kučera J. Rev. Mod. Phys., 2006, 78, 809 .

[84] von Barth U.; Hedin L. J. Phys. C, 1972, 5, 1629.

[85] van Vleck J.H. Rev. Mod. Phys., 1945, 17, 27.

[86] Kittel C. Rev. Mod. Phys., 1949, 21, 541.

[87] Jensen P.J.; Benemann K.H. Surf. Sci. Rep., 2006, 61, 129.

[88] Buschow K.H.J.; de Boer F.R. Physics of Magnetism and Magnetic Materials, Kluwer Acad. Pub. (New York), 2003.

[89] Slater J.C. Phys. Rev., 1930, 35, 509.

[90] Slater J.C. Phys. Rev., 1930, 36, 57.

[91] Sommerfeld A.; Bethe H. in Geiger H.; Schell K. (Eds) Handbook of Physics, Berlin: Springer 1936, 24, 595.

[92] Poulopoulos P.; Baberschke K. J. Phys.: Condens. Matter, 1999, 11,9495 .

[93] Lee E.L.; Bolduc P.E.; Violet C.E. Phys. Rev. Lett., 1964, 13, 800.

[94] Stampanoni M.; Vaterlaus A.; Aeschlimann M.; Meier F. Phys. Rev. Lett., 1987, 59, 2483.

[95] Dürr W.; Taborelli M.; Paul O.; Germar R.; Gudat W.; Pescia D.; Landolt M. Phys. Rev. Lett., 1989, 62, 206.

[96] Liu C.; Bader S.D. J. Appl. Phys., 1990, 67, 5758

[97] Qiu Z.Q.; Pearson J.; Bader S.D. Phys. Rev. Lett., 1991, 67, 1646.

[98] Qiu Z.Q.; Pearson J.; Bader S.D. Phys. Rev. Lett., 1993, 70, 1006.

[99] Schneider C.M.; Bressler P.; Schuster P.; Kirschner J. Phys. Rev. Lett., 1990, 64, 1059.

[100] Huang F.; Mankey G.J.; Kief M.T.; Willis R.F. J. Appl. Phys., 1993, 73, 6760 .

[101] Huang F.; Kief M.T.; Mankey G.J.; Willis R.F. Phys. Rev. B, 1994, 49, 3962.

[102] May F.; Srivastava P.; Farle M.; Bovensiepen U.; Wende H.; Chauvistré R.; Baberschke K. J. Magn. Magn. Mater., 1998, 177$181,1220$.

[103] Ballentine C.A.; Fink R.L.; Araya-Pochet J.; Erskine J.L. Phys. Rev. B, 1990, 41, 2631.

[104] Li Y.; Baberschke K. Phys. Rev. Lett., 1992, 68, 1208.

[105] Farle M.; Baberschke K.; Stetter U.; Aspelmeier A.; Gerhardter F. Phys. Rev. B, 1993, 47, 11571.

[106] Jiang J.S.; Chien C.L. J. Appl. Phys., 1996, 79, 5615.

[107] Jiang J.S.; Davidović D.; Reich D.H.; Chien C.L. Phys. Rev. Lett., 1995, $74,314$.

[108] Gajdzik M.; Trappmann T.; Sürgers C.; Lőhneysen H.V. Phys. Rev. $B, \mathbf{1 9 9 8}, 57,3525$.

[109] Sun L.; Searon P.C.; Chien C.L. Phys. Rev. B, 2000, 61, R6463.

[110] Cui X.F.; Zhao M.; Jiang Q. Thin Solid Films, 2005, 472, 328.

[111] Du Y.W.; Xu M.X.; Wu J.; Shi Y.B.; Lu H.X.; Xue R.H. J. Appl. Phys., 1991, 70, 5903.

[112] Michels D.; Krill III C.E.; Birringer R. J. Magn. Magn. Mater., 2002, 250, 203.

[113] Sun C.Q.; Zhong W.H.; Li S.; Tay B.K.; Bi H.L.; Jiang E.Y. J. Phys. Chem. B, 2004, 108, 1080

[114] Tang Z.X.; Sorensen C.M.; Klabunde K.J.; Hadjipanayis G.C. Phys. Rev. Lett., 1991, 67, 3602.

[115] Hernando A.; Navarro I.; Prados C.; García D.; Vázquez M.; Alonso J. Phys. Rev. B, 1996, 53, 8223.

[116] Skomski R.; Coey J.M. Phys. Rev. B, 1993, 48, 812.

[117] Hernando A. J. Magn. Magn. Mater., 1992, 117, 154.

[118] Grünberg P.; Schreiber R.; Pang Y.; Brodsky M.B.; Sowers H. Phys. Rev. Lett., 1986, 57, 2442.

[119] Parkin S.S.P.; More N.; Roche K.P. Phys. Rev. Lett., 1990, 64, 2340.

[120] Fisher M.E.; Barber M.N. Phys. Rev. Lett., 1972, 28, 1516.

[121] Zhang R.J.; Willis R.F. Phys. Rev. Lett., 2001, 86, 2665.

[122] Nikolaev V.I.; Shipilin A.M. Phys. Solid State, 2003, 45, 1079.

[123] Yang C.C.; Jiang Q. Acta Mater., 2005, 53, 3305.

[124] Lang X.Y.; Zheng W.T.; Jiang Q. Phys. Rev. B, 2006, 73, 224444.

[125] Aguilera-Granja F.; Morán-López J.L. Solid State Commun., 1990, 74,155 .

[126] van der Zaag P.J.; Ball A.R.; Feiner L.F.; Wolf R.M.; van der Heijden P.A.A. J. Appl. Phys., 1996, 79, 5103.

[127] Jiang Q.; Lang X.Y., Macromol. Rapid Commun., 2004, 25, 825.

[128] Weschke E.; Ott H.; Schierle E.; Schüßler-Langeheine C.; Vyalikh D.V.; Kaindl G.; Leiner V.; Ay M.; Schmitte T.; Zabel H.; Jensen P.J. Phys. Rev. Lett., 2004, 93, 157204.

[129] Galanakis I.; Papanikolaou N.; Dederichs P.H. Surf. Sci., 2002, 511,1 . 
[130] Jiang Q.; Lu H.M.; Zhao M. J. Phys. Condens. Matter, 2004, 16, 521.

[131] Johnson D.; Perera P.; O`Shea M.J. J. Appl. Phys., 1996, 79, 5299.

[132] Chen J.P.; Sorensen C.M.; Klabunde K.J.; Hadjipanayis G.C. Phys. Rev. B, 1995, 55, 11527.

[133] Cox D.M.; Trevor D.J.; Whetten R.L.; Rohlfing E.A.; Kaldor A. Phys. Rev. B, 1985, 32, 7290.

[134] Zhong W.H. Sun C.Q.; Li S.; Bai H.L.; Jiang E.Y. Acta Mater., 2005, 53, 3207.

[135] Pauling L. J. Am. Chem. Soc., 1947, 69, 542.

[136] Goldschmidt V.M. Ber. Deut. Chem. Ges., 1972, 60, 1270.

[137] Billas I.M.L.; Chatelain A.; de Heer W.A. Science, 1994, 265, 1682.

[138] Konno M. J. Phys. Soc. Jpn., 1980, 49, 1185.

[139] Liu F.; Press M.R.; Khanna S.N.; Jena P. Phys. Rev. B, 1989, 39, 6914.

[140] Kodama R.H. J. Magn. Magn. Mater., 1999, 200, 359.

[141] Kodama R.H.; Berkowitz A.E.; Mcniff E.J.; Foner S. Phys. Rev. Lett., 1996, 77, 394.

[142] Kodama R.H.; Berkowitz A.E. Phys. Rev. B, 1999, 59, 6321.

[143] Kodama R.H.; Berkowitz A.E.; Mcniff E.J.; Foner, S. J. Appl. Phys., 1997, 81, 5552.

[144] Sumiyama K.; Sato T.; Graham G.M. Solid State Commun., 1976, $19,403$.

[145] Wedler G.; Schneck H. Thin Solid Films, 1977, 47, 147.

[146] Lu H.M.; Zheng W.T.; Jiang Q. J. Phys. D: Appl. Phys., 2007, 40, 320.

[147] Gong W.; Li H.; Zhao Z.R.; Chen J.C. J. Appl. Phys., 1991, 69, 5119.

[148] Berkowitz A.E.; Shuele W.J.; Flanders P.J. J. Appl. Phys., 1968, 39, 1261.

[149] Panda R.N.; Gajbhiye N.S.; Balaji G. J. Alloy Compd., 2001, 326, 50.

[150] Liu Z.L.; Liu Y.J.; Yao K.L.; Ding Z.H.; Tao J.; Wang X. J. Mater. Synth. Process., 2002, 10, 83.

[151] Amulevicius A.; Baltrunas D.; Bendikiene V.; Daugvila A.; Davidonis D.; Mazeika K. Phys. Stat. Sol. A, 2002, 189, 243.

[152] Grigorova M.; Blythe H.J.; Blaskov V.; Rusanov V.; Petkov V.; Masheva V.; Nihtianova D.; Martinze L.M.; Munoz J.S.; Mikhov M. J. Magn. Magn. Mater., 1998, 183, 163.

[153] Rajendran M.; Pullar R.C.; Bhattacharya A.K.; Das D.; Chintalapudi S.N.; Majumdar C.K. J. Magn. Magn. Mater., 2001, 232, 71.

[154] Tang Z.X.; Sorensen C.M.; Klabunde K.J.; Hadjipanayis G.C. J. Appl. Phys., 1991, 69, 5279.

[155] Chen J.P.; Sorensen C.M.; Klabunde K.J.; Hadjipanayis G.C.; Devlin E.; Kostikas A. Phys. Rev. B, 1996, 54, 9288.

[156] Coey J.M.D. Phys. Rev. Lett., 1971, 27, 1140.

[157] Okada T.; Sekizawa H.; Ambe F.; Ambe S.; Yamadaya T. J. Magn. Magn. Mater., 1983, 31-34, 903.

[158] Parker F.T.; Foster M.W.; Margulies D.T.; Berkowitz A.E. Phys. Rev. B, 1993, 47, 7885 .

[159] Martínez B.; Obradors X.; Balcells L.; Rouanet A.; Monty C. Phys. Rev. Lett., 1998, 80, 181.

[160] Kodama R.H.; Berkowitz A.E.; McNiff Jr. E.J.; Foner S. Phys. Rev. Lett., 1996, 77, 394.

[161] Mamiya H.; Nakatani I.; Furubayashi T. Phys. Rev. Lett., 1998, 80, 177.

[162] Zhong W.H.; Sun C.Q.; Li S. Solid State Commun., 2004, 130, 603.

[163] Johnson M.T.; Bloemen P.J.H.; den Broeder F.J.A.; de Vries J.J. Rep. Prog. Phys., 1996, 59, 1409.

[164] van der Merwe J.H. J. Appl. Phys., 1963, 34, 123.

[165] Li J.C.; Liu W.; Jiang Q. Appl. Surf. Sci., 2005, 239, 259.

[166] Chappert C.; Bruno P. J. Appl. Phys., 1988, 64, 5736.

[167] Fritzsche H.; Kohlhepp J.; Elmers H.J.; Gradmann U. Phys. Rev. B, 1994, 49, 15665.

[168] Araya-Pochet J.; Ballentine C.A.; Erskine J.L. Phys. Rev. B, 1988, 38, 7846.

[169] Schulz B.; Baberschke K. Phys. Rev. B, 1994, 50, 13467.

[170] Gerhardter F.; Li Y.; Baberschke K. Phys. Rev. B, 1993, 47, 11204.

[171] Prokop J.; Valdaitsev D.A.; Kukunin A.; Pratzer M.; Schönhens G.; Elmers H.J. Phys. Rev. B, 2004, 70, 184423.

[172] Heinrich B.; Purcell S.T.; Dutcher J.R.; Urquhart K.B.; Cochran J.F.; Arrott A.S. Phys. Rev. B, 1988, 38, 12879 .

[173] Kawakami R.K.; Escorcia-Aparicio E.J.; Qiu Z.Q. Phys. Rev. Lett., 1996, 77, 2570 .
[174] Bisio F.; Moroni R.; de Mongeot F.B.; Canepa M.; Mattera L. Phys. Rev. Lett., 2006, 96, 057204.

[175] Chen J.; Erskine J.L. Phys. Rev. Lett., 1992, 68, 1212.

[176] Choi H.J.; Qiu Z.Q.; Pearson J.; Jiang J.S.; Li D.; Bader S.D. Phys. Rev. B, 1998, 57, R12713.

[177] Berger A.; Linke U.; Oepen H.P. Phys. Rev. Lett., 1992, 68, 839.

[178] Chuang D.S.; Ballentine C.A.; O'Handley R.C. Phys. Rev. B, 1994, 49, 15084.

[179] Farle M.; Mirwald-Schulz B.; Anisimov A.N.; Platow W.; Baberschke K. Phys. Rev. B, 1997, 55, 3708.

[180] Anisimov A.N.; Farle M.; Poulopoulos P.; Platow W.; Baberschke K.; Isberg P.; Wäppling R.; Niklasson A.M.N.; Eriksson O. Phys. Rev. Lett., 1999, 82, 2390.

[181] Geldart D.J.W.; Hargraves P.; Fujiki N.M.; Dunlap R.A. Phys. Rev. Lett., 1989, 62, 2728.

[182] Gradmann U.; Müller J. Phys. Status Solidi B, 1968, 27, 313.

[183] Thomassen J.; May F.; Feldmann B.; Wuttig M.; Ibach H. Phys. Rev. Lett., 1992, 69, 3831.

[184] Liu C.; Moog E.R.; Bader S.D. Phys. Rev. Lett., 1988, 60, 2422.

[185] Castrucci P.; Gunnella R.; Bernardini R.; Falcioni P.; de Crescenzi M., Phys. Rev. B, 2002, 65, 235435.

[186] Peterka D.; Enders A.; Haas G.; Kern K. Phys. Rev. B, 2002, 66, 104411.

[187] Enders A.; Peterka D.; Repetto D.; Lin N.; Dmitriev A.; Kern K. Phys. Rev. Lett., 2003, 90, 217203.

[188] Berger A.; Hopster H. Phys. Rev. Lett., 1996, 76, 519.

[189] Garreau G.; Hajjar S.; Bubendorff J.L.; Pirri C.; Berling D.; Mehdaoui A.; Stephan R.; Wetzel P.; Zabrocki S.; Gewinner G. Phys. Rev. B, 2005, 71, 094430.

[190] Oepen H.P.; Speckmann M.; Millev Y.; Kirschner J. Phys. Rev. B, 1997, 55, 2752.

[191] Kisielewski M.; Maziewski A.; Tekielak M.; Wawro A.; Baczewski L.T. Phys. Rev. Lett., 2002, 89, 087203.

[192] Lee J.W.; Jeong J.R.; Shin S.C.; Kim J.; Kim S.K. Phys. Rev. B, 2002, 66, 172409.

[193] Sellmann R.; Fritzsche H.; Maletta H.; Leiner V.; Siebrecht R. Phys. Rev. B, 2001, 63, 224415.

[194] Zhao H.W.; Wu Y.Z.; Won C.; Toyoma F.; Qiu Z.Q. Phys. Rev. B, 2002, 66, 104402.

[195] Sander D.; Pan W.; Ouazi S.; Kirschner J.; Meyer W.; Krause M.; Müller S.; Hammer L.; Heinz K. Phys. Rev. Lett., 2004, 93, 247203.

[196] Hucht A.; Usadel K.D. Phys. Rev. B, 1997, 55, 12309.

[197] Hucht A.; Usadel K.D. J. Magn. Magn. Mater., 1999, 198-199, 493.

[198] Baberschke K.; Farle M. J. Appl. Phys., 1997, 81, 5038.

[199] Kittel C. Phys. Rev., 1946, 70, 965.

[200] Néel L. Comptes Rendus (Paris) 1947, 224, 1550.

[201] Stoner E.C.; Wohlfarth E.P. Nature, 1947, 160, 650.

[202] Leslie-Pelecky D.L.; Rieke R.D. Chem. Mater., 1996, 8, 1770.

[203] Gong W.; Li H.; Zhao Z.; Chen J. J. Appl. Phys., 1991, 69, 5119.

[204] Kittel C. Phys. Rev., 1948, 73, 810.

[205] Li Z.W.; Lin G.Q.; Chen L.; Wu Y.P.; Ong C.K. J. Appl. Phys., 2005, $98,094310$.

[206] Chien C.L. Annu. Rev. Mater. Sci., 1995, 25, 129.

[207] Alben R.; Becker J.J.; Chi M.C. J. Appl. Phys., 1978, 49, 1653.

[208] Kneller E.F.;. Luborsky F.E. J. Appl. Phys., 1963, 34, 656.

[209] Sawa T.; Takahashi Y. J. Appl. Phys., 1990, 67, 5565.

[210] Herzer G. J. Magn. Magn. Mater., 1992, 112, 258.

[211] Dai J.B.; Malkinski L.M.; Tang J.K. IEEE Trans. Magn., 2000, 36, 2918.

[212] Abeles B.; Sheng P.; Counts M.D.; Arie Y. Adv. Phys., 1975, 24, 407.

[213] Chien C.L. J. Appl. Phys., 1991, 69, 5627.

[214] Xiao G.; Liou S.H.; Levy A.; Taylor J.N.; Chien C.L. Phys. Rev. B, 1986, 34, 7573

[215] Morrish A.H. Physical Principle of Magnetism, New York: Wiley, 1965.

[216] Dormann J.L.; Fiorani D.; Tholence J.L.; Sella C. J. Magn. Magn. Mater., 1983, 35, 117.

[217] Childress J.R., Chien C.L.; Rhyne J.J.; Erwin R.W. J. Magn. Magn. Mater., 1992, 104-107, 1585.

[218] Frolov G.I. Tech. Phys., 2004, 49, 909.

[219] Barker B.; Lemmens L.F. Phys. Rev. E, 2005, 71, 046109.

[220] Dudzik E.; Drr H.A.; Dhesi S.S.; van der Laan G.; Knabben D.; Goedkoop J.B. J. Phys. Condens. Matter, 1999, 11, 8445. 
[221] Liu C.; Zou B.S.; Rondinone A.J.; Zhang Z.J. J. Am. Chem. Soc., 2000, 122, 6263.

[222] Rondinone A.J.; Samia A.C.S.; Zhang Z.J. J. Phys. Chem. B, 1999, $103,6876$.

[223] Liu C.; Zou B.S.; Rondinone A.J.; Zhang Z.J. J. Phys. Chem. B, 2000, 104, 1141.

[224] Liu C.; Zhang Z.J. Chem. Mater., 2001, 13, 2092

[225] Han M.; Vestal C.R.; Zhang Z.J. J. Phys. Chem. B, 2004, 108, 583.

[226] Kale A.; Nathani H.; Srivastava R.S.; Misra R.D.K. Mater. Sci. Tech., 2004, 20, 999.

[227] Jeong U.; Teng X.W.; Wang Y.; Yang H.; Xia Y.N. Adv. Mater., 2007, 19, 33.

[228] Bean C.P.; Jacobs I.S. J. Appl. Phys., 1956, 27, 1448.

[229] Néel L.; Hebd C.R. Seances Acad. Sci. 1961, 252, 4075; 1961, 253 , 9; 1961, 253, 1286.

[230] Lederman D.; Ramos C.A.; Jaccarino V.; Cardy J.L. Phys. Rev. B, 1993, 48, 8365 .

[231] Lederman D.; Ramos C.A.; Jaccarino V. J. Phys.: Condens. Matter, 1993, 5, A373.

[232] Ambrose T.; Chien C.L. Phys. Rev. Lett., 1996, 76, 1743.

[233] Tang Y.J.; Smith D.J.; Zink B.L.; Hellman F.; Berkowitz A.E. Phys. Rev. B, 2003, 67, 054408.

[234] Abarra E.N.; Takano K.; Hellman F.; Berkowitz A.E. Phys. Rev. Lett., 1996, 77, 3451.

[235] Alders D.; Tjeng L.H.; Voogt F.C.; Hibma T.; Sawatzky G.A.; Chen C.T.; Vogel J.; Sacchi M.; Iacobucci S. Phys. Rev. B, 1998, 57, 11623.

[236] Makhlouf S.A.; Parker F.T.; Spada F.E.; Berkowitz A.E. J. Appl. Phys., 1997, 81, 5561.

[237] Sako S.; Ohshima K.; Sakai M.; Bandow S. Surf. Rev. Lett., 1996, 3, 109.

[238] Sako S.; Ohshima K. J. Phys. Soc. Jpn., 1995, 64, 944.

[239] Punnoose A.; Magnone H.; Seehra M.S.; Bonevich J. Phys. Rev. B, 2001, 64, 174420.

[240] Punnoose A.; Seehra M.S. J. Appl. Phys., 2002, 91, 7766.

[241] Stewart S.J.; Multigner M.; Marco J.F.; Berry F.J.; Hemando A.; Gonzalez J.M. Solid State Commun., 2004, 130, 247.

[242] Zheng X.G.; Xu C.N.; Nishikubo K.; Nishiyama K.; Higemoto W.; Moon W.J.; Tanaka E.; Otabe E.S. Phys. Rev. B, 2005, 72, 014464.

[243] Ledermann D.; Ramos C.A.; Jaccarino V. J. Phys. Condens. Matter, 1993, 5, A373.

[244] Ledermann D.; Ramos C.A.; Jaccarino V. Phys. Rev. B, 1993, 48, 8365.

[245] Carey M.J.; Berkowitz A.E.; Borchers J.A.; Erwin R.W. Phys. Rev. $B, \mathbf{1 9 9 3}, 47,9952$.

[246] Borchers J.A.; Carery M.J.; Erwin R.W.; Majkrzak C.F.; Berkowitz A.E. Phys. Rev. Lett., 1993, 70, 1878.

[247] Carriço A.S.; Camley R.E. Phys. Rev. B, 1992, 45, 13117.

[248] Zhang S.F.; Zhang G.H. J. Appl. Phys., 1994, 75, 6685.

[249] Ramos C.A.; Lederman D.; King A.R.; Jaccarino V. Phys. Rev. Lett., 1990, 65, 2913.

[250] Takano M.; Terashima T.; Bando Y.; Ikeda H. Appl. Phys. Lett., 1987, 51, 205

[251] van der Zaag P.J.; Ijiri Y.; Borchers J.A.; Feiner L.F.; Wolf R.M.; Gaines J.M.; Erwin R.W.; Verheijen M.A. Phys. Rev. Lett., 2000, $84,6102$.

[252] Fullerton E.E.; Riggs K.T.; Sowers C.H.; Bader S.D. Phys. Rev. Lett., 1995, 75, 330.

[253] Fullerton E.E.; Adenwalla S.; Felcher G.P.; Riggs K.T.; Sowers C.H.; Bader S.D.; Robertson J.L., Physica B, 1996, 221, 370.

[254] Fawcett E. Rev. Mod. Phys., 1988, 60, 209.

[255] Nicklow R.M.; Mook H.A.; Smith H.G.; Reed R.E.; Wilkinson M.K. J. Appl. Phys., 1969, 40, 1452.

[256] Bohr J.; Gibbs D.; Axe J.D.; Moncton D.E.; D'amico K.L.; Majkrzak C.F.; Kwo J.; Hong M.; Chien C.L.; Jensen J. Physica B, 1989, 159, 93.

[257] Hernando A. J. Phys. Condens. Matter, 1999, 11, 9455.

[258] Jiang Q.; Tong H.Y.; Hsu D.T.; Okuyama K.; Shi F.G. Thin Solid Films, 1998, 312, 357.

[259] Shull C.G.; Strauser W.A.; Wollan E.O. Phys. Rev., 1951, 83, 333.

[260] Artman J.O.; Murphy J.C.; Foner S. Phys. Rev., 1965, 138, A912.

[261] Nagai O.; Bonavito N.L.; Tanaka T. J. Phys. C: Solid State Phys., 1973, 6, L470.

[262] Gcallagher P.K.; Gyorgy E.M. Phys. Rev., 1969, 180, 622.

[263] Yamamoto N. J. Phys. Soc. Jpn., 1968, 24, 23.

[264] Amin N.; Arajs S. Phys. Rev. B, 1987, 35, 4810.
[265] Bødker F.; Hansen M.F.; Koch C.B.; Lefmann K.; Mørup S. Phys. Rev. B, 2000, 61, 6826.

[266] Liu L.; Kou H.Z.; Mo W.L.; Liu H.J.; Wang Y.Q. J. Phys. Chem. $B, 2006,110,15218$.

[267] Zysler R.D.; Fiorani D.; Testa A.M.; Godinho M.; Agostinelli E.; Suber L. J. Magn. Magn. Mater., 2004, 272-276, 1575.

[268] Zysler R.D.; Fiorani D.; Testa A.M.; Suber L.; Agostinelli E.; Godinho M. Phys. Rev. B, 2003, 68, 212408.

[269] Ayyub P.; Multani M.; Barma M.; Palkar V.R.; Vijayaraghavan R. J. Phys. C: Solid State Phys., 1988, 21, 2229.

[270] Nininger R.C.; Schroeer D. J. Phys. Chem. Solids, 1978, 39, 137.

[271] Wayne R.C.; Anderson D.H. Phys. Rev., 1967, 155, 496.

[272] Searle C.W. Phys. Lett. A, 1967, 25, 256.

[273] Muench G.J.; Arajs S.; Matijevic E. Phys. Status Solid A, 1985, 92, 187.

[274] Kündig W.; Bömmel H.; Constabaris G.; Lindquist R.H. Phys. Rev., 1966, 142, 327

[275] Dang M.Z.; Rancourt D.G.; Dutrizac J.E.; Lamarche G.; Provencher R. Hyperfine Interact., 1998, 117, 271.

[276] Stamps R.L. J. Phys. D Appl. Phys., 2000, 33, R247.

[277] Meiklejohn W.H.; Bean C.P. Phys. Rev., 1956, 102, 1413; 1957, 105,904

[278] Meiklejohn W.H. J. Appl. Phys., 1962, 33, 1328.

[279] Lin H.M.; Hsu C.M.; Yao Y.D.; Cben Y.Y.; Kuan T.T.; Yang F.A.; Tung C.Y. Nanostruct. Mater., 1995, 6, 977.

[280] Gangopadhyay S.; Hadjipanayis G.C.; Sorensen C.M.; Klabunde K.J. Nanostruct. Mater., 1992, 1, 449.

[281] Löffler J.; Van Swygenhoven H.; Wagner W.; Meier J.; Doudin B.; Ansermet J.Ph. Nanostruct. Mater. 1997, 9, 523.

[282] Yao Y.D.; Chen Y.Y.; Hsu C.M.; Lin H.M.; Tung C.Y.; Tai M.F.; Wang D.H.; Wu K.T.; Suo C.T. Nanostruct. Mater., 1995, 6, 933.

[283] Meiklejohn W.H. J. Appl. Phys., 1958, 29, 454

[284] Darnell F.J. J. Appl. Phys., 1961, 32,186S.

[285] Papaefthymiou V.; Kostikas A.; Simopoulos A.; Niarchos D.; Gangopadyay S.; Hadjipanayis G.C.; Sorensen C.M.; Klabunde K.J. J. Appl. Phys., 1990, 67, 4487.

[286] Moran T.J.; J Gallego.M.; Schuller I.K. J. Appl. Phys., 1995, 78, 1887.

[287] Berkowitz A.E.; Greiner J.H. J. Appl. Phys., 1965, 36, 3330.

[288] Lang X.Y.; Zheng W.T.; Jiang Q. Nanotechnology, 2007, 18, 155701.

[289] Papaefthymiou V.; Kostikas A.; Simopoulos A.; Niarchos D.; Gangopadyay S.; Hadjipanayis G.C.; Sorensen C.M.; Klabunde K.J. J. Appl. Phys., 1990, 67, 4487.

[290] Tsang C. J. Appl. Phys., 1984, 55, 2226.

[291] Dieny B.; Speriosu V.S.; Parkin S.S.P.; Gurney B.A.; Wilhoit D.R.; Mauri D. Phys. Rev. B, 1991, 43, 1297.

[292] Gurney B.A.; Speriosu V.S.; Wihoit D.R.; Lefakis H.; Fontana R.E.; Heim D.E.; Dovek M.; J. Appl. Phys., 1997, 81, 3998.

[293] Nogués J.; Schuller I.K. J. Magn. Magn. Mater., 1999, 192, 203.

[294] Berkowitz A.E.; Takano K. J. Magn. Magn. Mater., 1999, 200, 552.

[295] Kiwi M. J. Magn. Magn. Mater., 2001, 234, 584.

[296] Ali M.; Adie P.; Marrows C.H.; Greig D.; Hickey B.J.; Stamps R.L. Nat. Mater., 2007, 6, 70.

[297] Nogués J.; Lederman D.; Moran T.J.; Schuller I.K. Phys. Rev. Lett., 1996, 76, 4624.

[298] Jungblut R.; Coehoorn R.; Johnson M.T.; aan de Stegge J.; Reinders A. J. Appl. Phys., 1994, 75,6659.

[299] Nogués J.; Moran T.J.; Lederman D.; Schuller I K.; Rao K.V. Phys. Rev. B, 1999, 59, 6984.

[300] van der Zaag P.J.; Ball A.R.; Feiner L.F.; Wold R.M.; van der Heijden P.A.A. J. Appl. Phys., 1996, 79, 5103.

[301] Shen J.X.; Kief M.T. J. Appl. Phys., 1996, 79, 5008.

[302] Park C.M.; Min K.I.; Shin K.H. J. Appl. Phys., 1996, 79, 6228.

[303] Lee S.S.; Hwang D.G.; Park C.M.; Lee K.A.; Rhee J.R. J. Appl. Phys., 1997, 81, 5298.

[304] Lederman D.; Nogués J.; Schuller I.K. Phys. Rev. B, 1997, 56, 2332.

[305] Nogués J.; Lederman D.; Moran T.J.; Schuller I. K.; Rao K.V. Appl. Phys. Lett., 1996, 68, 3186.

[306] Soeya S.; Fuyama M.; Tadokoro S.; Imagawa T. J. Appl. Phys., 1996, 79, 1604.

[307] Wong B.Y.; Mitsumata C.; Prakash S.; Laughlin D.E.; Kobayashi T. J. Appl. Phys., 1996, 79, 7896. 
[308] Fujiwara H.; Nishioka K.; Hou C.; Parker M.R.; Gangopadhyay S.; Metzger R. J. Appl. Phys., 1996, 79, 6586.

[309] Uyama H.; Otani Y.; Fukamichi K.; Kitakami O.; Shimada Y.; Echigoya J. Appl. Phys. Lett., 1997, 71, 1258.

[310] Takano K.; Kodama R.H.; Berkowitz A.E.; Cao W.; Thomas G. Phys. Rev. Lett., 1997, 79, 1130.

[311] Chopra H.S.; Hockey B.J.; Chen P.J.; McMichael R.D.; Egelhoff Jr. W.F. J. Appl. Phys., 1997, 81, 4017.

[312] Fulcomer E.; Charap S.H. J. Appl. Phys., 1972, 43, 4184.

[313] Tsang C.; Lee K. J. Appl. Phys., 1982, 53, 2605.

[314] Lai C.H.; Matsuyama H.; White R.L.; Anthony T.C.; G Bush.G. J. Appl. Phys., 1996, 79, 6389.

[315] Tsunoda M.; Tsuchiya Y.; Konoto M.; Takahashi M. J. Magn. Magn. Mater., 1997 171, 29.

[316] Néel L. Ann. Phys. (Paris) 1967, 2, 61

[317] Mauri D.; Siegmann H.C.; Bagus P.S.; Kay E. J. Appl. Phys., 1987, $62,3047$.

[318] Koon N.C. Phys. Rev. Lett., 1997, 78, 4865.

[319] Schulthess T.C.; Butler W.H. Phys. Rev. Lett., 1998, 81, 4516.

[320] Schulthess T.C.; Butler W.H. J. Appl. Phys., 1999, 85, 5510.

[321] Malozemoff A.P. Phys. Rev. B, 1987, 35, 3679.

[322] Malozemoff A.P. Phys. Rev. B, 1988, 37, 7673.

[323] Malozemoff A.P. J. Appl. Phys., 1988, 63, 3874.

[324] Ijiri Y.; Borchers J.A.; Erwin R.W.; Lee S.H.; van der Zaag P.J.; Wolf R.M. Phys. Rev. Lett., 1998, 80, 608.
[325] Devasahayam A.J.; Kryder M.H. J. Appl. Phys., 1999, 85, 5519.

[326] Baltz V.; Sort J.; Landis S.; Rodmacq B.; Dieny B. Phys. Rev. Lett., $\mathbf{2 0 0 5}, 94,117201$

[327] Xi H.W.; White R.M.; Gao Z.; Mao S.N. J. Appl. Phys., 2002, 92, 4828.

[328] Fuke H.N.; Saito K.; Yoshikawa M.; Iwasaki H.; Sahashi M. Appl. Phys. Lett., 1999, 75, 3680.

[329] van Driel J.; de Boer F.R.; Lenssen K.M.H.; Coehoorn R. J. Appl. Phys., 2000, 88, 975.

[330] Sang H.; Du Y.W.; Chien C.L. J. Appl. Phys., 1999, 85, 4931.

[331] Rickart M.; Guedes A.; Ventura J.; Sousa J.B.; Freitas P.P. J. Appl. Phys., 2005, 97, 10K110.

[332] Lund M.S.; Macedo W.A.A.; Liu K.; Nogués J.; Schuller I.K.; Leighton C. Phys. Rev. B, 2002, 66, 054422.

[333] Fulcomer E.; Charap S.H. J. Appl. Phys., 1972, 43, 4190.

[334] Xi H.W.; White R.M. J. Appl. Phys., 2003, 94, 5850.

[335] Ambrose T.; Chien C.L. J. Appl. Phys., 1996, 79, 5920.

[336] Ali M.; Marrows C.H.; Al-Jawad M.; Hickey B.J.; Misra A.; Nowak U.; Usadel K.D. Phys. Rev. B, 2003, 68, 214420.

[337] Jungblut R.; Coehoorn R.; Johnson M.T.; aan de Stegge J.; Reinders A. J. Appl. Phys., 1994, 75, 6659.

[338] Scholl A.; Liberati M.; Arenbolz E.; Ohldag H.; Sthör J.; Phys. Rev. Lett., 2004, 92, 247201.

[339] Ou J.T.; Wang F.R.; Lin D.L. Phys. Rev. E, 1997, 56, 2805. 